\title{
An Ugi Reaction Incorporating a Redox-Neutral Amine C-H Functionalization Step
}

\author{
Zhengbo Zhu and Daniel Seidel* \\ Department of Chemistry and Chemical Biology, Rutgers, The State University of New Jersey, \\ Piscataway, New Jersey 08854, United States
}

\section{Supporting Information}

General Information: Reagents and solvents were purchased from commercial sources and were purified by distillation or recrystallization prior to use unless stated otherwise. Cyclohexyl isocyanide was used as received. Benzyl isocyanide ${ }^{1}$ and isocyanobenzene ${ }^{2}$ were prepared following literature procedures. Purification of reaction products was carried out by flash column chromatography using Sorbent Technologies Standard Grade silica gel (60 ^, 230400 mesh). Analytical thin layer chromatography was performed on EM Reagent $0.25 \mathrm{~mm}$ silica gel $60 \quad \mathrm{~F}_{254}$ plates. Visualization was accomplished with UV light and Dragendorff-Munier stains, followed by heating. Melting points were recorded on a Thomas Hoover capillary melting point apparatus and are uncorrected. Infrared spectra were recorded on an ATI Mattson Genesis Series FT-Infrared spectrophotometer. Proton nuclear magnetic

resonance spectra $\left({ }^{1} \mathrm{H}-\mathrm{NMR}\right)$ were recorded on a Varian VNMRS-500 MHz. Chemical shifts are reported in ppm using the solvent as an internal standard $\left(\mathrm{CDCl}_{3}\right.$ at $\left.7.26 \mathrm{ppm}\right)$. Data are reported as app = apparent, $\mathrm{s}=$ singlet, $\mathrm{d}=$ doublet, $\mathrm{t}=$ triplet, $\mathrm{m}=$ multiplet, $\mathrm{comp}=$ complex, br = broad, coupling constant(s) in Hz. Proton-decoupled carbon nuclear magnetic resonance spectra $\left({ }^{13} \mathrm{C}\right.$-NMR) spectra were recorded on a Varian VNMRS-500 MHz. Chemical shifts are reported in ppm using the solvent as an internal standard $\left(\mathrm{CDCl}_{3}\right.$ at $\left.77.0 \mathrm{ppm}\right)$. Mass spectra were recorded on a Finnigan LCQ-DUO mass spectrometer. 
Table S1: Additional optimization data for 1,2,3,4-tetrahydroisoquinoline. ${ }^{\text {a }}$

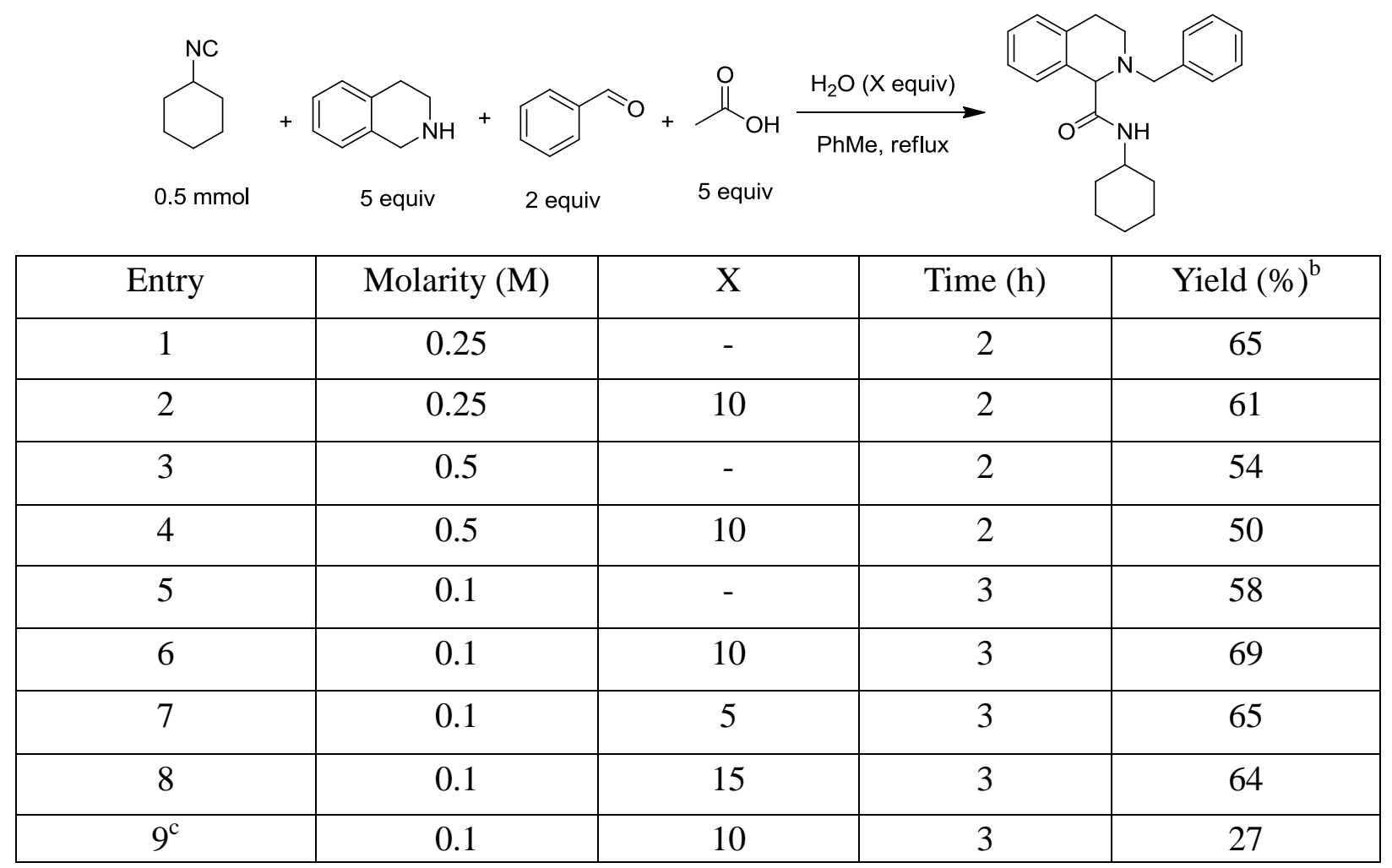

${ }^{a}$ Reactions were performed on a $0.5 \mathrm{mmol}$ scale. $\quad$ All substrates were mixed directly. ${ }^{\mathrm{b}}$. Isolated yields. ${ }^{\mathrm{c}}$.With 2 equiv of 1,2,3,4-tetrahydroisoquinoline.

\section{General procedure A for the redox-neutral $\alpha$-amidation:}

To a solution of $9 \mathrm{H}$-fluoren-9-one (180 mg, $1 \mathrm{mmol}, 2$ equiv) in toluene ( $2 \mathrm{~mL}, 0.25 \mathrm{M})$ was added the pyrrolidine $(0.21 \mathrm{~mL}, 2.5 \mathrm{mmol}, 5$ equiv), isocyanide ( $0.5 \mathrm{mmol}, 1$ equiv) and acetic acid $(0.14 \mathrm{~mL}, 2.5 \mathrm{mmol}, 5$ equiv). The resulting mixture was heated at reflux until the isocyanide was consumed as indicated by TLC. The reaction mixture was then allowed to cool to room temperature, diluted with $5 \mathrm{~mL}$ EtOAc, and washed with saturated aqueous $\mathrm{NaHCO}_{3}(3$ $\mathrm{x} 10 \mathrm{~mL})$. The combined aqueous layers were extracted with EtOAc $(3 \mathrm{x} 10 \mathrm{~mL})$. The combined organic layers were washed with brine $(20 \mathrm{~mL})$ and dried over anhydrous $\mathrm{Na}_{2} \mathrm{SO}_{4}$. The solvent was removed under reduced pressure and the residue purified by silica gel chromatography.

\section{General procedure B for the redox-neutral $\alpha$-amidation:}

To a solution of the aldehyde ( $1 \mathrm{mmol}, 2$ equiv) in toluene $(5 \mathrm{~mL}, 0.1 \mathrm{M})$ was added the amine ( $2.5 \mathrm{mmol}, 5$ equiv), isocyanide ( $0.5 \mathrm{mmol}, 1$ equiv), acetic acid $(0.14 \mathrm{~mL}, 2.5 \mathrm{mmol}, 5$ equiv) and distilled water $(90 \mu \mathrm{L}, 5 \mathrm{mmol}, 10$ equiv). The resulting mixture was heated at reflux until 
the isocyanide was consumed as indicated by TLC. The reaction mixture was then allowed to cool to room temperature, diluted with $5 \mathrm{~mL}$ EtOAc, and washed with saturated aqueous $\mathrm{NaHCO}_{3}(3 \times 10 \mathrm{~mL})$. The combined aqueous layers were extracted with EtOAc $(3 \times 10 \mathrm{~mL})$. The combined organic layers were washed with brine $(20 \mathrm{~mL})$ and dried over anhydrous $\mathrm{Na}_{2} \mathrm{SO}_{4}$. The solvent was removed under reduced pressure and the residue purified by silica gel chromatography.

$\mathrm{N}$-cyclohexyl-1-(9H-fluoren-9-yl)pyrrolidine-2-carboxamide 1a: Following the general

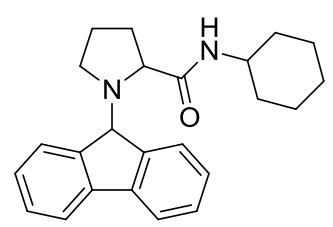
procedure A, cyclohexyl isocyanide, 9H-fluoren-9-one and pyrrolidine were heated at reflux for $18 \mathrm{~h}$. Product $1 \mathrm{a}$ was obtained as a yellow solid in $89 \%$ yield $(160 \mathrm{mg}),\left(\mathrm{R}_{\mathrm{f}}=0.28\right.$ in hexane/EtOAc $\left.80: 20 \mathrm{v} / \mathrm{v}\right) ; \mathrm{mp}=$ $113-115{ }^{\circ} \mathrm{C} ; \quad \operatorname{IR}(\mathrm{KBr}) 3310,2931,2848,1637,1522,1449,1302,1154$, 1127, 741, 731, 624; ${ }^{1} \mathrm{H} \mathrm{NMR}\left(\mathrm{CDCl}_{3}, 500 \mathrm{MHz}\right) \delta 7.65(\mathrm{~d}, J=8.8 \mathrm{~Hz}$, 1H), 7.58-7.55 (comp, 2H), 7.50-7.46 (m, 1H), 7.44-7.40 (m, 1H), 7.30-7.20 (comp, 3H), 7.17$7.12(\mathrm{~m}, 1 \mathrm{H}), 4.88(\mathrm{~s}, 1 \mathrm{H}), 3.89(\mathrm{dd}, J=9.7,3.7 \mathrm{~Hz}, 1 \mathrm{H}), 3.77-3.68(\mathrm{~m}, 1 \mathrm{H}), 2.60-2.55(\mathrm{~m}, 1 \mathrm{H})$, 2.25-2.18 (m, 1H), 2.12-2.02 (m, 1H), 1.93-1.83 (comp, 3H), 1.71-1.61 (comp, 2H), 1.59-1.47 (comp, 3H), 1.38-1.27 (comp, 2H), 1.24-1.10 (comp, 3H); $\left.{ }^{13} \mathrm{C} \mathrm{NMR} \mathrm{(125} \mathrm{MHz,} \mathrm{CDCl}_{3}\right) \delta$ 174.0, 144.8, 143.8, 141.6, 140.7, 128.62, 128.61, 127.7, 127.5, 126.0, 124.7, 120.3, 120.2, 66.0, $65.3,48.2,47.8,33.7,33.6,31.8,25.8,25.2,25.1,24.7 ; \quad m / z(E S I-M S) 361.0[\mathrm{M}+\mathrm{H}]^{+}$.

$N$-benzyl-1-(9H-fluoren-9-yl)pyrrolidine-2-carboxamide 1b: Following the general

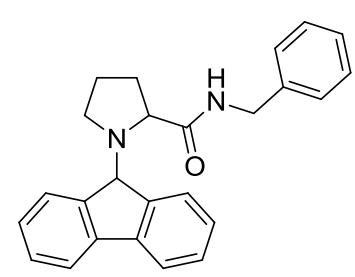
procedure A, benzyl isocyanide, $9 H$-fluoren-9-one and pyrrolidine were heated at reflux for $20 \mathrm{~h}$. Product $\mathbf{1 b}$ was obtained as a yellow solid in $65 \%$ yield $(119 \mathrm{mg}),\left(\mathrm{R}_{\mathrm{f}}=0.26\right.$ in hexane/EtOAc 80:20 v/v); $\mathrm{mp}=$ $119-120^{\circ} \mathrm{C}$; $\quad \operatorname{IR}(\mathrm{KBr}) 3351,3028,2933,2877,1649,1518,1450,1359$, 1298, 1201, 1126, 754, 733, 620, 478; ${ }^{1} \mathrm{H} \mathrm{NMR}\left(\mathrm{CDCl}_{3}, 500 \mathrm{MHz}\right) \delta$ 8.14-8.08 (m, 1H), 7.70-7.65 (comp, 2H), 7.58-7.54 (m, 1H), 7.41-7.16 (comp, 10H), $5.03(\mathrm{~s}, 1 \mathrm{H}), 4.59(\mathrm{dd}, J=14.7,6.2 \mathrm{~Hz}, 1 \mathrm{H}), 4.50(\mathrm{dd}, J=14.7,5.4 \mathrm{~Hz}, 1 \mathrm{H}), 4.08$ (dd, $J=9.8,3.5 \mathrm{~Hz}, 1 \mathrm{H}), 2.70-2.65(\mathrm{~m}, 1 \mathrm{H}), 2.40-2.33(\mathrm{~m}, 1 \mathrm{H}), 2.29-2.17(\mathrm{~m}, 1 \mathrm{H}), 2.10-2.03$ $(\mathrm{m}, 1 \mathrm{H}) ;{ }^{13} \mathrm{C}$ NMR $\left(125 \mathrm{MHz}, \mathrm{CDCl}_{3}\right) \delta 175.1,144.5,143.7,141.6,140.6,138.6,129.1,128.7$, 128.6, 127.9, 127.8, 127.6, 127.5, 125.8, 124.9, 120.3, 120.1, 66.1, 65.3, 48.3, 43.5, 31.8, 24.9; $\mathrm{m} / \mathrm{z}$ (ESI-MS) $369.4[\mathrm{M}+\mathrm{H}]^{+}$.

1-(9H-fluoren-9-yl)- $N$-phenylpyrrolidine-2-carboxamide 1c: Following the general

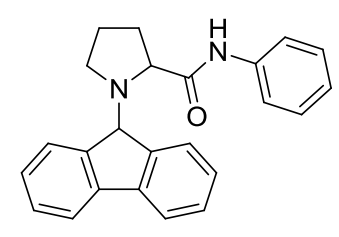
procedure $\mathrm{A}$, isocyanobenzene, $9 H$-fluoren-9-one and pyrrolidine were heated at reflux for $19 \mathrm{~h}$. Product 1c was obtained as a yellow solid in $29 \%$ yield $(52 \mathrm{mg}),\left(\mathrm{R}_{\mathrm{f}}=0.55\right.$ in hexane/EtOAc 80:20 v/v); $\mathrm{mp}=152-154{ }^{\circ} \mathrm{C}$; IR (KBr) 3284, 2964, 2839, 1681, 1601, 1518, 1442, 1311, 1258, 1080, $1025,744,500 ; \quad{ }^{1} \mathrm{H} \mathrm{NMR}\left(\mathrm{CDCl}_{3}, 500 \mathrm{MHz}\right) \delta 9.81(\mathrm{~s}, 1 \mathrm{H}), 7.65-7.60$ (comp, 3H), 7.59-7.54 (comp, 2H), 7.36-7.28 (comp, 5H), 7.22-7.16 (comp, 2H), 7.10-7.05 (m, 1H), 5.04 (s, 1H), 4.04 (dd, $J=9.8,3.4 \mathrm{~Hz}, 1 \mathrm{H}), 2.77-2.72(\mathrm{~m}, 1 \mathrm{H}), 2.39-2.32$ (m, 1H), 2.22- 
$2.13(\mathrm{~m}, 1 \mathrm{H}), 2.10-2.03(\mathrm{~m}, 1 \mathrm{H}), 1.67-1.61(\mathrm{comp}, 2 \mathrm{H}) ;{ }^{13} \mathrm{C} \mathrm{NMR}\left(125 \mathrm{MHz}, \mathrm{CDCl}_{3}\right) \delta 173.3$, 144.4, 143.4, 141.7, 140.8, 138.0, 129.4, 128.82, 128.81, 127.8, 127.6, 125.8, 124.8, 124.4, 120.4, $120.3,119.5,66.2,65.8,48.5,31.9,24.9 . ; \quad m / z$ (ESI-MS) $355.4[\mathrm{M}+\mathrm{H}]^{+}$.

$N$-cyclohexyl-1-(2,4,6-trimethylbenzyl)pyrrolidine-2-carboxamide 1d: Following the

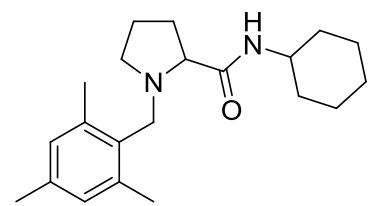
general procedure $\mathrm{B}$, cyclohexyl isocyanide, mesitaldehyde and pyrrolidine were heated at reflux for $12 \mathrm{~h}$. Product $1 \mathrm{~d}$ was obtained as a white solid in $68 \%$ yield $(112 \mathrm{mg}),\left(\mathrm{R}_{\mathrm{f}}=0.18\right.$ in hexane/EtOAc 80:20 $\mathrm{v} / \mathrm{v}) ; \quad \mathrm{mp}=113-115{ }^{\circ} \mathrm{C} ; \quad \mathrm{IR}(\mathrm{KBr}) 3324,2937,2851,2797,1648$, $1525,1450,1318,1173,1150,1113,885,849,668 ;{ }^{1} \mathrm{H} \mathrm{NMR}\left(\mathrm{CDCl}_{3}, 500 \mathrm{MHz}\right) \delta 6.90(\mathrm{~d}, J=$ $9.0 \mathrm{~Hz}, 1 \mathrm{H}), 6.81(\mathrm{~s}, 2 \mathrm{H}), 3.74(\mathrm{~d}, J=12.9 \mathrm{~Hz}, 1 \mathrm{H}), 3.67(\mathrm{~d}, J=12.9 \mathrm{~Hz}, 1 \mathrm{H}), 3.56-3.47(\mathrm{~m}$, $1 \mathrm{H}), 3.16(\mathrm{dd}, J=10.4,4.1 \mathrm{~Hz}, 1 \mathrm{H}), 2.99-2.93(\mathrm{~m}, 1 \mathrm{H}), 2.56-2.46(\mathrm{~m}, 1 \mathrm{H}), 2.36(\mathrm{~s}, 6 \mathrm{H}), 2.28-$ 2.18 (comp, 4H), 1.92-1.84 (m, 1H), 1.81-1.70 (comp, 2H), 1.68-1.52 (comp, 5H), 1.35-1.20 (comp, 2H), 1.15-0.90 (comp, 2H), 0.82-0.72 (m, 1H); $\left.{ }^{13} \mathrm{C} \mathrm{NMR} \mathrm{(125} \mathrm{MHz,} \mathrm{CDCl}_{3}\right) \delta 174.1$, 137.3, 136.8, 132.3, 129.5, 68.3, 54.9, 53.7, 47.6, 33.4, 32.7, 31.6, 25.8, 25.11, 25.10, 24.4, 21.0, 20.7; $\mathrm{m} / z$ (ESI-MS) $329.0[\mathrm{M}+\mathrm{H}]^{+}$.

$\mathrm{N}$-cyclohexyl-1-(2,6-dichlorobenzyl)pyrrolidine-2-carboxamide 1e: Following the general

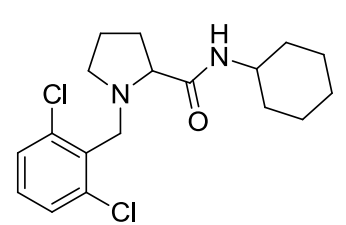
procedure B, cyclohexyl isocyanide, 2,6-dichlorobenzaldehyde and pyrrolidine were heated at reflux for $12 \mathrm{~h}$. Product 1e was obtained as a yellow solid in $60 \%$ yield $(106 \mathrm{mg}),\left(\mathrm{R}_{\mathrm{f}}=0.24\right.$ in hexane/EtOAc 80:20 v/v); $\mathrm{mp}=108-109{ }^{\circ} \mathrm{C} ; \quad \mathrm{IR}(\mathrm{KBr}) 3310,2934,2844,2364,1641,1521,1451$, $1293,1153,743,728,620 ; \quad{ }^{1} \mathrm{H}$ NMR $\left(\mathrm{CDCl}_{3}, 500 \mathrm{MHz}\right) \delta 7.31(\mathrm{~d}, J=8.2$ $\mathrm{Hz}, 1 \mathrm{H}), 7.26-7.22$ (comp, 2H), 7.10 (dd, $J=8.5,7.6 \mathrm{~Hz}, 1 \mathrm{H}), 4.00$ (d, $J=12.4 \mathrm{~Hz}, 1 \mathrm{H}), 3.92$ (d, $J=12.4 \mathrm{~Hz}, 1 \mathrm{H}), 3.55-3.45(\mathrm{~m}, 1 \mathrm{H}), 3.31(\mathrm{dd}, J=10.3,3.4 \mathrm{~Hz}, 1 \mathrm{H}), 3.03-2.96(\mathrm{~m}, 1 \mathrm{H}), 2.63$ (ddd, $J=10.8,9.0,5.8 \mathrm{~Hz}, 1 \mathrm{H}), 2.22-2.12(\mathrm{~m}, 1 \mathrm{H}), 1.93-1.86(\mathrm{~m}, 1 \mathrm{H}), 1.79-1.67$ (comp, $2 \mathrm{H})$, 1.66-1.49 (comp, 5H), 1.30-1.17 (comp, 2H), 1.12-0.97 (comp, 2H), 0.91-0.81 (m, 1H); ${ }^{13} \mathrm{C}$ NMR $\left(125 \mathrm{MHz}, \mathrm{CDCl}_{3}\right) \delta 173.7,136.6,134.2,129.3,128.8,66.9,54.7,54.0,47.6,33.5,32.9$, $\left.31.6,25.8,25.18,25.16,24.5 ; \quad \mathrm{m} / \mathrm{z}(\mathrm{ESI}-\mathrm{MS})\left({ }^{35} \mathrm{Cl}\right) 354.98[\mathrm{M}+\mathrm{H}]\right]^{+},\left({ }^{37} \mathrm{Cl}\right) 357.0[\mathrm{M}+\mathrm{H}]^{+}$.

$N$-cyclohexyl-2-(2,6-dichlorobenzyl)-1,2,3,4-tetrahydroisoquinoline-1-carboxamide 1f:

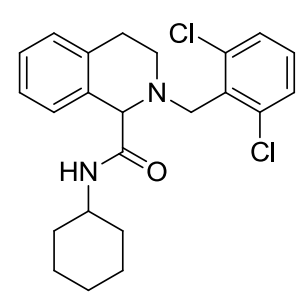

Following the general procedure B, cyclohexyl isocyanide, 2,6-dichlorobenzaldehyde and 1,2,3,4-tetrahydroisoquinoline were heated at reflux for $3 \mathrm{~h}$. Product $\mathbf{1 f}$ was obtained as a yellow solid in $68 \%$ yield $(142 \mathrm{mg}),\left(\mathrm{R}_{\mathrm{f}}=0.29\right.$ in hexane/EtOAc $\left.90: 10 \mathrm{v} / \mathrm{v}\right) ; \mathrm{mp}=65-66{ }^{\circ} \mathrm{C} ; \quad \mathrm{IR}$ (KBr) 3271, 2930, 2852, 1671, 1648, 1508, 1450, 1091, 745, 435; ${ }^{1} \mathrm{H}$ NMR $\left(\mathrm{CDCl}_{3}, 500 \mathrm{MHz}\right) \delta$ 7.54-7.48 (comp, 2H), 7.35-7.31 (comp, 2H), 7.21-7.14 (comp, 3H), 7.09-7.05 (m , 1H), $4.17(\mathrm{~s}, 1 \mathrm{H}), 4.13(\mathrm{~d}, J=12.7 \mathrm{~Hz}, 1 \mathrm{H}), 3.99$ (d, $J=12.6 \mathrm{~Hz}$, $1 \mathrm{H}$ ), 3.64-3.55 (m, 1H), 3.05 (ddd, $J=12.3,8.0,4.5 \mathrm{~Hz}, 1 \mathrm{H}$ ), 2.96 (ddd, $J=16.0,8.0,4.3 \mathrm{~Hz}$, $1 \mathrm{H}), 2.92-2.84(\mathrm{~m}, 1 \mathrm{H}), 2.67$ (app dt, $J=16.0,5.4 \mathrm{~Hz}, 1 \mathrm{H}$ ), 1.81-1.70 (comp, 2H), 1.68-1.50 (comp, 3H), 1.35-1.22 (comp, 2H), 1.19-1.01 (comp, 3H); $\left.{ }^{13} \mathrm{C} \mathrm{NMR} \mathrm{(125} \mathrm{MHz,} \mathrm{CDCl}_{3}\right) \delta$ 
170.9, 137.2, 134.2, 134.0, 132.2, 130.1, 129.6, 128.9, 128.4, 127.0, 126.0, 66.0, 53.4, 48.1, 46.3, $33.2,32.8,26.2,25.8,25.0,24.9 ; \quad \mathrm{m} / z(\mathrm{ESI}-\mathrm{MS})\left({ }^{35} \mathrm{Cl}\right) 417.72[\mathrm{M}+\mathrm{H}]^{+},\left({ }^{37} \mathrm{Cl}\right) 419.9[\mathrm{M}+$ $\mathrm{H}]^{+}$.

$N$-cyclohexyl-2-(2,4,6-trimethylbenzyl)-1,2,3,4-tetrahydroisoquinoline-1-carboxamide 1g:

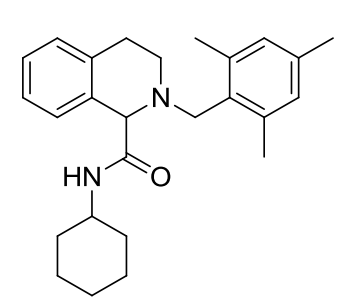

Following the general procedure B, cyclohexyl isocyanide, mesitaldehyde and 1,2,3,4-tetrahydroisoquinoline were heated at reflux for $3 \mathrm{~h}$. Product $1 \mathrm{~g}$ was obtained as a white solid in $64 \%$ yield $(124 \mathrm{mg}),\left(\mathrm{R}_{\mathrm{f}}=0.37\right.$ in hexane/EtOAc 90:10 v/v); $\mathrm{mp}=150-151^{\circ} \mathrm{C} ; \quad \mathrm{IR}(\mathrm{KBr}) 3322,2927$, 2849, 1644, 1521, 1450, 1319, 1231, 1136, 1103, 1073, 1031, 852, 745, 433; ${ }^{1} \mathrm{H}$ NMR $\left(\mathrm{CDCl}_{3}, 500 \mathrm{MHz}\right) \delta$ 7.48-7.45 (m, 1H), 7.22-7.16 (comp, 2H), 7.11-7.08 (m, 1H), 6.90-6.85 (comp,3H), $4.09(\mathrm{~s}, 1 \mathrm{H}), 3.95(\mathrm{~d}, J=12.6 \mathrm{~Hz}, 1 \mathrm{H}), 3.73-3.62$ (comp, 2H), 3.07-3.00 (m, 1H), 2.89-2.81 (m, 1H), 2.77-2.66 (comp, 2H), 2.33 (s, 6H), 2.29 (s, $3 \mathrm{H}), 1.91-1.83(\mathrm{~m}, 1 \mathrm{H}), 1.81-1.74(\mathrm{~m}, 1 \mathrm{H}), 1.72-1.55$ (comp, 3H), 1.41-1.25 (comp, 2H), 1.210.96 (comp, 3H); ${ }^{13} \mathrm{C}$ NMR $\left(125 \mathrm{MHz}, \mathrm{CDCl}_{3}\right) \delta 171.7,138.2,137.2,134.2,132.8,131.2$, 129.5, 128.9, 128.7, 127.0, 126.2, 67.8, 52.8, 48.2, 46.1, 33.1, 32.9, 27.2, 25.8, 25.0, 24.9, 21.1, 20.7; $\mathrm{m} / \mathrm{z}$ (ESI-MS) $391.2[\mathrm{M}+\mathrm{H}]^{+}$.

2-benzyl- $N$-cyclohexyl-1,2,3,4-tetrahydroisoquinoline-1-carboxamide 1h: Following the

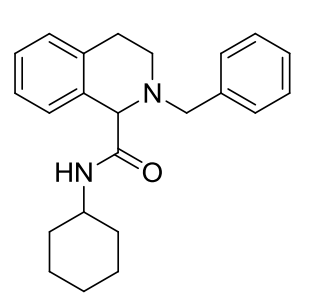
general procedure $\mathrm{B}$, cyclohexyl isocyanide, benzaldehyde and 1,2,3,4-tetrahydroisoquinoline were heated at reflux for $3 \mathrm{~h}$. Product $\mathbf{1 h}$ was obtained as a white solid in $69 \%$ yield $(120 \mathrm{mg}),\left(\mathrm{R}_{\mathrm{f}}=0.27\right.$ in hexane/EtOAc 90:10 v/v); $\mathrm{mp}=117-119^{\circ} \mathrm{C} ; \quad \mathrm{IR}(\mathrm{KBr}) 3346,3023,2936$, 2849, 2802, 1663, 1510, 1491, 1450, 1148, 753, 695, 607, 550; ${ }^{1} \mathrm{H}$ NMR $\left(\mathrm{CDCl}_{3}, 500 \mathrm{MHz}\right) \delta$ 7.61-7.56 (m, 1H), 7.40-7.28 (comp, 5H), 7.19-7.15 (comp, 2H), 7.12-7.06 (comp, 2H), $4.18(\mathrm{~s}, 1 \mathrm{H}), 3.95$ (d, $J=13.5 \mathrm{~Hz}, 1 \mathrm{H}), 3.79-3.69$ (m, 1H), 3.46 (d, $J=13.5 \mathrm{~Hz}, 1 \mathrm{H}$ ), 3.07 (ddd, $J=11.5,5.0,3.5 \mathrm{~Hz}, 1 \mathrm{H}$ ), 2.92 (ddd, $J=15.9,10.6,5.0 \mathrm{~Hz}$, $1 \mathrm{H}$ ), 2.70 (app dt, $J=16.1,3.5 \mathrm{~Hz}, 1 \mathrm{H}), 2.46$ (app td, $J=11.1,3.4 \mathrm{~Hz}, 1 \mathrm{H}), 1.94-1.87$ (m, 1H), 1.79-1.73 (m, 1H), 1.71-1.54 (comp, 3H), 1.40-1.26 (comp, 2H), 1.21-1.08 (comp, 2H), 1.06$0.97(\mathrm{~m}, 1 \mathrm{H}) ; \quad{ }^{13} \mathrm{C} \mathrm{NMR}\left(125 \mathrm{MHz}, \mathrm{CDCl}_{3}\right) \delta 172.1,138.1,134.1,132.8,128.9,128.8,128.7$, $127.6,127.5,127.0,126.4,68.7,60.3,48.0,46.8,33.2,33.1,29.0,25.8,25.0,24.9 ; \quad \mathrm{m} / z(\mathrm{ESI}-$ MS) $349.0[\mathrm{M}+\mathrm{H}]^{+}$.

2-(4-chlorobenzyl)- $N$-cyclohexyl-1,2,3,4-tetrahydroisoquinoline-1-carboxamide

1i:

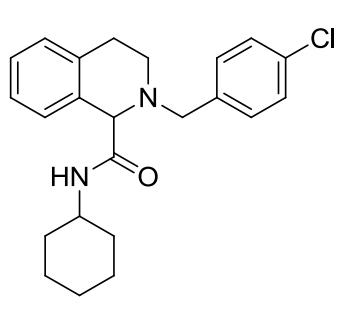

Following the general procedure B, cyclohexyl isocyanide, 4-chlorobenzaldehyde and 1,2,3,4-tetrahydroisoquinoline were heated at reflux for $3 \mathrm{~h}$. Product 1i was obtained as a white solid in $76 \%$ yield $(145 \mathrm{mg}),\left(\mathrm{R}_{\mathrm{f}}=0.27\right.$ in hexane/EtOAc $\left.90: 10 \mathrm{v} / \mathrm{v}\right) ; \mathrm{mp}=103-105^{\circ} \mathrm{C}$; IR (KBr) 3264, 3070, 2930, 2851, 1648, 1635, 1552, 1490, 1449, 1090, $1014,741,485 ;{ }^{1} \mathrm{H}$ NMR $\left(\mathrm{CDCl}_{3}, 500 \mathrm{MHz}\right) \delta 7.57-7.52(\mathrm{~m}, 1 \mathrm{H})$, 7.35-7.31 (comp, 2H), 7.29-7.24 (comp, 2H), 7.19-7.13 (comp, 2H), 7.10-7.05 (m, 1H), 6.95 (d, 
$\mathrm{J}=8.6 \mathrm{~Hz}, 1 \mathrm{H}), 4.16(\mathrm{~s}, 1 \mathrm{H}), 3.89(\mathrm{~d}, J=13.7 \mathrm{~Hz}, 1 \mathrm{H}), 3.79-3.69(\mathrm{~m}, 1 \mathrm{H}), 3.44(\mathrm{~d}, J=13.7 \mathrm{~Hz}$, 1H), 3.06-3.00 (m, 1H), 2.91 (ddd, $J=15.9,10.6,5.0 \mathrm{~Hz}, 1 \mathrm{H}), 2.70$ (app dt, $J=16.2,3.5 \mathrm{~Hz}$, $1 \mathrm{H}), 2.44(\mathrm{app} t \mathrm{td}, J=11.0,3.5 \mathrm{~Hz}, 1 \mathrm{H}), 1.91-1.84(\mathrm{~m}, 1 \mathrm{H}), 1.78-1.71(\mathrm{~m}, 1 \mathrm{H}), 1.70-1.53$ (comp, 3H), 1.39-1.24 (comp, 2H), 1.18-1.07 (comp, 2H), 1.04-0.94 (m, 1H); ${ }^{13} \mathrm{C}$ NMR (125 $\left.\mathrm{MHz} \mathrm{CDCl}_{3}\right) \delta 172.0,136.5,133.9,133.4,132.6,130.2,128.9,128.8,127.4,127.1,126.4,68.7$, $59.5,48.0,46.8,33.3,33.1,29.0,25.7,25.0,24.9 ; \quad \mathrm{m} / \mathrm{z}(\mathrm{ESI}-\mathrm{MS})\left({ }^{35} \mathrm{Cl}\right) 383.14[\mathrm{M}+\mathrm{H}]+\left({ }^{37} \mathrm{Cl}\right)$ $385.1[\mathrm{M}+\mathrm{H}]^{+}$.

2-(4-cyanobenzyl)- $N$-cyclohexyl-1,2,3,4-tetrahydroisoquinoline-1-carboxamide

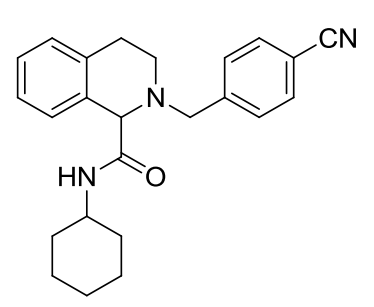

Following the general procedure $\mathrm{B}$, cyclohexyl isocyanide, 4-formylbenzonitrile and 1,2,3,4-tetrahydroisoquinoline were heated at reflux for $3 \mathrm{~h}$. Product $\mathbf{1 j}$ was obtained as a yellow oil in $67 \%$ yield (125 $\mathrm{mg}),\left(\mathrm{R}_{\mathrm{f}}=0.10\right.$ in hexane/EtOAc 90:10 v/v); IR $(\mathrm{KBr}) 3373,2931$, 2854, 2228, 1656, 1510, 1451, 1386, 1311, 1151, 1093, 890, 815, 736;

${ }^{1} \mathrm{H} \mathrm{NMR}\left(\mathrm{CDCl}_{3}, 500 \mathrm{MHz}\right) \delta 7.69-7.63$ (comp, 2H), 7.52-7.48 (m, 1H), 7.47-7.43 (comp, 2H), 7.20-7.13 (comp , 2H), 7.11-7.05 (m, 1H), 6.78 (d, J=8.6 Hz, 1H) 4.17 (s, 1H), $3.96(\mathrm{~d}, J=14.4 \mathrm{~Hz}, 1 \mathrm{H}), 3.76-3.66(\mathrm{~m}, 1 \mathrm{H}), 3.55(\mathrm{~d}, J=14.4 \mathrm{~Hz}, 1 \mathrm{H}), 3.02-2.90$ (comp, 2H), 2.72 (app dt, $J=16.2,3.0 \mathrm{~Hz}, 1 \mathrm{H}), 2.48(\mathrm{~m}, 1 \mathrm{H}), 1.85-1.78(\mathrm{~m}, 1 \mathrm{H}), 1.77-1.71$ (m, 1H), 1.68-1.52 (comp, 3H), 1.38-1.23 (comp, 2H), 1.16-1.03 (comp, 2H), 1.02-0.92 (m, 1H); ${ }^{13} \mathrm{C}$ NMR $\left(125 \mathrm{MHz}, \mathrm{CDCl}_{3}\right) \delta 171.7,143.7,133.6,132.6,132.4,129.3,128.8,127.3,127.2$, $126.6,118.9,111.6,68.9,59.7,48.0,47.2,33.3,33.0,28.9,25.7,25.0,24.8 ; \quad \mathrm{m} / z$ (ESI-MS) $374.6[\mathrm{M}+\mathrm{H}]^{+}$.

$N$-cyclohexyl-2-(4-methoxybenzyl)-1,2,3,4-tetrahydroisoquinoline-1-carboxamide

1k:

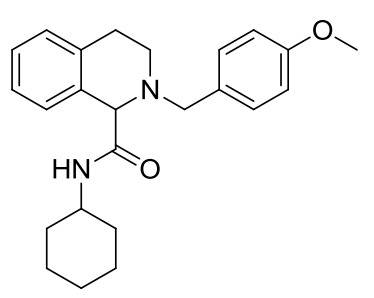

Following the general procedure B, cyclohexyl isocyanide, 4-methoxybenzaldehyde and 1,2,3,4-tetrahydroisoquinoline were heated at reflux for $3 \mathrm{~h}$. Product $1 \mathrm{k}$ was obtained as a white solid in $68 \%$ yield $(130 \mathrm{mg}),\left(\mathrm{R}_{\mathrm{f}}=0.20\right.$ in hexane/EtOAc $\left.90: 10 \mathrm{v} / \mathrm{v}\right) ; \mathrm{mp}=133-135{ }^{\circ} \mathrm{C}$; IR (KBr) 3368, 2931, 2856, 2834, 2354, 1670, 1611, 1508, 1443, 1247, $1173,1080,1032,813,752,525 ;{ }^{1} \mathrm{H} \mathrm{NMR}\left(\mathrm{CDCl}_{3}, 500 \mathrm{MHz}\right) \delta 7.60$ 7.55 (m, 1H), 7.26-7.22 (comp, 2H), 7.18-7.14 (comp, 2H), 7.13-7.05 (comp, 2H), 6.92-6.88 (comp, 2H), 4.15 (s, 1H), $3.88(\mathrm{~d}, J=13.3 \mathrm{~Hz}, 1 \mathrm{H}), 3.82(\mathrm{~s}, 3 \mathrm{H}), 3.78-3.70(\mathrm{~m}, 1 \mathrm{H}), 3.40(\mathrm{~d}, J=$ $13.3 \mathrm{~Hz}, 1 \mathrm{H}$ ), 3.06 (ddd, $J=11.5,5.0,3.6 \mathrm{~Hz}, 1 \mathrm{H}$ ), 2.89 (ddd, $J=16.2,10.5,5.0 \mathrm{~Hz}, 1 \mathrm{H}$ ), 2.69 (app dt, $J=16.1,3.5 \mathrm{~Hz}, 1 \mathrm{H}), 2.43(\operatorname{app~td}, J=11.1,3.4 \mathrm{~Hz}, 1 \mathrm{H}), 1.96-1.89(\mathrm{~m}, 1 \mathrm{H}), 1.79-1.54$ (comp, 4H), 1.41-1.26 (comp, 2H), 1.22-1.09 (comp, 2H), 1.06-0.96 (m, 1H); ${ }^{13} \mathrm{C}$ NMR (125 $\left.\mathrm{MHz}, \mathrm{CDCl}_{3}\right) \delta 172.2,159.2,134.2,132.8,130.2,129.9,128.7,127.5,127.0,126.4,114.1,68.5$, 59.6, 55.5, 48.0, 46.6, 33.3, 33.1, 29.0, 25.8, 25.1, 24.9; $\mathrm{m} / z(\mathrm{ESI}-\mathrm{MS}) 379.0[\mathrm{M}+\mathrm{H}]^{+}$. $\mathrm{X}$-ray quality crystals of $\mathbf{1 k}$ were obtained from hexane/dichloromethane through slow diffusion at room temperature. 


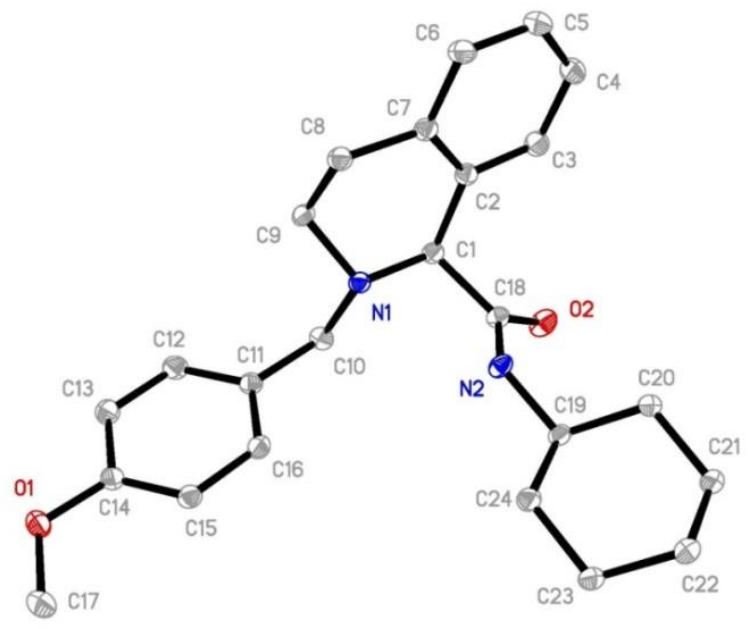

The requisite CIF has been submitted to the journal and deposited with the CCDC (deposition \# 1441887).

$N$-cyclohexyl-2-(3-methoxybenzyl)-1,2,3,4-tetrahydroisoquinoline-1-carboxamide 11:<smiles>COc1cccc(CN2CCc3ccccc3C2C(=O)NC2CCCCC2)c1</smiles>
Following the general procedure B, cyclohexyl isocyanide, 3-methoxybenzaldehyde and 1,2,3,4-tetrahydroisoquinoline were heated at reflux for $3 \mathrm{~h}$. Product 11 was obtained as a white solid in 74\% yield $(140 \mathrm{mg}),\left(\mathrm{R}_{\mathrm{f}}=0.19\right.$ in hexane/EtOAc $\left.90: 10 \mathrm{v} / \mathrm{v}\right) ; \quad \mathrm{mp}=90$ $92{ }^{\circ} \mathrm{C}$; IR (KBr) 3310, 3052, 3019, 2933, 2849, 1648, 1522, 1487, $1449,1277,1153,1049,778,750,688,568,435 ;{ }^{1} \mathrm{H}$ NMR $\left(\mathrm{CDCl}_{3}\right.$, $500 \mathrm{MHz}) \delta$ 7.60-7.56 (m, 1H), 7.31-7.25 (m, 1H), 7.18-7.06 (comp, 4H), 6.95-6.88 (comp, 2H), 6.86-6.82 (m, 1H), $4.17(\mathrm{~s}, 1 \mathrm{H}), 3.92(\mathrm{~d}, J=13.6 \mathrm{~Hz}, 1 \mathrm{H}), 3.81(\mathrm{~s}, 3 \mathrm{H}), 3.78-3.69(\mathrm{~m}, 1 \mathrm{H})$, $3.42(\mathrm{~d}, J=13.6 \mathrm{~Hz}, 1 \mathrm{H}), 3.07(\mathrm{ddd}, J=11.5,5.0,3.5 \mathrm{~Hz}, 1 \mathrm{H}), 2.92(\mathrm{ddd}, J=15.9,10.7,5.0 \mathrm{~Hz}$, 1H), 2.69 (app dt, $J=16.2,3.5 \mathrm{~Hz}, 1 \mathrm{H}), 2.44$ (app td, $J=11.1,3.4 \mathrm{~Hz}, 1 \mathrm{H}), 1.93-1.87$ (m, 1H), 1.79-1.72 (m, 1H), 1.70-1.53 (comp, 3H), 1.39-1.25 (comp, 2H), 1.20-1.07 (comp, 2H), 1.05$0.96(\mathrm{~m}, 1 \mathrm{H}) ;{ }^{13} \mathrm{C} \mathrm{NMR}\left(125 \mathrm{MHz}, \mathrm{CDCl}_{3}\right) \delta 172.2,160.0,139.7,134.1,132.7,129.8,128.8$, 127.4, 127.0, 126.4, 121.2, 114.6, 112.8, 68.7, 60.2, 55.4, 48.0, 46.8, 33.2, 33.1, 29.0, 25.8, 25.1, $24.9 ; \quad m / z\left(\right.$ ESI-MS) $379.4[\mathrm{M}+\mathrm{H}]^{+}$.

2-(3-bromobenzyl)- $N$-cyclohexyl-1,2,3,4-tetrahydroisoquinoline-1-carboxamide 1m:<smiles>O=C(NC1CCCCC1)C1c2ccccc2CCN1Cc1cccc(Br)c1</smiles>

Following the general procedure B, cyclohexyl isocyanide, 3-bromobenzaldehyde and 1,2,3,4-tetrahydroisoquinoline were heated at reflux for $3 \mathrm{~h}$. Product $\mathbf{1 m}$ was obtained as a yellow solid in $74 \%$ yield $(158 \mathrm{mg}),\left(\mathrm{R}_{\mathrm{f}}=0.21\right.$ in hexane/EtOAc $\left.90: 10 \mathrm{v} / \mathrm{v}\right) ; \quad \mathrm{mp}=83-85^{\circ} \mathrm{C} ; \quad$ IR (KBr) 3262, 3063, 2928, 2851, 1648, 1560, 1445, 1244, 1088, 925, 882, 777, 740, 669, 425; ${ }^{1} \mathrm{H} \mathrm{NMR}\left(\mathrm{CDCl}_{3}, 500 \mathrm{MHz}\right) \delta 7.57-7.53(\mathrm{~m}, 1 \mathrm{H})$, 
7.52-7.50 (m, 1H), 7.42 (app dt, $J=7.3,1.9 \mathrm{~Hz}, 1 \mathrm{H}), 7.26-7.20$ (comp, 2H), 7.18-7.14 (comp, 2H), 7.10-7.06 (m, 1H), 6.97 (d, J = 8.6 Hz, 1H), $4.16(\mathrm{~s}, 1 \mathrm{H}), 3.89$ (d, J = $13.7 \mathrm{~Hz}, 1 \mathrm{H}), 3.78-$ $3.69(\mathrm{~m}, 1 \mathrm{H}), 3.44$ (d, $J=13.8 \mathrm{~Hz}, 1 \mathrm{H}), 3.04$ (ddd, $J=11.5,5.1,3.7 \mathrm{~Hz}, 1 \mathrm{H}), 2.92$ (ddd, $J=15.8$, $10.5,5.0 \mathrm{~Hz}, 1 \mathrm{H}), 2.71$ (app dt, $J=16.2,3.6 \mathrm{~Hz}, 1 \mathrm{H}), 2.47$ (ddd, $J=11.4,10.5,3.5 \mathrm{~Hz}, 1 \mathrm{H}$ ), 1.91-1.85 (m, 1H), 1.78-1.71 (m, 1H), 1.70-1.52 (comp, 3H), 1.40-1.25 (comp, 2H), 1.21-1.09 (comp, 2H), 1.06-0.96 (m, 1H); ${ }^{13} \mathrm{C}$ NMR (125 MHz, $\left.\mathrm{CDCl}_{3}\right) \delta 171.9,140.6,133.9,132.5$, 131.8, 130.8, 130.3, 128.8, 127.49, 127.46, 127.1, 126.4, 123.0, 68.7, 59.6, 48.0, 47.0, 33.2, 33.0, $28.9,25.7,25.0,24.8 ; \quad \mathrm{m} / z(\mathrm{ESI}-\mathrm{MS})\left({ }^{79} \mathrm{Br}\right) 427.13[\mathrm{M}+\mathrm{H}]^{+},\left({ }^{81} \mathrm{Br}\right) 429.1[\mathrm{M}+\mathrm{H}]^{+}$.

$N$-cyclohexyl-2-(3-methylbenzyl)-1,2,3,4-tetrahydroisoquinoline-1-carboxamide 1n:

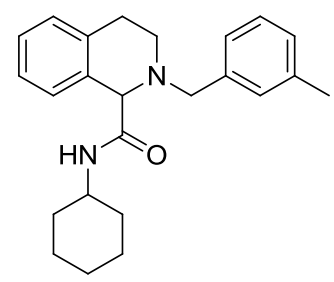

Following the general procedure B, cyclohexyl isocyanide, 3-methylbenzaldehyde and 1,2,3,4-tetrahydroisoquinoline were heated at reflux for $3 \mathrm{~h}$. Product $1 \mathrm{n}$ was obtained as a white solid in $72 \%$ yield $(130 \mathrm{mg}),\left(\mathrm{R}_{\mathrm{f}}=0.29\right.$ in hexane/EtOAc $\left.90: 10 \mathrm{v} / \mathrm{v}\right) ; \quad \mathrm{mp}=103-104{ }^{\circ} \mathrm{C}$; IR (KBr) 3375, 2927, 2856, 1668, 1506, 1452, 1365, 1139, 1083, 789, 755, 748, 701, 571, 538, 440; ${ }^{1} \mathrm{H} \mathrm{NMR}\left(\mathrm{CDCl}_{3}, 500 \mathrm{MHz}\right) \delta 7.52-7.48(\mathrm{~m}$, 1H), 7.19-7.14 (m, 1H), 7.11-6.97 (comp, 7H), 4.08 (s, 1H), 3.82 (d, J=13.4 Hz, 1H), 3.70$3.61(\mathrm{~m}, 1 \mathrm{H}), 3.32(\mathrm{~d}, J=13.4 \mathrm{~Hz}, 1 \mathrm{H}), 2.98(\mathrm{ddd}, J=11.5,5.0,3.6 \mathrm{~Hz}, 1 \mathrm{H}), 2.82(\mathrm{ddd}, J=15.8$, 10.6, $4.9 \mathrm{~Hz}, 1 \mathrm{H}), 2.61$ (app dt, $J=16.1,3.5 \mathrm{~Hz}, 1 \mathrm{H}), 2.35(\mathrm{app} t \mathrm{t}, J=11.1,3.4 \mathrm{~Hz}, 1 \mathrm{H}), 2.28$ (s, $3 \mathrm{H}), 1.87-1.80$ (m, 1H), 1.71-1.44 (comp, 4H), 1.32-1.17 (comp, 2H), 1.14-1.00 (comp, 2H), $0.98-0.88(\mathrm{~m}, 1 \mathrm{H}) ;{ }^{13} \mathrm{C} \mathrm{NMR}\left(125 \mathrm{MHz}, \mathrm{CDCl}_{3}\right) \delta 172.2,138.4,138.0,134.2,132.8,129.8$, $128.74,128.67,128.4,127.5,127.0,126.4,126.0,68.6,60.3,48.0,46.7,33.2,33.1,29.0,25.8$, 25.0, 24.9, 21.8; $m / z(\mathrm{ESI}-\mathrm{MS}) 363.2[\mathrm{M}+\mathrm{H}]^{+}$.

$N$-cyclohexyl-2-(2-methylbenzyl)-1,2,3,4-tetrahydroisoquinoline-1-carboxamide 1o:

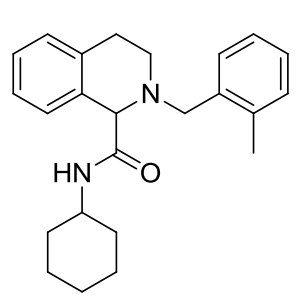

Following the general procedure $\mathrm{B}$, cyclohexyl isocyanide, 2-methylbenzaldehyde and 1,2,3,4-tetrahydroisoquinoline were heated at reflux for $3 \mathrm{~h}$. Product 10 was obtained as a white solid in 64\% yield (115 $\mathrm{mg}),\left(\mathrm{R}_{\mathrm{f}}=0.34\right.$ in hexane/EtOAc 90:10 v/v); $\mathrm{mp}=101-102{ }^{\circ} \mathrm{C} ; \quad \mathrm{IR}(\mathrm{KBr})$ 3273, 3065, 2931, 1635, 1551, 1496, 1451, 1229, 1085, 887, 741, 684; ${ }^{1} \mathrm{H}$ NMR $\left(\mathrm{CDCl}_{3}, 500 \mathrm{MHz}\right) \delta 7.50-7.46(\mathrm{~m}, 1 \mathrm{H}), 7.29-7.25(\mathrm{~m}, 1 \mathrm{H}), 7.14-7.06$ (comp, 5H), 7.02-6.99 (m, 1H), $6.91(\mathrm{~d}, J=8.5 \mathrm{~Hz}, 1 \mathrm{H}), 4.11(\mathrm{~s}, 1 \mathrm{H}), 3.73(\mathrm{~d}, J=14.0 \mathrm{~Hz}, 1 \mathrm{H})$, $3.63-3.55(\mathrm{~m}, 1 \mathrm{H}), 3.50(\mathrm{~d}, J=14.0 \mathrm{~Hz}, 1 \mathrm{H}), 2.97$ (app dt, J = 11.7, $4.7 \mathrm{~Hz}, 1 \mathrm{H}), 2.83$ (ddd, J = 15.3, 9.8, 5.0 Hz, 1H), 2.66 (app dt, $J=16.2,4.1 \mathrm{~Hz}, 1 \mathrm{H}), 2.43$ (ddd, J = 11.7, 9.8, 3.7 Hz, 1H), 2.26 (s, 3H), 1.71-1.61 (comp, 2H), 1.54-1.40 (comp, 3H), 1.25-1.14 (comp, 2H), 1.05-0.83 (comp, 3H); $\left.{ }^{13} \mathrm{C} \mathrm{NMR} \mathrm{(125} \mathrm{MHz,} \mathrm{CDCl}_{3}\right) \delta 172.0,136.9,136.3,134.1,132.8,130.7,128.7$, 128.6, 127.9, 127.5, 127.0, 126.4, 126.3, 69.0, 57.6, 48.0, 47.0, 33.0, 32.9, 28.5, 25.7, 24.9, 24.83, 19.7; $\mathrm{m} / z(\mathrm{ESI}-\mathrm{MS}) 363.2[\mathrm{M}+\mathrm{H}]^{+}$. 
$N$-cyclohexyl-2-(2-fluorobenzyl)-1,2,3,4-tetrahydroisoquinoline-1-carboxamide 1p:

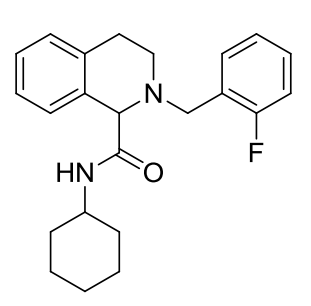

Following the general procedure B, cyclohexyl isocyanide, 2-fluorobenzaldehyde and 1,2,3,4-tetrahydroisoquinoline were heated at reflux for $3 \mathrm{~h}$. Product $\mathbf{1 p}$ was obtained as a yellow oil in $82 \%$ yield (150 $\mathrm{mg}),\left(\mathrm{R}_{\mathrm{f}}=0.39\right.$ in hexane/EtOAc 90:10 v/v); IR (KBr) 3374, 3064, 2931, 2854, 2359, 1671, 1510, 1492, 1453, 1368, 1229, 1098, 1085, 1033, 890, 757; ${ }^{1} \mathrm{H} \mathrm{NMR}\left(\mathrm{CDCl}_{3}, 500 \mathrm{MHz}\right) \delta$ 7.64-7.59 (m, 1H), 7.36-7.27 (comp, 2H), 7.22-7.18 (m, 1H), 7.17-7.11 (comp, 3H), 7.10-7.03 (comp, 2H), 4.17 (s, 1H), 3.97 (d, J = 13.3 Hz, 1H), 3.76-3.66 (m, 1H), $3.54(\mathrm{~d}, J=13.3 \mathrm{~Hz}, 1 \mathrm{H}), 3.03$ (ddd, $J=11.4,4.9,3.5 \mathrm{~Hz}, 1 \mathrm{H}), 2.90$ (ddd, $J=15.9,10.8,5.0 \mathrm{~Hz}, 1 \mathrm{H}), 2.68$ (app dt, $J=16.1,3.4 \mathrm{~Hz}, 1 \mathrm{H}), 2.46$ (app td, $J=11.1,3.3$ $\mathrm{Hz}, 1 \mathrm{H}$ ), 1.97-1.89 (m, 1H), 1.76-1.53 (comp, 4H), 1.39-1.23 (comp, 2H), 1.21-1.07 (comp, 2H), $1.06-0.97(\mathrm{~m}, 1 \mathrm{H}) ;{ }^{13} \mathrm{C} \mathrm{NMR}\left(125 \mathrm{MHz}, \mathrm{CDCl}_{3}\right) \delta 171.9,161.9\left(\mathrm{~d}, J_{\mathrm{C}-\mathrm{F}}=247.1 \mathrm{~Hz}\right)$, 134.1, 132.8, $131.8\left(\mathrm{~d}, J_{\mathrm{C}-\mathrm{F}}=4.6 \mathrm{~Hz}\right), 129.6\left(\mathrm{~d}, J_{\mathrm{C}-\mathrm{F}}=8.3 \mathrm{~Hz}\right), 128.6,127.5,127.0,126.4,124.7$ $\left(\mathrm{d}, J_{\mathrm{C}-\mathrm{F}}=14.4 \mathrm{~Hz}\right), 124.3\left(\mathrm{~d}, J_{\mathrm{C}-\mathrm{F}}=3.5 \mathrm{~Hz}\right), 115.9\left(\mathrm{~d}, J_{\mathrm{C}-\mathrm{F}}=22.0 \mathrm{~Hz}\right), 68.8,54.4,48.2,46.8,33.0$, $32.9,29.0,25.8,25.1,25.0 ; \quad \mathrm{m} / z$ (ESI-MS) $367.2[\mathrm{M}+\mathrm{H}]^{+}$.

2-(2-chlorobenzyl)- $N$-cyclohexyl-1,2,3,4-tetrahydroisoquinoline-1-carboxamide 1q:

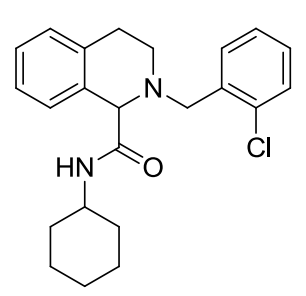
Following the general procedure B, cyclohexyl isocyanide, 2-chlorobenzaldehyde and 1,2,3,4-tetrahydroisoquinoline were heated at reflux for $3 \mathrm{~h}$. Product $\mathbf{1 q}$ was obtained as a white solid in $75 \%$ yield (143 $\mathrm{mg}),\left(\mathrm{R}_{\mathrm{f}}=0.24\right.$ in hexane/EtOAc $\left.90: 10 \mathrm{v} / \mathrm{v}\right) ; \mathrm{mp}=117-118^{\circ} \mathrm{C} ; \quad \mathrm{IR}(\mathrm{KBr})$ 3289, 2930, 2853, 1636, 1549, 1442, 1230, 1035, 748, 696; ${ }^{1} \mathrm{H}$ NMR $\left(\mathrm{CDCl}_{3}, 500 \mathrm{MHz}\right) \delta 7.60-7.56(\mathrm{~m}, 1 \mathrm{H}), 7.43-7.37$ (comp, 2H), 7.30-7.22 (comp, 2H), 7.21-7.15 (comp, 3H), 7.12-7.07 (m, 1H), $4.21(\mathrm{~s}, 1 \mathrm{H}), 3.87$ (d, J = 14.1 Hz, 1H), $3.81(\mathrm{~d}, J=14.1 \mathrm{~Hz}, 1 \mathrm{H}), 3.70-3.61(\mathrm{~m}, 1 \mathrm{H}), 3.07$ (app dt, $J=11.9,5.0 \mathrm{~Hz}, 1 \mathrm{H}), 2.90$ (ddd, $J=$ 14.7, 9.3, 4.9 Hz, 1H), 2.79 (app dt, $J=16.3,4.4 \mathrm{~Hz}, 1 \mathrm{H}$ ) , 2.63 (ddd, $J=11.8,9.3,3.8 \mathrm{~Hz}, 1 \mathrm{H}$ ), 1.79-1.70 (comp, 2H), 1.64-1.49 (comp, 3H), 1.34-1.22 (comp, 2H), 1.15-0.95 (comp, 3H); ${ }^{13} \mathrm{C}$ NMR $\left(125 \mathrm{MHz}, \mathrm{CDCl}_{3}\right) \delta 171.6,135.8,134.7,134.0,132.6,130.5,130.1,129.0,128.7$, $128.3,127.13,127.06,126.3,68.0,57.3,48.0,47.1,33.0,32.9,28.1,25.8,24.94,24.86 ; \mathrm{m} / \mathrm{z}$ (ESI-MS) $\left({ }^{35} \mathrm{Cl}\right) 383.14[\mathrm{M}+\mathrm{H}]^{+},\left({ }^{37} \mathrm{Cl}\right) 385.2[\mathrm{M}+\mathrm{H}]^{+}$.

$N$-cyclohexyl-2-(naphthalen-2-ylmethyl)-1,2,3,4-tetrahydroisoquinoline-1-carboxamide 1r:

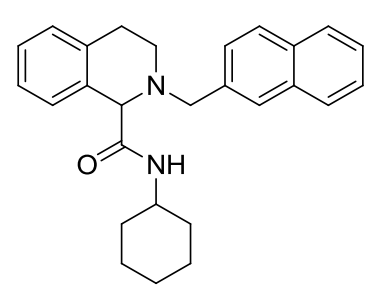

Following the general procedure B, cyclohexyl isocyanide, 2-naphthaldehyde and 1,2,3,4-tetrahydroisoquinoline were heated at reflux for $3 \mathrm{~h}$. Product $1 \mathbf{r}$ was obtained as a white solid in $71 \%$ yield $(141 \mathrm{mg}),\left(\mathrm{R}_{\mathrm{f}}=0.26\right.$ in hexane/EtOAc $\left.90: 10 \mathrm{v} / \mathrm{v}\right) ; \quad \mathrm{mp}=150-151^{\circ} \mathrm{C}$; IR (KBr) 3379, 3023, 2923, 1668, 1504, 1451, 857, 816, 759, 480; ${ }^{1} \mathrm{H}$ $\mathrm{NMR}\left(\mathrm{CDCl}_{3}, 500 \mathrm{MHz}\right) \delta 7.90-7.83$ (comp, 3H), 7.80-7.78 (m, 1H), 7.65-7.61 (m, 1H), 7.55-7.47 (comp, 3H), 7.23-7.16 (comp, 3H), 7.13-7.08 (m, 1H), 4.7 (s, 1H), $4.13(\mathrm{~d}, J=13.4 \mathrm{~Hz}, 1 \mathrm{H}), 3.85-3.75(\mathrm{~m}, 1 \mathrm{H}), 3.64(\mathrm{~d}, J=13.5 \mathrm{~Hz}, 1 \mathrm{H}), 3.14$ (ddd, $J=11.6,5.0$, $3.7 \mathrm{~Hz}, 1 \mathrm{H}), 2.94$ (ddd, $J=15.8,10.5,5.0 \mathrm{~Hz}, 1 \mathrm{H}), 2.73$ (app dt, $J=16.2,3.6 \mathrm{~Hz}, 1 \mathrm{H}), 2.56-$ 
$2.49(\mathrm{~m}, 1 \mathrm{H}), 1.99-1.92(\mathrm{~m}, 1 \mathrm{H}), 1.84-1.77(\mathrm{~m}, 1 \mathrm{H}), 1.74-1.56$ (comp, 3H), 1.43-1.03 (comp, $5 \mathrm{H}) ;{ }^{13} \mathrm{C}$ NMR $\left(125 \mathrm{MHz}, \mathrm{CDCl}_{3}\right) \delta 172.1,135.7,134.1,133.6,133.1,132.8,128.8,128.6$, 127.96, 127.95, 127.8, 127.6, 127.1, 126.8, 126.5, 126.4, 126.2, 68.8, 60.4, 48.0, 46.9, 33.3, 33.2, 29.0, 25.8, 25.0, 24.9; $\mathrm{m} / \mathrm{z}$ (ESI-MS) $399.4[\mathrm{M}+\mathrm{H}]^{+}$.

$N$-cyclohexyl-2-(thiophen-2-ylmethyl)-1,2,3,4-tetrahydroisoquinoline-1-carboxamide 1 s:

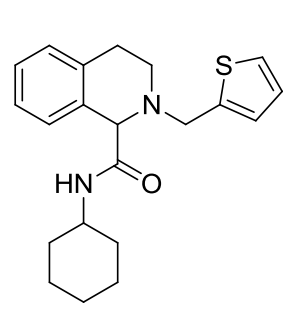

Following the general procedure B, cyclohexyl isocyanide, thiophene-2-carbaldehyde and 1,2,3,4-tetrahydroisoquinoline were heated at reflux for $3 \mathrm{~h}$. Product $1 \mathrm{~s}$ was obtained as a yellow solid in $76 \%$ yield (135 $\mathrm{mg}),\left(\mathrm{R}_{\mathrm{f}}=0.24\right.$ in hexane/EtOAc $\left.90: 10 \mathrm{v} / \mathrm{v}\right) ; \quad \mathrm{mp}=100-102{ }^{\circ} \mathrm{C} ; \quad \mathrm{IR}(\mathrm{KBr})$ 3372, 2932, 2856, 1670, 1507, 1452, 1369, 1140, 754, 733, 709; ${ }^{1} \mathrm{H}$ NMR $\left(\mathrm{CDCl}_{3}, 500 \mathrm{MHz}\right) \delta 7.61-7.56(\mathrm{~m}, 1 \mathrm{H}), 7.28-7.25(\mathrm{~m}, 1 \mathrm{H}), 7.19-7.05$ (comp, 4H), 6.98-6.94 (comp, 2H), $4.20(\mathrm{~s}, 1 \mathrm{H}), 4.00$ (d, $J=14.1 \mathrm{~Hz}, 1 \mathrm{H}), 3.78-3.68$ (comp, 2H), 3.17 (ddd, $J=11.6,5.1,3.7 \mathrm{~Hz}, 1 \mathrm{H}$ ), 2.93 (ddd, $J=15.8,10.5,5.0 \mathrm{~Hz}, 1 \mathrm{H}$ ), 2.72 (app dt, $J$ $=16.2,3.6 \mathrm{~Hz}, 1 \mathrm{H}), 2.51(\mathrm{app} t \mathrm{td}, J=11.0,3.4 \mathrm{~Hz}, 1 \mathrm{H}), 1.98-1.91(\mathrm{~m}, 1 \mathrm{H}), 1.79-1.54(\mathrm{comp}$, $4 \mathrm{H}), 1.40-1.00$ (comp, 5H); ${ }^{13} \mathrm{C}$ NMR $\left(125 \mathrm{MHz}, \mathrm{CDCl}_{3}\right) \delta 171.8,142.1,134.0,132.5,128.7$, 127.6, 127.1, 126.9, 126.5, 126.4, 125.2, 68.2, 54.7, 48.2, 46.8, 33.2, 33.0, 29.0, 25.8, 25.1, 25.0; $\mathrm{m} / z$ (ESI-MS) $355.3[\mathrm{M}+\mathrm{H}]^{+}$.

$N$-cyclohexyl-2-(furan-2-ylmethyl)-1,2,3,4-tetrahydroisoquinoline-1-carboxamide $\quad 1 t:$

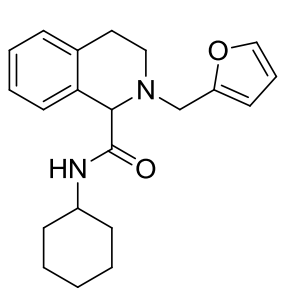

Following the general procedure B, cyclohexyl isocyanide, furan-2-carbaldehyde and 1,2,3,4-tetrahydroisoquinoline were heated at reflux for $3 \mathrm{~h}$. Product $\mathbf{1 t}$ was obtained as a yellow oil in $68 \%$ yield (115 $\mathrm{mg}),\left(\mathrm{R}_{\mathrm{f}}=0.19\right.$ in hexane/EtOAc 90:10 v/v); IR (KBr) 3370, 2930, 2853, $1671,1508,1452,1313,1149,1010,910,880,805,735 ;{ }^{1} \mathrm{H}$ NMR $\left(\mathrm{CDCl}_{3}\right.$, $500 \mathrm{MHz}) \delta$ 7.65-7.60 (m, 1H), 7.41-7.37 (m, 1H), 7. 31-7.27 (m, 1H), 7.18-7.12 (comp, 2H), 7.09-7.04 (m, 1H), 6.36-6.32 (m, 1H), 6.28-6.24 (m,1H), $4.18(\mathrm{~s}, 1 \mathrm{H})$, $3.82(\mathrm{~d}, J=14.4 \mathrm{~Hz}, 1 \mathrm{H}), 3.77-3.68(\mathrm{~m}, 1 \mathrm{H}), 3.56(\mathrm{~d}, J=14.5 \mathrm{~Hz}, 1 \mathrm{H}), 3.13$ (ddd, $J=11.2,5.0$, $3.1 \mathrm{~Hz}, 1 \mathrm{H}), 2.94$ (ddd, $J=16.1,11.2,5.1 \mathrm{~Hz}, 1 \mathrm{H}), 2.70$ (app dt, $J=16.1,3.3 \mathrm{~Hz}, 1 \mathrm{H}), 2.54$ (app td, $J=11.3,3.3 \mathrm{~Hz}, 1 \mathrm{H}), 1.99-1.91(\mathrm{~m}, 1 \mathrm{H}), 1.76-1.67$ (comp, 2H), 1.65-1.54 (comp, 2H), 1.42-1.11 (comp, 4H), 1.08-0.97(m, 1H); ${ }^{13} \mathrm{C}$ NMR (125 MHz, $\left.\mathrm{CDCl}_{3}\right) \delta 171.9,151.6,142.6$, 134.0, 132.7, 128.6, 127.5, 126.9, 126.4, 110.4, 109.4, 67.6, 52.5, 48.0, 47.2, 33.0, 29.4, 25.8, 25.0, 24.9; $\mathrm{m} / z$ (ESI-MS) $339.1[\mathrm{M}+\mathrm{H}]^{+}$.

2-benzyl- $N$-cyclohexyl-6,7-dimethoxy-1,2,3,4-tetrahydroisoquinoline-1-carboxamide 1u:

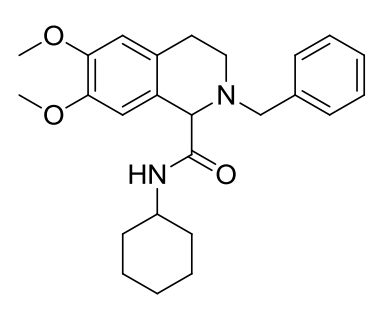

Following the general procedure B, cyclohexyl isocyanide, benzaldehyde and 6,7-dimethoxy-1,2,3,4-tetrahydroisoquinoline were heated at reflux for $3 \mathrm{~h}$. Product $1 \mathrm{u}$ was obtained as a white solid in 94\% yield (192 mg), $\left(\mathrm{R}_{\mathrm{f}}=0.21\right.$ in hexane/EtOAc 80:20 v/v); $\mathrm{mp}=135-137{ }^{\circ} \mathrm{C} ; \quad$ IR $(\mathrm{KBr})$ 3380, 3322, 2928, 2849, 1648, 1606, 1518, 1453, 1254, 1229, 1131, 1020, 755, 730, 697; ${ }^{1} \mathrm{H}$ NMR $\left(\mathrm{CDCl}_{3}, 500 \mathrm{MHz}\right) \delta$ 7.38-7.26 (comp, 5H), 
7.12-7.07 (comp, 2H), $6.54(\mathrm{~s}, 1 \mathrm{H}), 4.07(\mathrm{~s}, 1 \mathrm{H}), 3.92(\mathrm{~d}, J=13.5 \mathrm{~Hz}, 1 \mathrm{H}), 3.84(\mathrm{~s}, 3 \mathrm{H}), 3.83(\mathrm{~s}$, $3 \mathrm{H}), 3.76-3.67(\mathrm{~m}, 1 \mathrm{H}), 3.43(\mathrm{~d}, J=13.5 \mathrm{~Hz}, 1 \mathrm{H}), 3.03(\mathrm{ddd}, J=11.4,5.0,3.7 \mathrm{~Hz}, 1 \mathrm{H}), 2.82$ (ddd, $J=15.7,10.3,4.8 \mathrm{~Hz}, 1 \mathrm{H}$ ), 2.60 (app dt, $J=15.9,3.6 \mathrm{~Hz}, 1 \mathrm{H}$ ), 2.43 (app td, $J=11.0,3.5$ $\mathrm{Hz}, 1 \mathrm{H}), 1.93-1.85(\mathrm{~m}, 1 \mathrm{H}), 1.81-2.73(\mathrm{~m}, 1 \mathrm{H}), 1.70-1.52(\mathrm{comp}, 3 \mathrm{H}), 1.38-1.23$ (comp, $2 \mathrm{H}$ ), 1.39-1.24 (comp, 2H), 1.19-0.96 (comp, 3H); ${ }^{13} \mathrm{C} \mathrm{NMR}\left(125 \mathrm{MHz}, \mathrm{CDCl}_{3}\right) \delta$ 172.3, 148.1, 147.6, 138.1, 128.9, 128.7, 127.6, 126.1, 124.6, 111.1, 110.1, 68.2, 60.2, 56.1, 56.0, 48.0, 46.9, $33.2,33.1,28.4,25.8,25.0,24.9 ; \quad \mathrm{m} / \mathrm{z}$ (ESI-MS) $409.0[\mathrm{M}+\mathrm{H}]^{+}$.

N,2-dibenzyl-1,2,3,4-tetrahydroisoquinoline-1-carboxamide 1v: Following the general

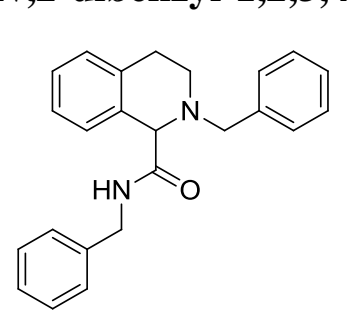

procedure B, benzyl isocyanide, benzaldehyde and 1,2,3,4-tetrahydroisoquinoline were heated at reflux for $3 \mathrm{~h}$. Product $\mathbf{1 v}$ was obtained as a yellow solid in $85 \%$ yield $(151 \mathrm{mg}),\left(\mathrm{R}_{\mathrm{f}}=0.22 \mathrm{in}\right.$ hexane/EtOAc 90:10 v/v); $\mathrm{mp}=130-131^{\circ} \mathrm{C} ; \quad \mathrm{IR}(\mathrm{KBr}) 3338,3029$, 2921, 2807, 1655, 1523, 1492, 1453, 1426, 1273, 1030, 755, 734, 696, 430; ${ }^{1} \mathrm{H}$ NMR $\left(\mathrm{CDCl}_{3}, 500 \mathrm{MHz}\right) \delta 7.71-7.67(\mathrm{~m}, 1 \mathrm{H}), 7.62-7.57(\mathrm{~m}, 1 \mathrm{H})$, 7.35-7.17 (comp, 12H), 7.16-7.11 (m, 1H), 4.63 (dd, $J=14.8,6.8 \mathrm{~Hz}, 1 \mathrm{H}), 4.36-4.28$ (comp, $2 \mathrm{H}), 3.95(\mathrm{~d}, J=13.4 \mathrm{~Hz}, 1 \mathrm{H}), 3.51(\mathrm{~d}, J=13.5 \mathrm{~Hz}, 1 \mathrm{H}), 3.11(\mathrm{ddd}, J=11.5,4.9,3.6 \mathrm{~Hz}, 1 \mathrm{H})$, 2.93 (ddd, $J=15.8,10.6,4.9 \mathrm{~Hz}, 1 \mathrm{H}$ ), 2.73 (app dt, $J=16.2,3.6 \mathrm{~Hz}, 1 \mathrm{H}), 2.50$ (app td, $J=11.1$, $3.4 \mathrm{~Hz}, 1 \mathrm{H}) ;{ }^{13} \mathrm{C} \mathrm{NMR}\left(125 \mathrm{MHz}, \mathrm{CDCl}_{3}\right) \delta 173.2,138.7,137.7,134.3,132.6,129.2,128.94$, 128.87, 128.7, 128.0, 127.8, 127.70, 127.68, 127.2, 126.5, 68.6, 60.4, 46.7, 43.5, 29.0; $\mathrm{m} / \mathrm{z}$ (ESI-MS) $357.2[\mathrm{M}+\mathrm{H}]^{+}$.

2-benzyl- $N$-phenyl-1,2,3,4-tetrahydroisoquinoline-1-carboxamide 1w: Following the

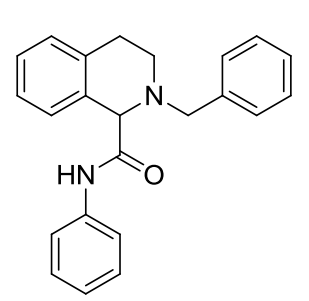

general procedure B, isocyanobenzene, benzaldehyde and 1,2,3,4-tetrahydroisoquinoline were heated at reflux for $3 \mathrm{~h}$. Product $\mathbf{1 w}$ was obtained as a white solid in $53 \%$ yield $(90 \mathrm{mg}),\left(\mathrm{R}_{\mathrm{f}}=0.45\right.$ in hexane/EtOAc 90:10 v/v); $\mathrm{mp}=117-119^{\circ} \mathrm{C} ; \quad \mathrm{IR}(\mathrm{KBr}) 3328,2813,1676$, $1604,1523,1493,1439,1313,1153,1128,755,747,692,535 ; \quad{ }^{1} \mathrm{H}$ NMR $\left(\mathrm{CDCl}_{3}, 500 \mathrm{MHz}\right) \delta 9.28(\mathrm{~s}, 1 \mathrm{H}), 7.69-7.64(\mathrm{~m}, 1 \mathrm{H}), 7.58-7.53$ (comp, 2H), 7.43-7.28 (comp, 7H), 7.24-7.18 (comp, 2H), 7.15-7.05 (comp, 2H), 4.35 (s, 1H), 4.01 (d, $J=$ $13.6 \mathrm{~Hz}, 1 \mathrm{H}), 3.61(\mathrm{~d}, J=13.5 \mathrm{~Hz}, 1 \mathrm{H}), 3.20(\mathrm{ddd}, J=11.6,5.0,3.9 \mathrm{~Hz}, 1 \mathrm{H}), 2.99$ (ddd, $J=15.8$, $10.5,5.0 \mathrm{~Hz}, 1 \mathrm{H}), 2.79$ (app dt, $J=16.2,3.6 \mathrm{~Hz}, 1 \mathrm{H}), 2.59(\operatorname{app~td}, J=11.1,3.4 \mathrm{~Hz}, 1 \mathrm{H}) ;{ }^{13} \mathrm{C}$ NMR $\left(125 \mathrm{MHz}, \mathrm{CDCl}_{3}\right) \delta 171.1,138.1,137.5,134.3,131.8,129.23,129.15,128.92,128.88$, $127.90,127.85,127.4,126.6,124.3,119.6,68.9,60.6,46.7,28.9 ; \quad \mathrm{m} / z(\mathrm{ESI}-\mathrm{MS}) 343.1[\mathrm{M}+$ $\mathrm{H}]^{+}$. 
$N$-cyclohexyl-1,2,3,4-tetrahydroisoquinoline-1-carboxamide (2) and $N$-cyclohexyl-2-ethyl-1,2,3,4-tetrahydroisoquinoline-1-carboxamide (3):

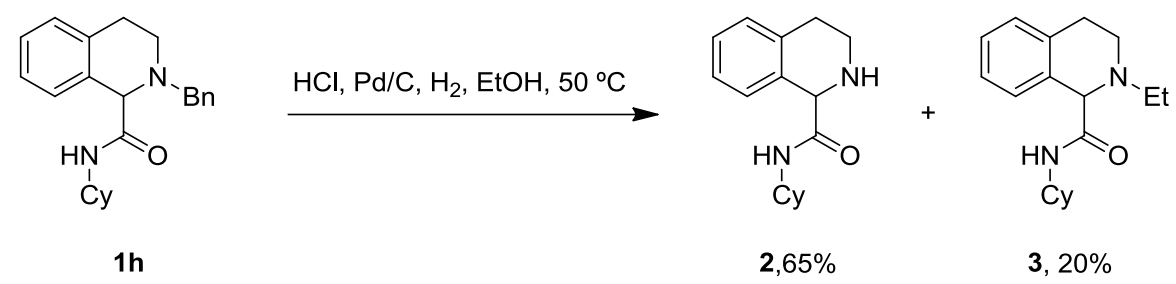

Following a modified literature procedure, ${ }^{3}$ a solution of $\mathbf{1 h}(62 \mathrm{mg}, 0.18 \mathrm{mmol})$ in $\mathrm{MeOH}(5 \mathrm{~mL})$ was treated with methanolic $\mathrm{HCl}$ (1.2 equiv). The solvent was subsequently removed under reduced pressure to obtain the hydrochloride salt, which was dissolved in $\mathrm{EtOH}(5 \mathrm{~mL})$. To this solution was added $10 \% \mathrm{Pd} / \mathrm{C}(10 \mathrm{mg})$ and it was stirred for $20 \mathrm{~h}$ at $50{ }^{\circ} \mathrm{C}$ under an atmosphere of hydrogen. The reaction mixture was allowed to cool to room temperature and filtered through Celite. The solvent was removed under reduced pressure and the residue purified by flash chromatography on silica gel. Product 2 was obtained as a white solid in $65 \%$ yield (30 $\mathrm{mg}),\left(\mathrm{R}_{\mathrm{f}}=0.52\right.$ in EtOAc/MeOH 90:10 v/v); $\mathrm{mp}=130-132{ }^{\circ} \mathrm{C} ; \quad \mathrm{IR}(\mathrm{KBr}) 3283,3057,3017$,

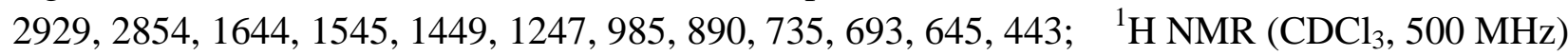
א 7.54-7.49 (m, 1H), 7.19-7.14 (comp, 2H), 7.10-7.06 (m, 1H), 7.04-6.96 (m, 1H), 4.50 (s, 1H), 3.78-3.68 (m, 1H), 3.12-3.03 (comp, 2H), 2.89-2.81 (m, 1H), 2.79-2.70 (m, 1H), 2.16 (br s, 1H) 1.93-1.86 (m, 1H), 1.83-1.75 (m, 1H), 1.71-1.53 (comp, 3H), 1.41-1.25 (comp, 2H), 1.20-1.02 (comp, 3H); ${ }^{13} \mathrm{C}$ NMR $\left(125 \mathrm{MHz}, \mathrm{CDCl}_{3}\right) \delta 171.9,134.8,133.1,129.2,128.1,127.1,126.1$, 60.4, 48.1, 41.5, 33.2, 33.1, 29.6, 25.8, 25.0, 24.9.; $\mathrm{m} / z$ (ESI-MS) $259.1[\mathrm{M}+\mathrm{H}]^{+}$.

In addition, product 3 was obtained as a white solid in $20 \%$ yield $(10 \mathrm{mg}),\left(\mathrm{R}_{\mathrm{f}}=0.12\right.$ in hexane/EtOAc 90:10 v/v); $\mathrm{mp}=88-89^{\circ} \mathrm{C} ; \quad \mathrm{IR}(\mathrm{KBr}) 3275,3067,2931,2852,1637,1536$, 1448, 1248, 1095, 930, 888, 735; ${ }^{1} \mathrm{H} \mathrm{NMR}\left(\mathrm{CDCl}_{3}, 500 \mathrm{MHz}\right) \delta 7.60-7.56(\mathrm{~m}, 1 \mathrm{H}), 7.17-7.05$ (comp, 4H), 4.06 (s, 1H), 3.75-3.65 (m, 1H), 3.17 (ddd, $J=11.3,4.9,3.2 \mathrm{~Hz}, 1 \mathrm{H}), 2.95$ (ddd, $J=$ 16.0, 11.2, 4.9 Hz, 1H), 2.77-2.65 (comp, 2H), 2.54-2.43 (comp, 2H), 1.94-1.89 (m, 1H), 1.721.53 (comp, 5H), 1.41-1.24 (comp, 2H), 1.20-1.09 (comp, 4H), 1.02-0.94 (m, 1H); ${ }^{13} \mathrm{C}$ NMR $\left(125 \mathrm{MHz}, \mathrm{CDCl}_{3}\right) \delta 172.6,134.3,133.1,128.5,127.5,126.8,126.3,68.5,50.3,47.8,46.5,33.2$, 33.0, 29.5, 25.8, 25.0, 24.9, 12.6.; $\mathrm{m} / z$ (ESI-MS) $287.1[\mathrm{M}+\mathrm{H}]^{+}$.

$\mathrm{N}$-cyclohexylisoquinoline-1-carboxamide 4: Following a modified literature procedure, ${ }^{4}$ to a solution of $1 \mathbf{k}(76 \mathrm{mg}, 0.2 \mathrm{mmol})$ in mesitylene $(2 \mathrm{~mL}, 0.1 \mathrm{M})$ was added $10 \% \mathrm{Pd} / \mathrm{C}$ $(43 \mathrm{mg}$ ) and the mixture was stirred under reflux for $4 \mathrm{~h}$. The reaction mixture was allowed to cool to room temperature, diluted with $5 \mathrm{~mL}$ of EtOAc and a few drops of triethylamine, and then filtered through Celite. The solvent was removed under reduced pressure and the residue purified by flash chromatography on silica gel. Product 4 was obtained as a white solid in $71 \%$ yield $(36 \mathrm{mg}),\left(R_{\mathrm{f}}=0.45\right.$ in 
hexane/EtOAc 90:10 v/v); $\quad \mathrm{mp}=118-120^{\circ} \mathrm{C} ; \quad \operatorname{IR}(\mathrm{KBr}) 3355,2923,2856,1654,1513,1451$, $1383,1341,1313,1251,1148,763,625,513 ;{ }^{1} \mathrm{H}$ NMR $\left(\mathrm{CDCl}_{3}, 500 \mathrm{MHz}\right) \delta 9.62-9.59(\mathrm{~m}$, $1 \mathrm{H}), 8.44(\mathrm{~d}, J=5.5 \mathrm{~Hz}, 1 \mathrm{H}), 8.14-8.06(\mathrm{~m}, 1 \mathrm{H}), 7.84-7.80(\mathrm{~m}, 1 \mathrm{H}), 7.78-7.74(\mathrm{~m}, 1 \mathrm{H}), 7.72-$ 7.63 (comp, 2H), 4.06-3.97 (m, 1H), 2.11-2.04 (comp, 2H), 1.83-1.75 (comp, 2H), 1.70-1.62 $(\mathrm{m}, 1 \mathrm{H}), 1.52-1.32(\mathrm{comp}, 4 \mathrm{H}), 1.30-1.20(\mathrm{~m}, 1 \mathrm{H}) ;{ }^{13} \mathrm{C} \mathrm{NMR}\left(125 \mathrm{MHz}, \mathrm{CDCl}_{3}\right) \delta 165.4$, $148.9,140.4,137.6,130.6,128.7,128.2,127.3,126.9,124.3,48.5,33.3,25.9,25.2 . ; \quad \mathrm{m} / z(\mathrm{ESI}-$ MS) $277.6[\mathrm{M}+\mathrm{Na}]^{+}$. 
2D-NMR Analysis for Compound 1u, Selected Interactions (in $\mathrm{CDCl}_{3}$ ):

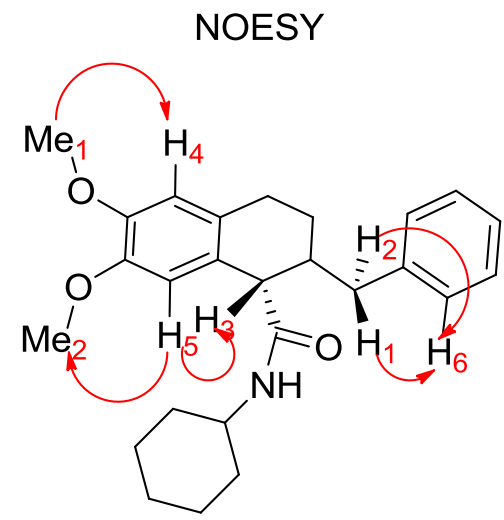

\begin{tabular}{|c|c|}
\hline Protons & Chemical Shifts (ppm) \\
\hline H1,H2 & $3.43,3.92$ \\
\hline Me1 & 3.83 \\
\hline Me2 & 3.84 \\
\hline H3 & 4.07 \\
\hline H4 & 6.54 \\
\hline H5 & $7.12-7.07$ \\
\hline H6 & $7.38-7.26$ \\
\hline
\end{tabular}




\section{References:}

1. Sehlinge, A.; Espinosa, L.; Meier, M. Macromolecules 2013, 46, 6031.

2. Janza, B.; Studer, A. Org. Lett. 2006, 8, 1875.

3. Nussbaumer, P.; Dechat. T, Monatsh. Chem. 2001, 132, 1047.

4. Teske, J.; Deiters, A. J. Org. Chem. 2008, 73, 342.

5. Popovici-Muller, J.; Saunders, J.; Salituro, F.; Travins, J.; Yan, S.; Zhao, F.; Gross, S.; Dang, L.; Yen, K.; Yang, H.; Straley, K.; Jin, S.; Kunii, K.; Fantin, V.; Zhang, S.; Pan, Q.; Shi, D.; Biller, S.; Su, S. ACS Med. Chem. Lett. 2012, 3, 850.

6. Fañanás-Mastral, M.; Teichert, J.; Fernández-Salas, J.; Heijnen, D.; Feringa, B. Org. Biomol. Chem. 2013, $11,4521$. 


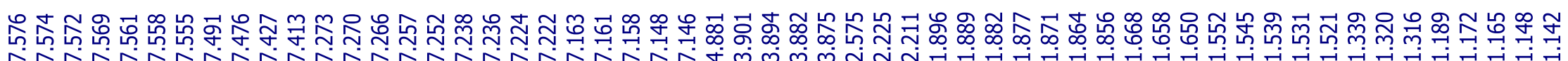

${ }^{1} \mathrm{H}$ NMR of $1 \mathrm{a}$ in $\mathrm{CDCl}_{3}$
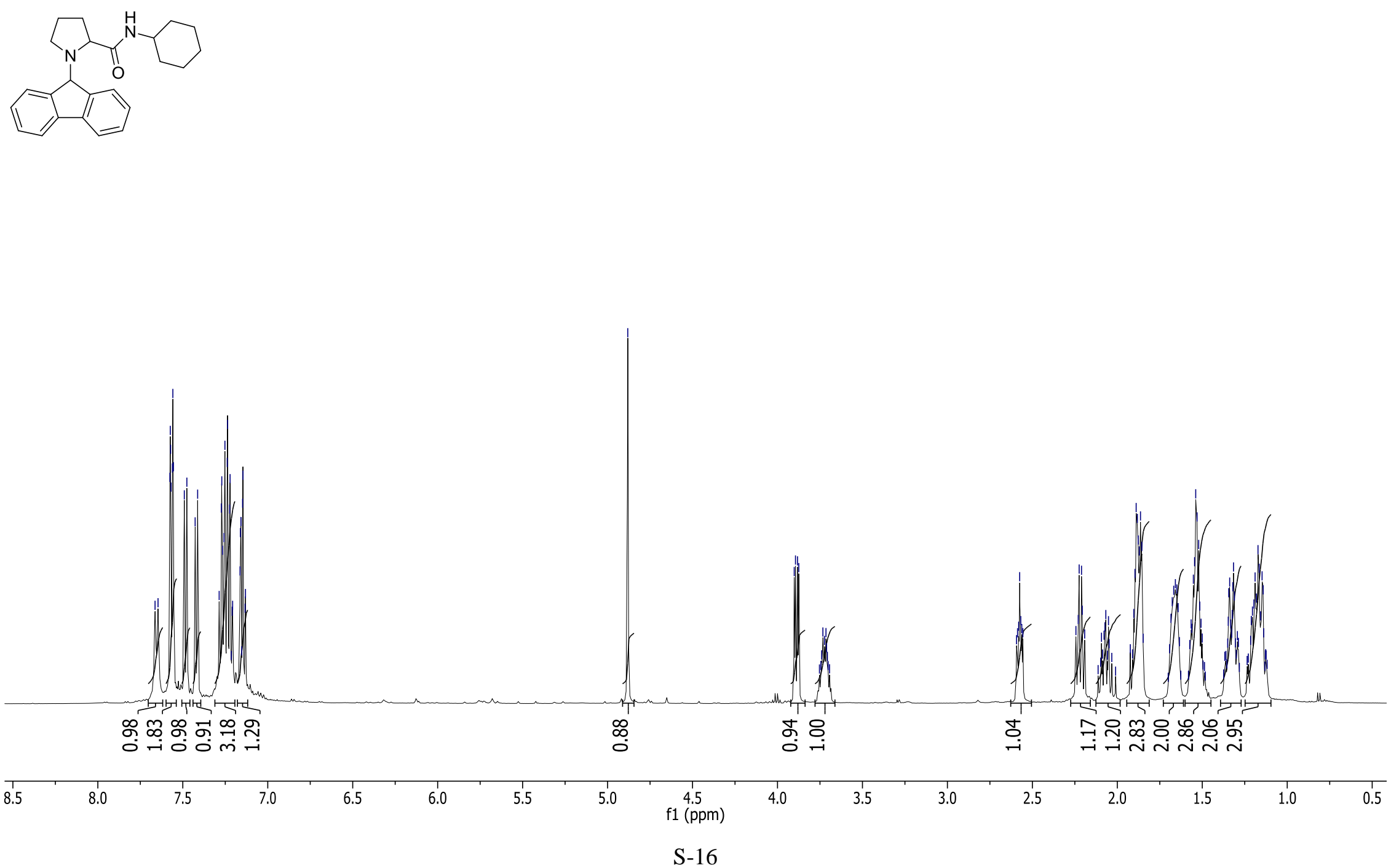


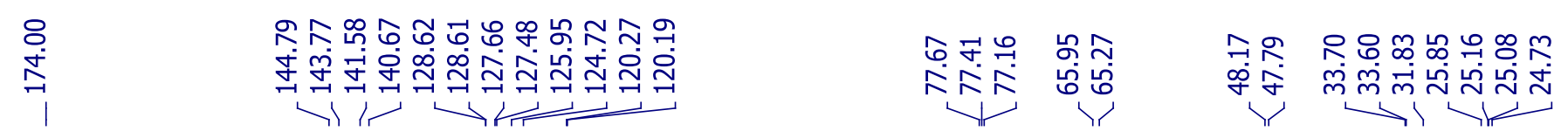

${ }^{13} \mathrm{C} \mathrm{NMR}$ of $1 \mathrm{a}$ in $\mathrm{CDCl}_{3}$
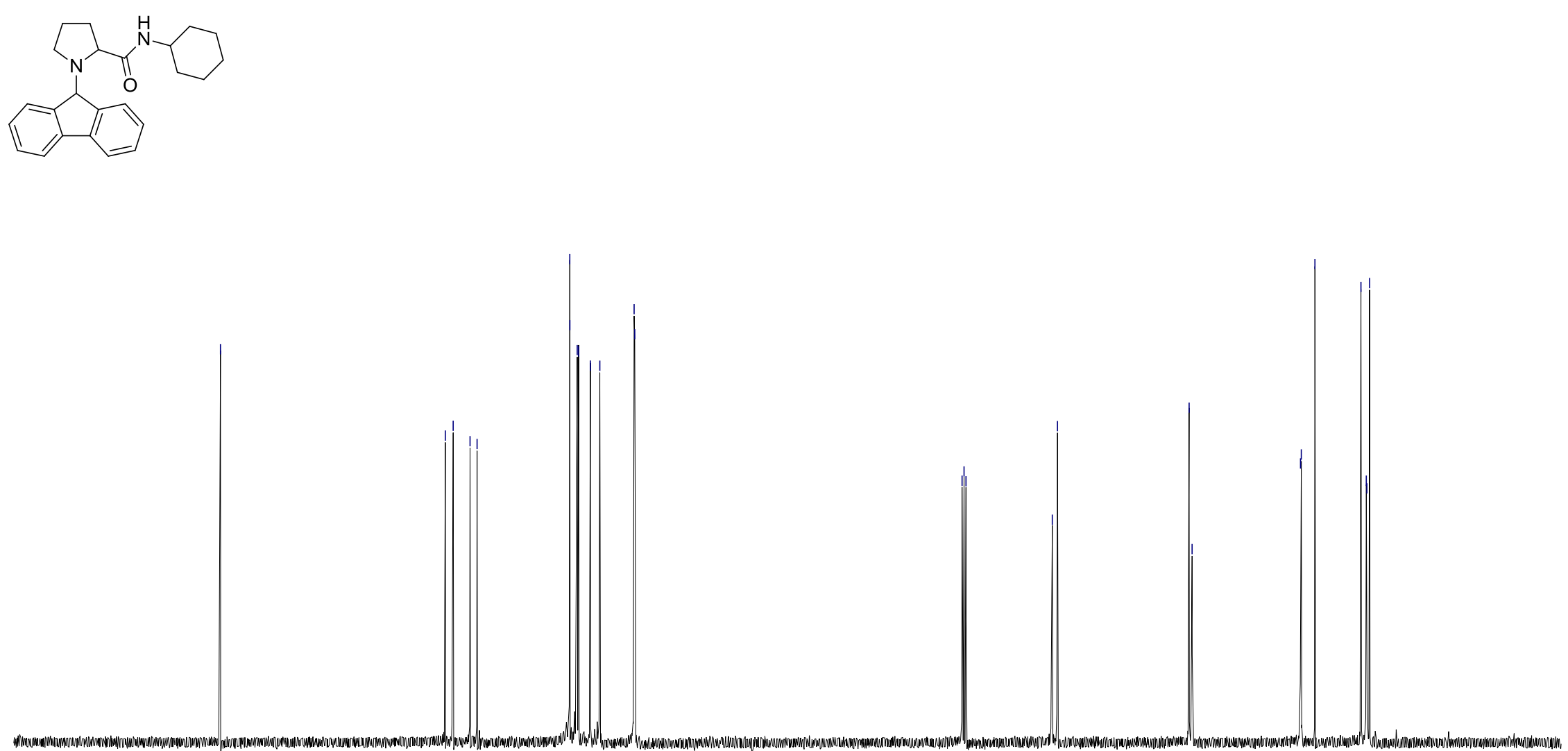

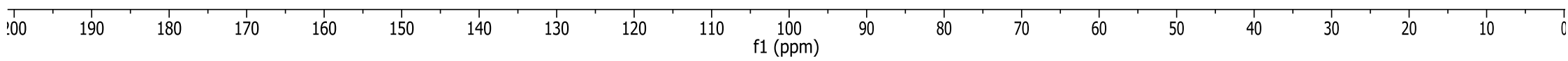




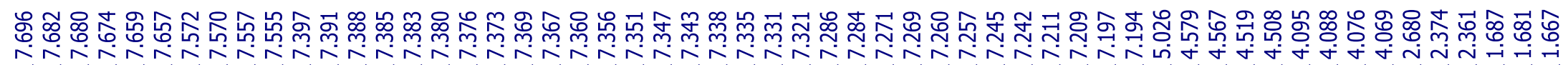

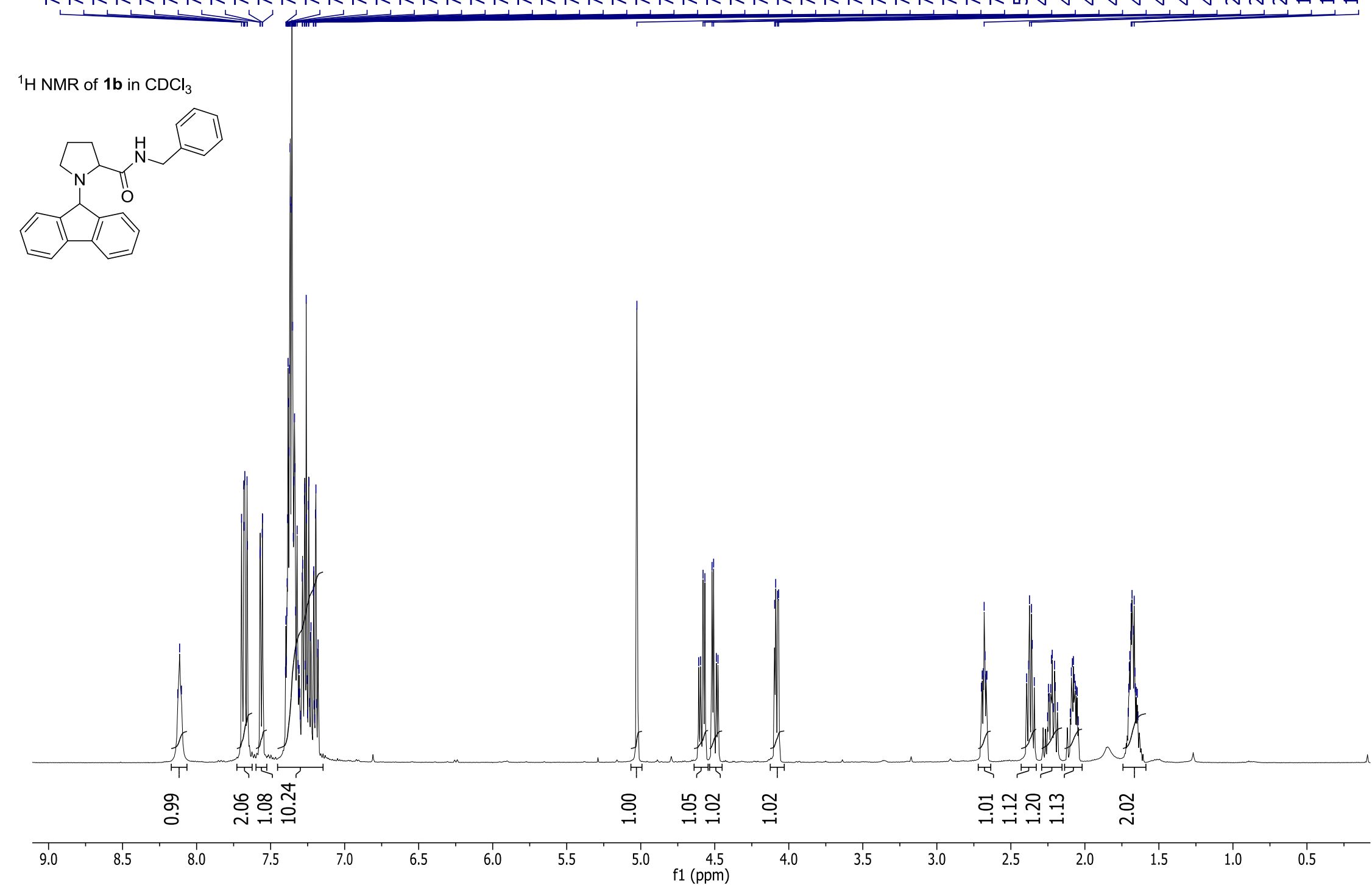

S-18 

命

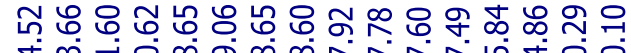

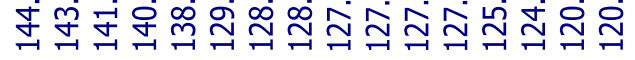

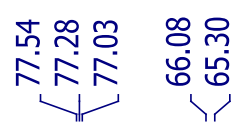
लू भ̊n

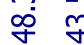

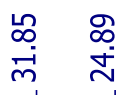

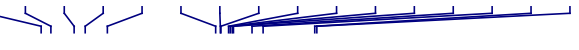
|
I 1

${ }^{13} \mathrm{C} \mathrm{NMR}$ of $1 \mathrm{~b}$ in $\mathrm{CDCl}_{3}$

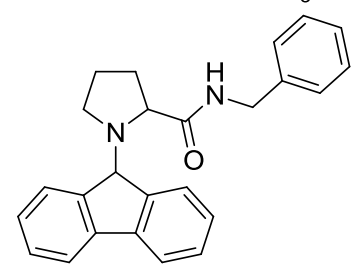

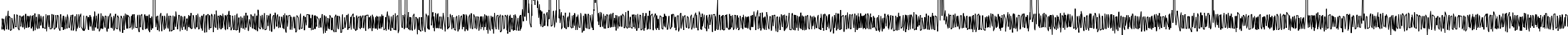

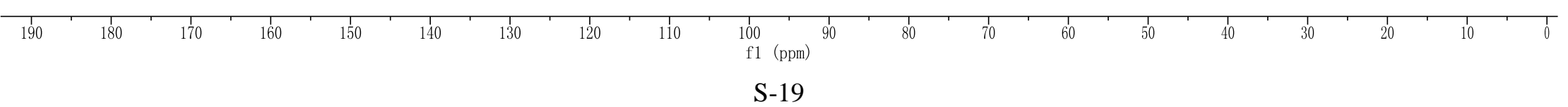




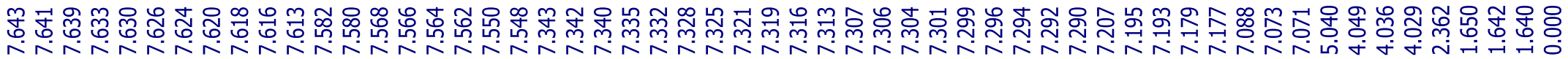

${ }^{1} \mathrm{H} \mathrm{NMR}$ of $1 \mathrm{c}$ in $\mathrm{CDCl}_{3}$
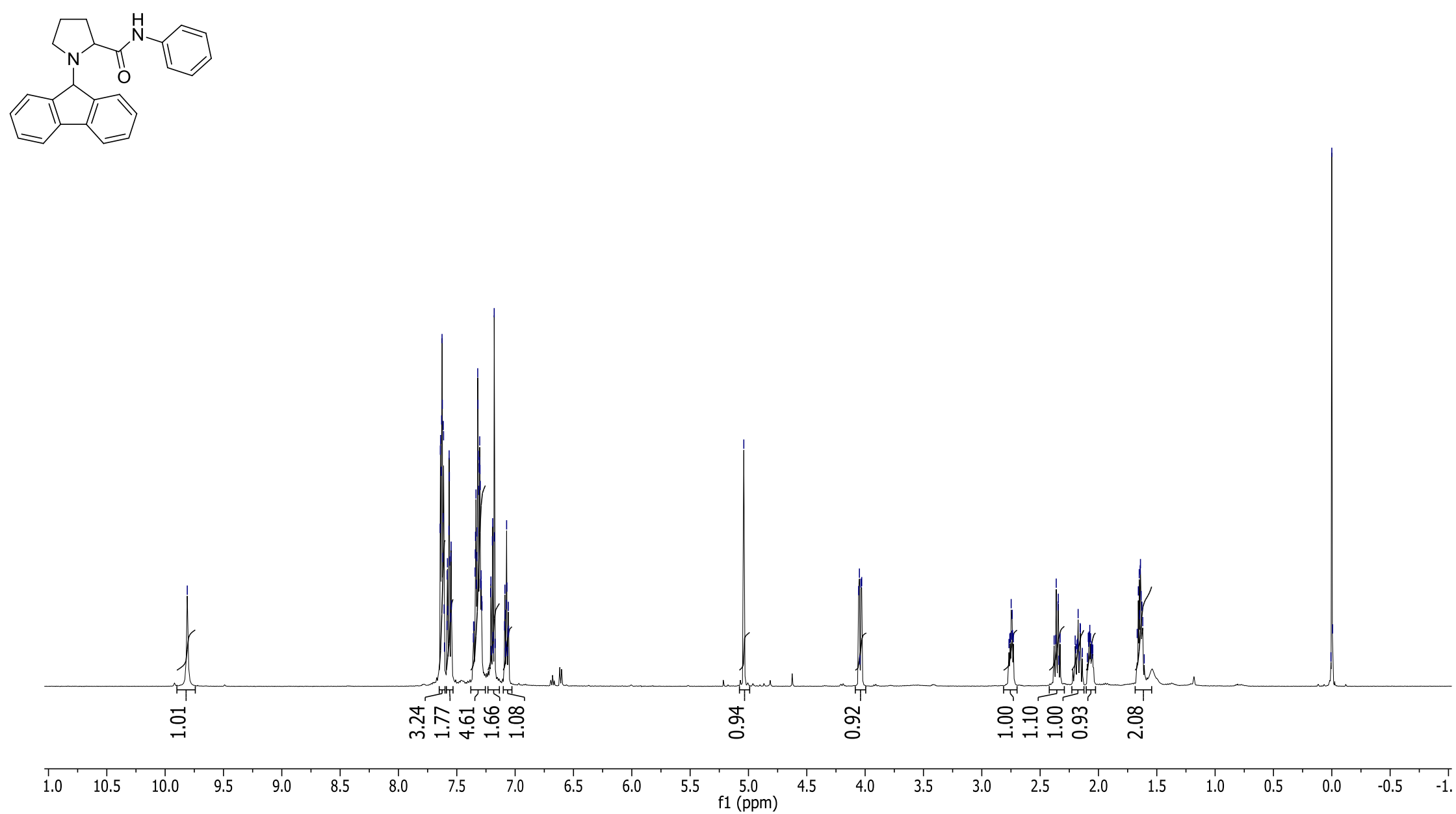
${ }^{13} \mathrm{C} \mathrm{NMR}$ of $1 \mathrm{c}$ in $\mathrm{CDCl}_{3}$
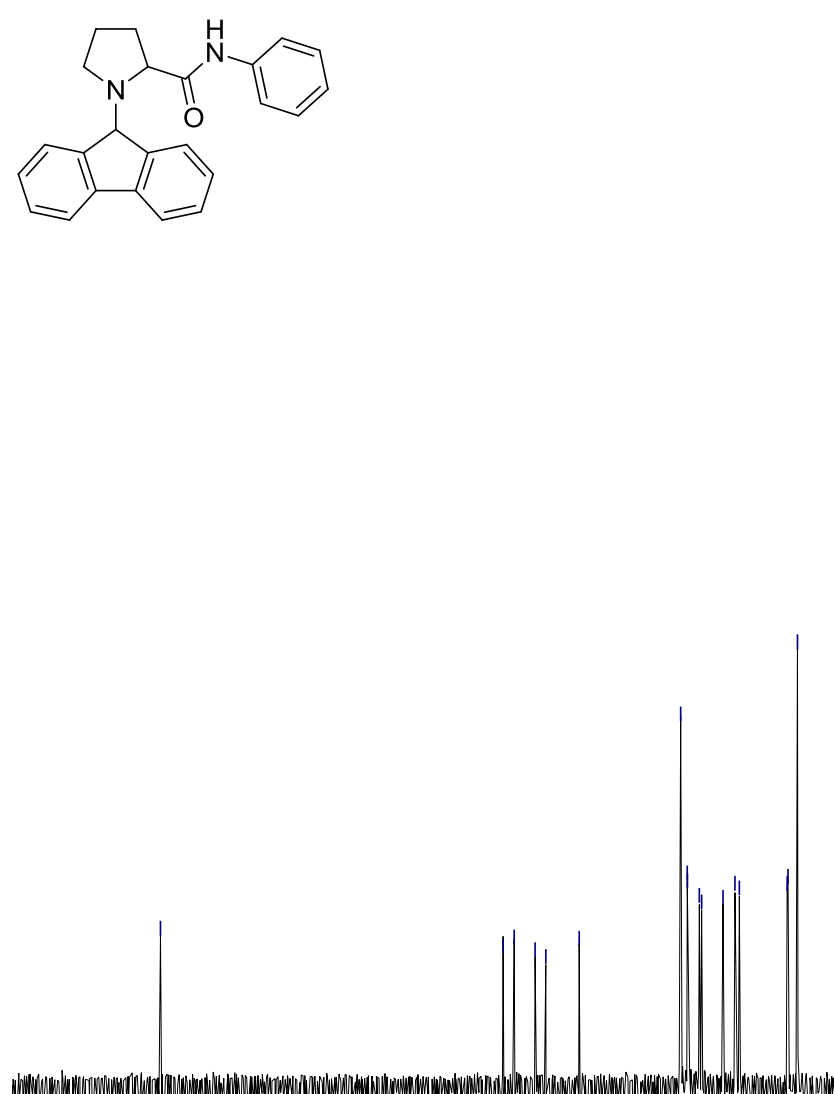

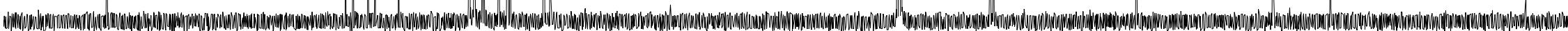
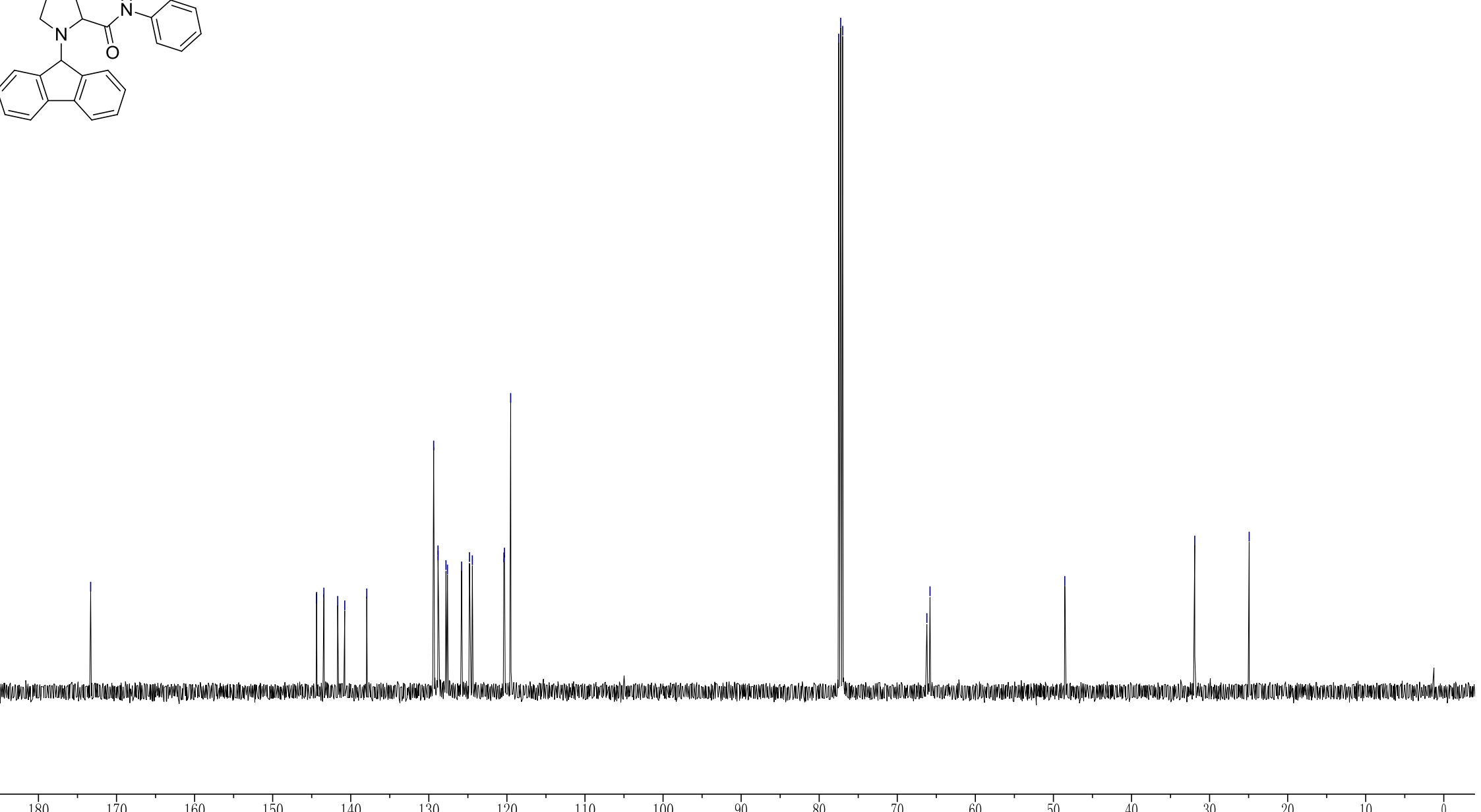
170 160 150 140 130 120 110 100 90 80 $70 \quad 60$ 40 30 10 


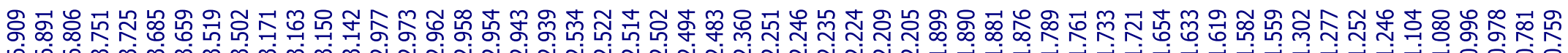
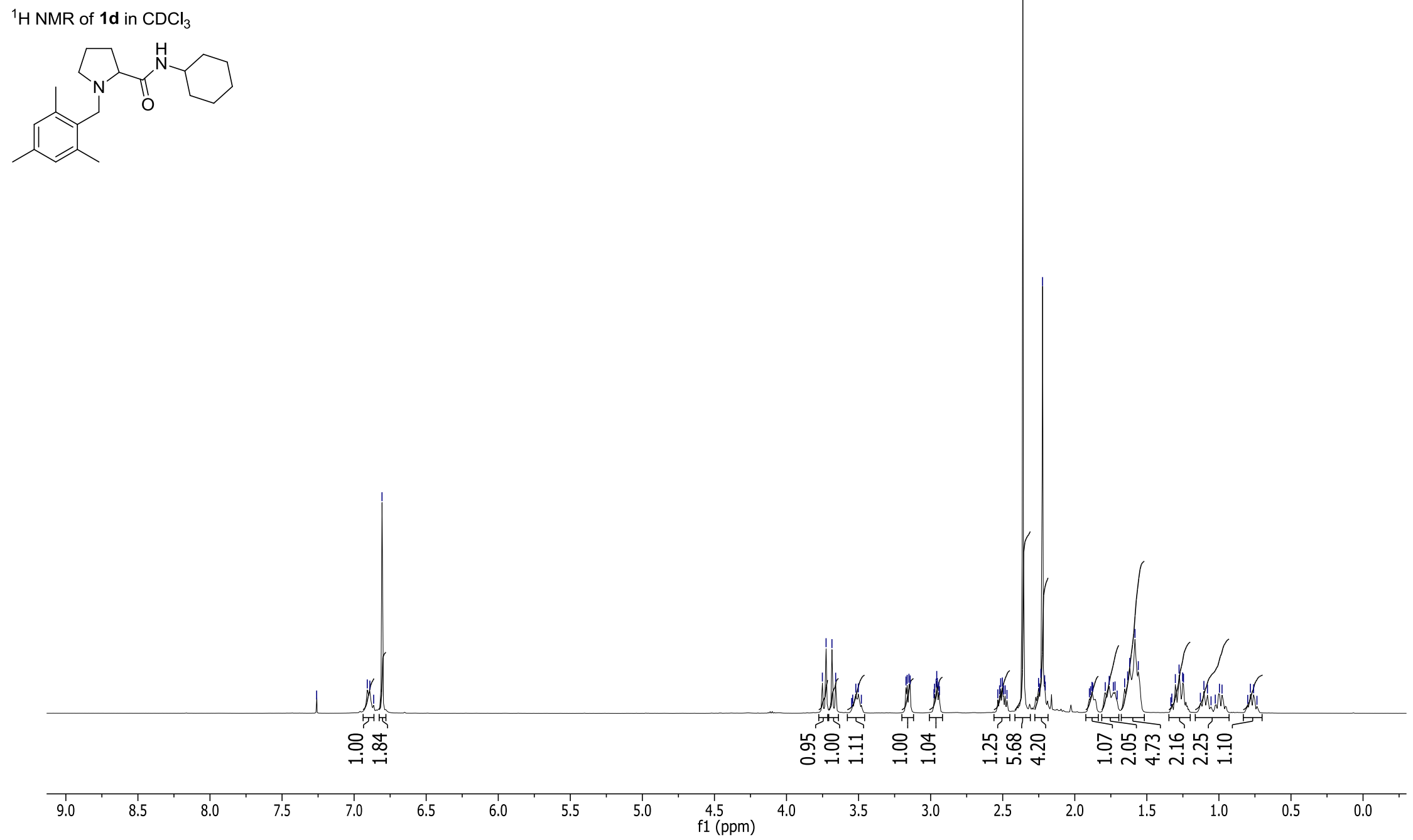


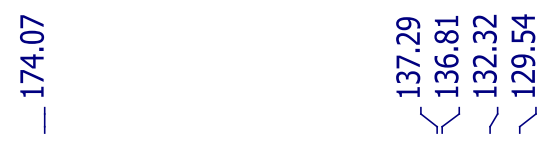

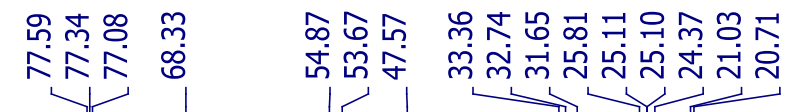

${ }^{13} \mathrm{C}$ NMR of $\mathbf{1 d}$ in $\mathrm{CDCl}_{3}$
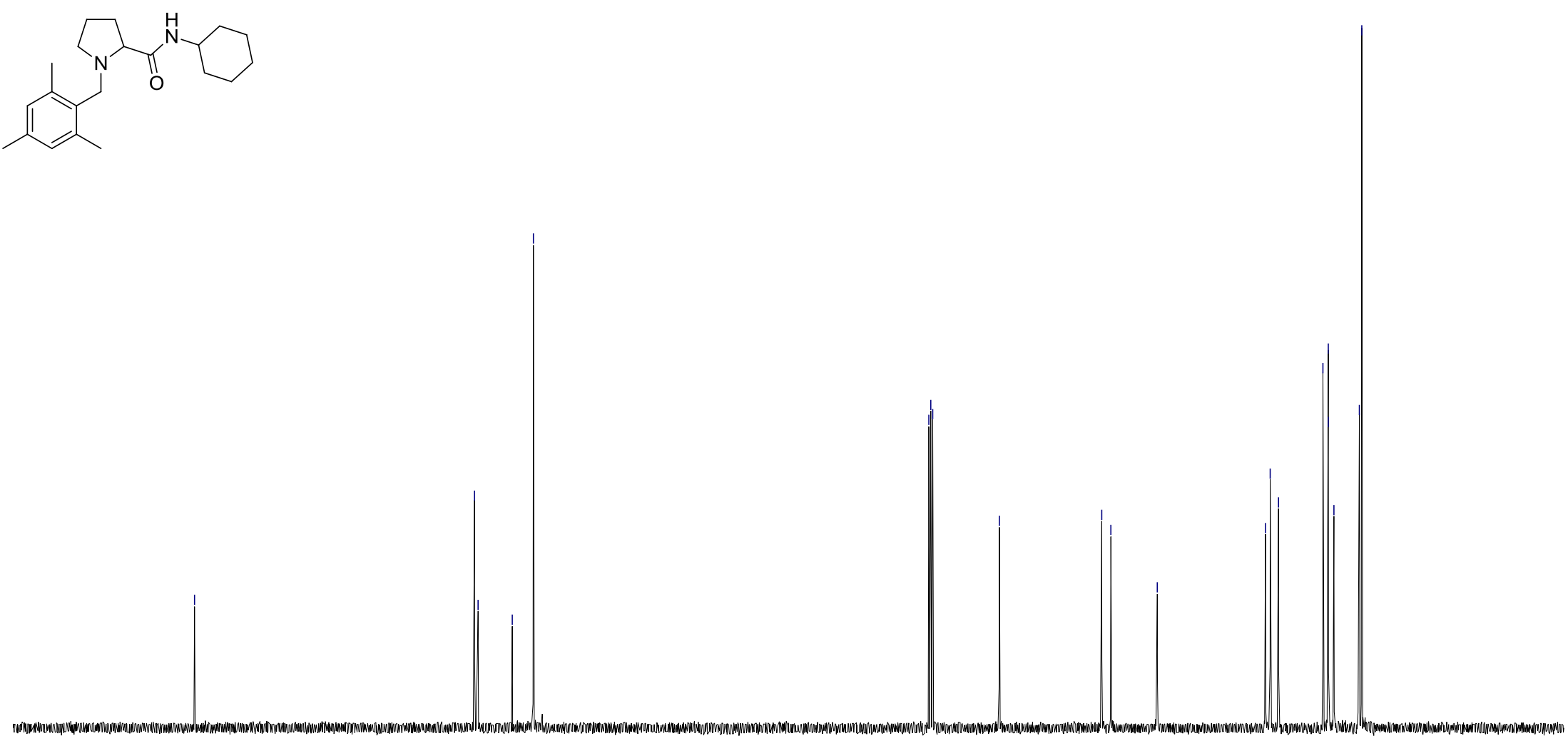


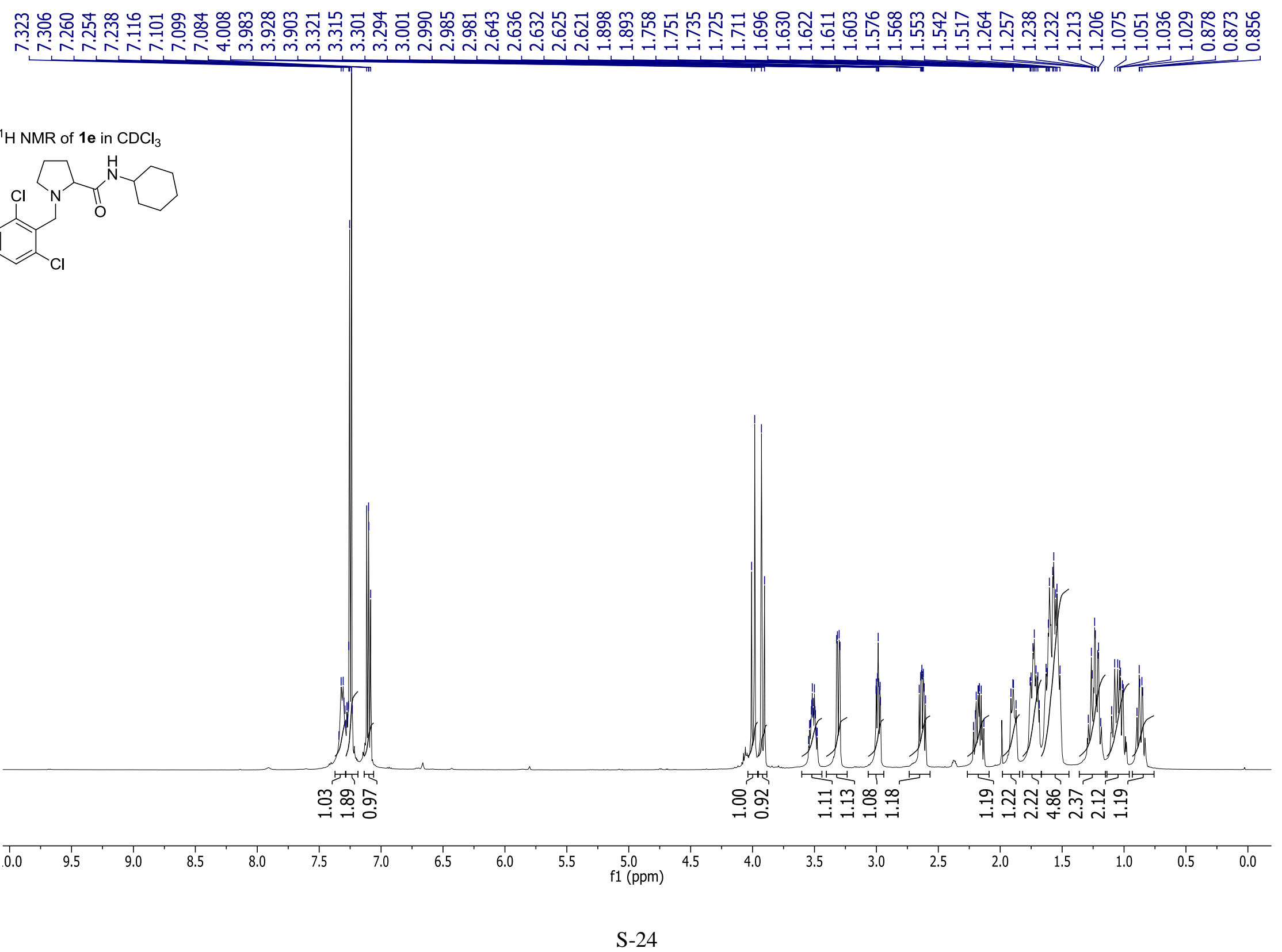




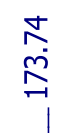
นำำ

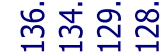

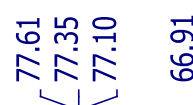

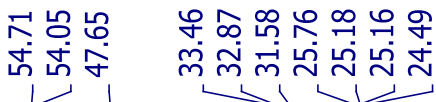

${ }^{13} \mathrm{C} \mathrm{NMR}$ of $1 \mathbf{e}$ in $\mathrm{CDCl}_{3}$

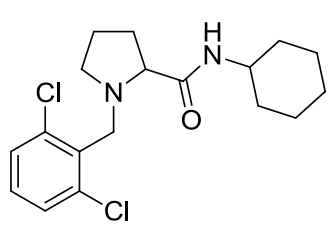

$\mathrm{C}$

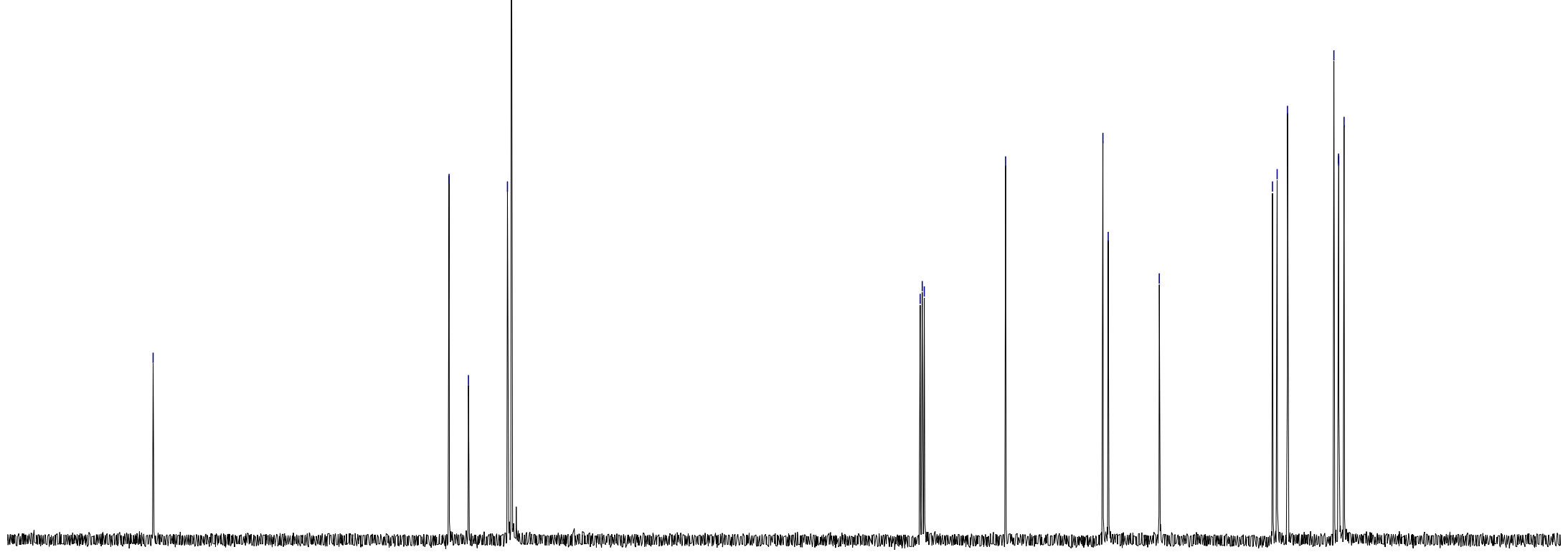

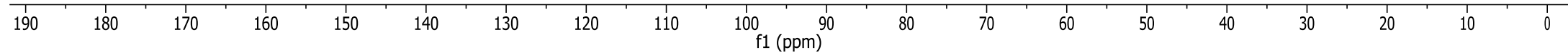


${ }^{1} \mathrm{H}$ NMR of $1 \mathrm{f}$ in $\mathrm{CDCl}_{3}$
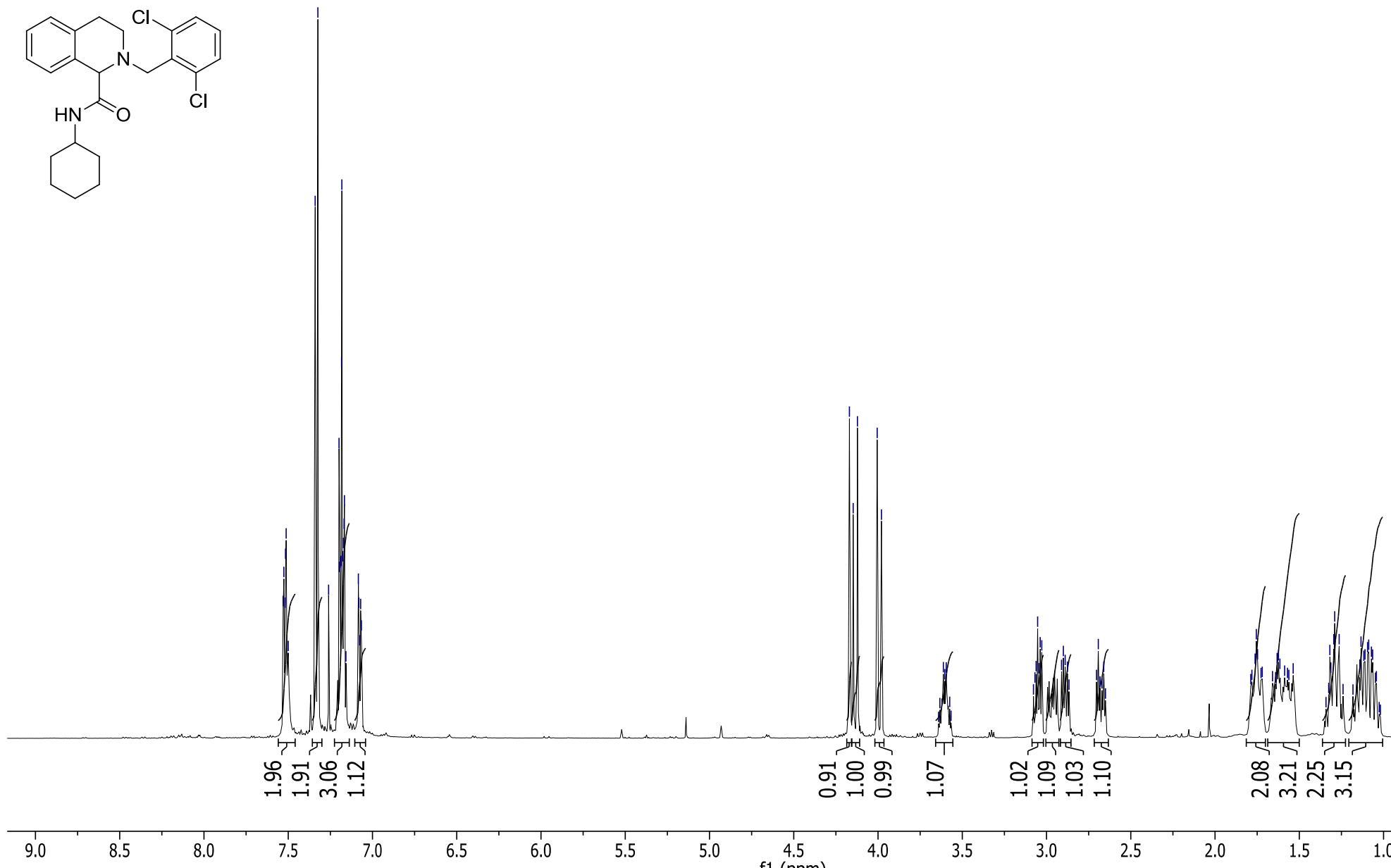

3.5

2.0

S-26 

옹

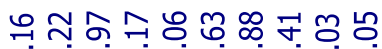

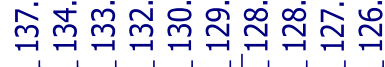

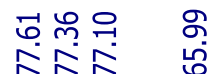
氛
국
ஸ่

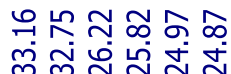
舟
$\longrightarrow$

${ }^{13} \mathrm{C} \mathrm{NMR}$ of $\mathbf{1 g}$ in $\mathrm{CDCl}_{3}$
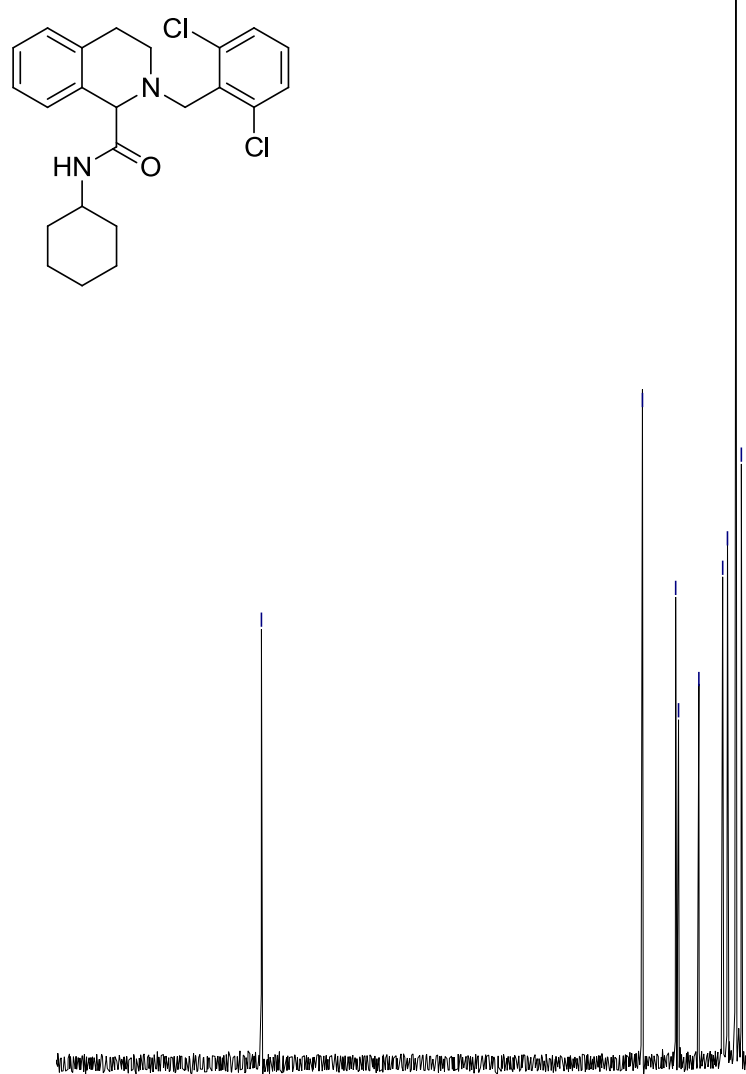

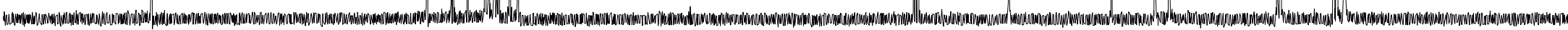




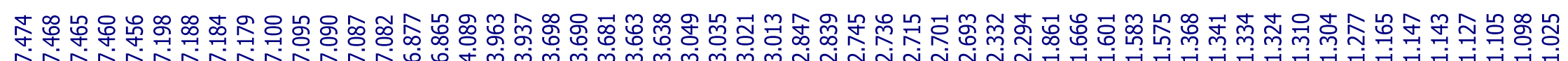

${ }^{1} \mathrm{H} \mathrm{NMR}$ of $1 \mathrm{~g}$ in $\mathrm{CDCl}_{3}$

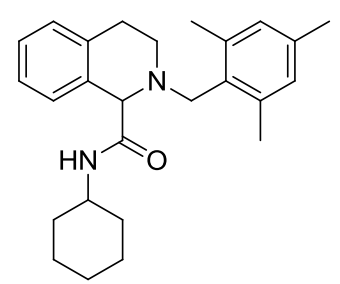

IIIIIIII) 


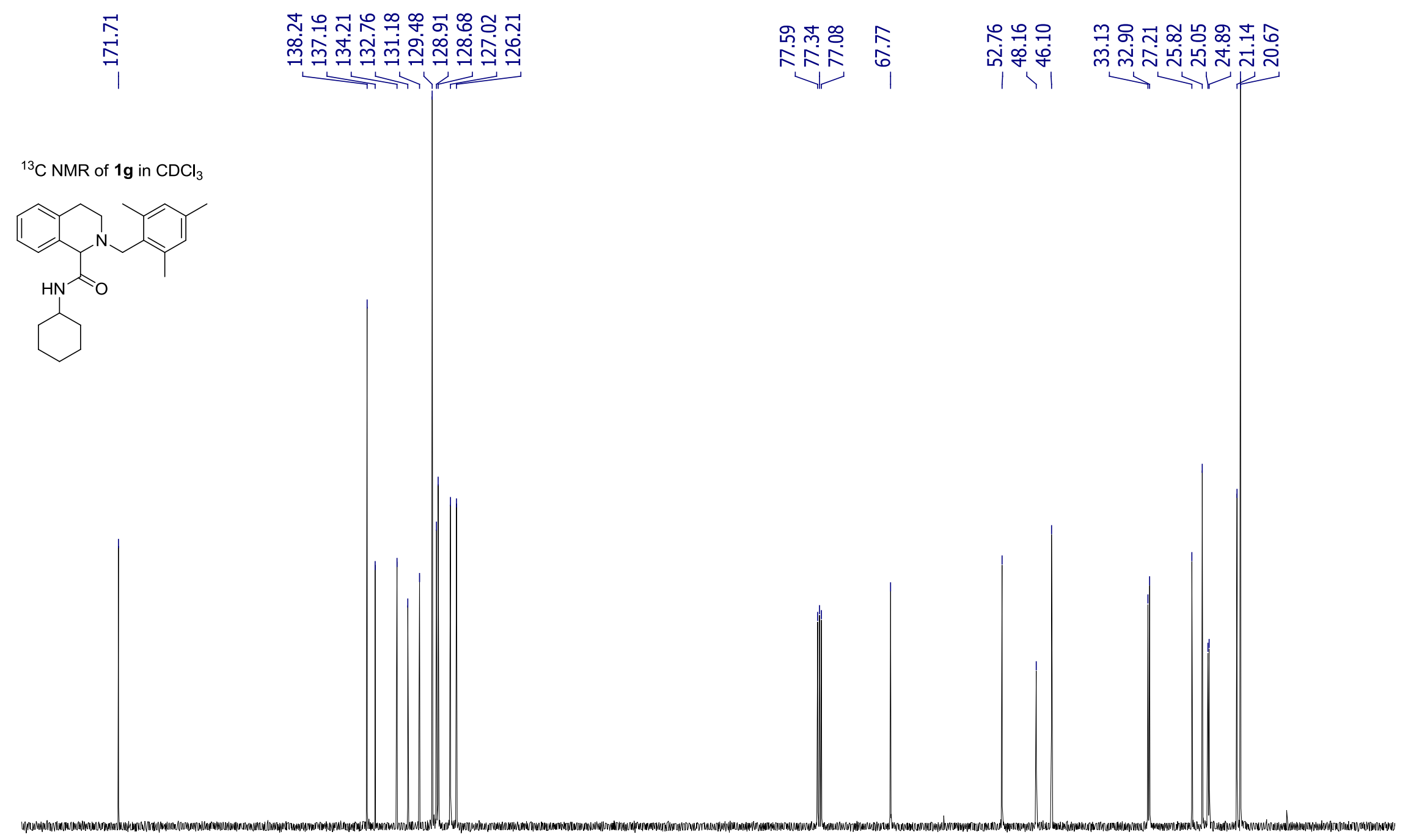

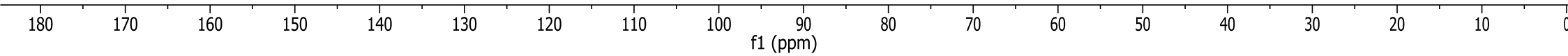




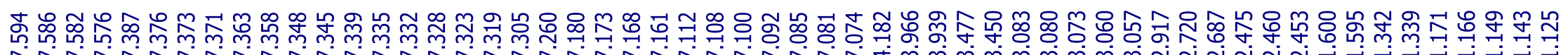

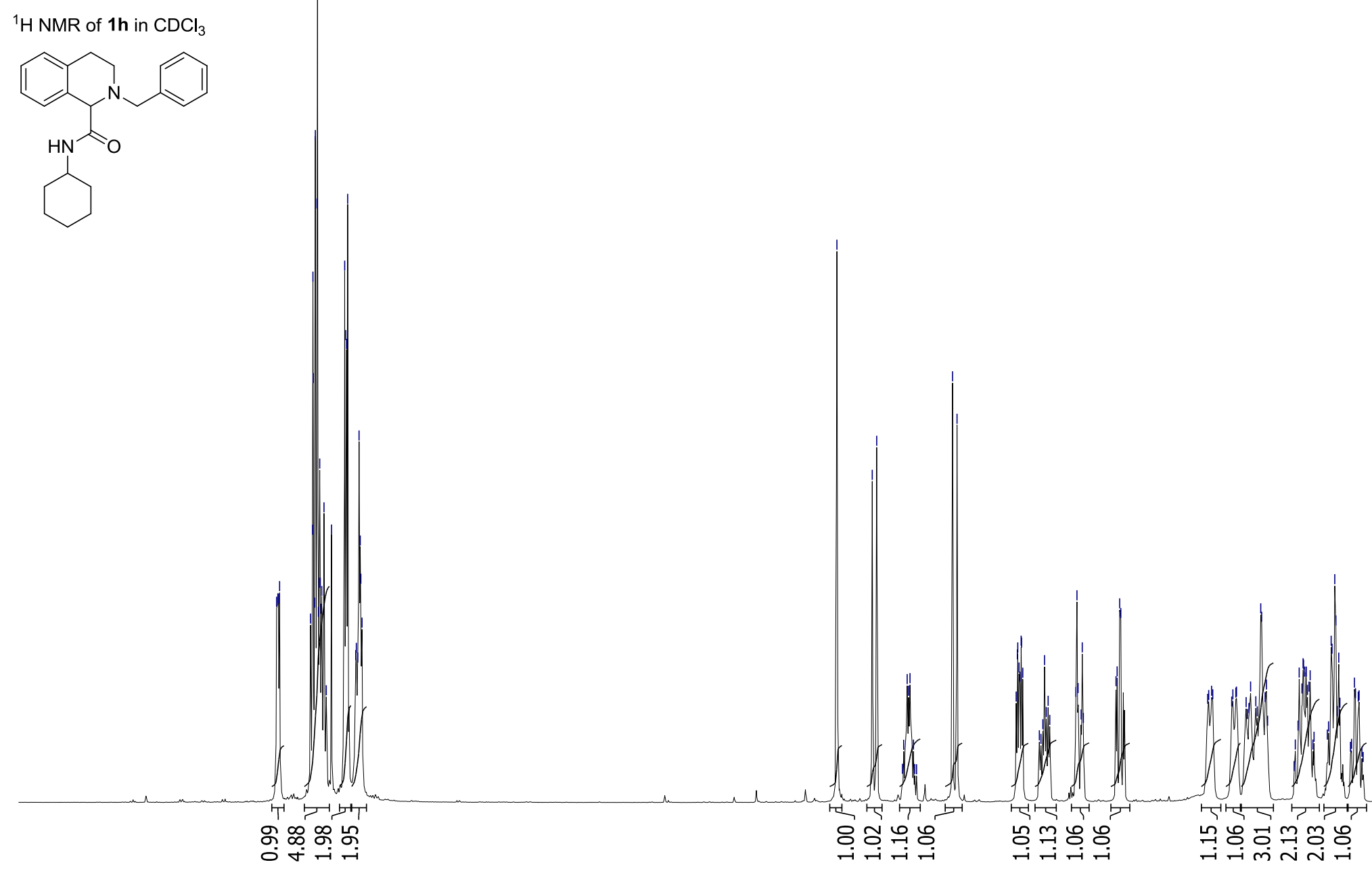


${ }^{13} \mathrm{C} \mathrm{NMR}$ of $1 \mathrm{~h}$ in $\mathrm{CDCl}_{3}$
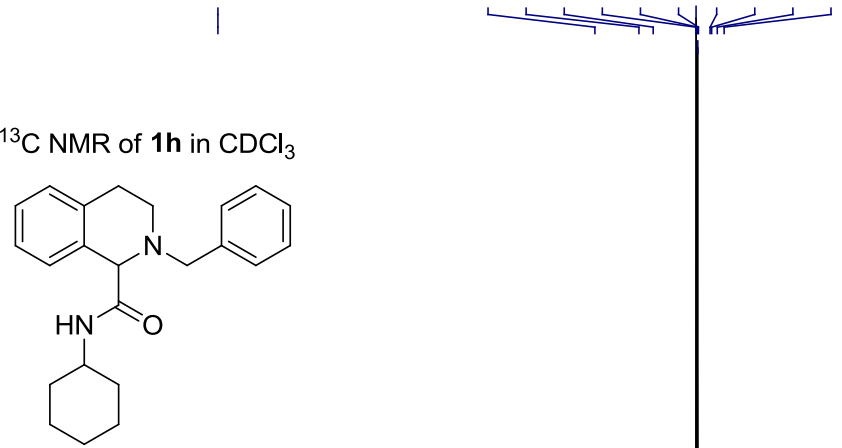

$\rightarrow<1<$

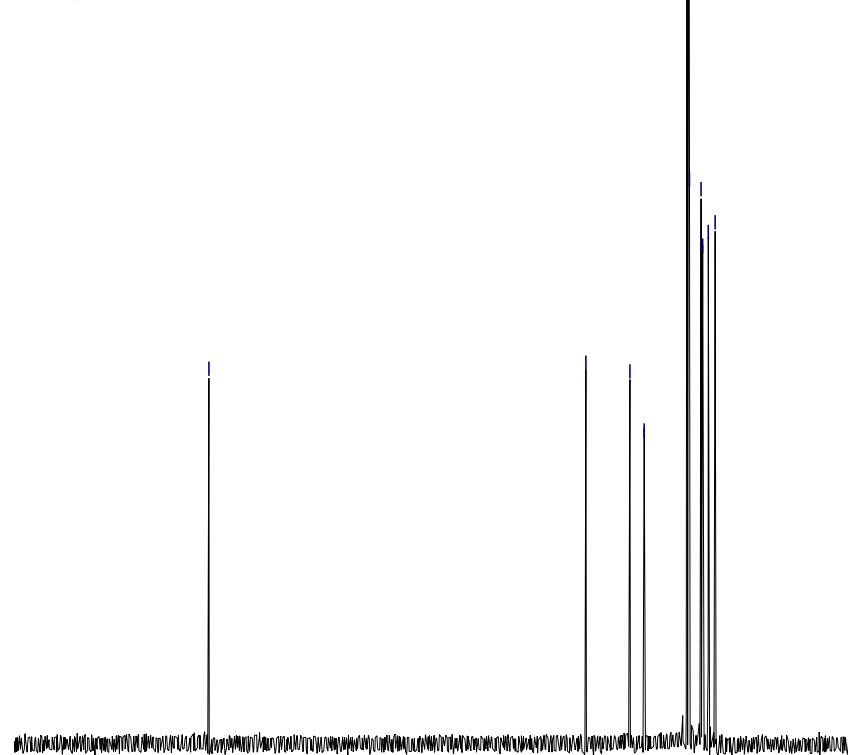

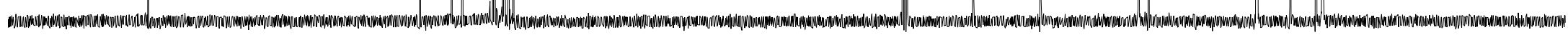




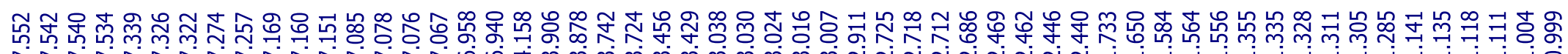

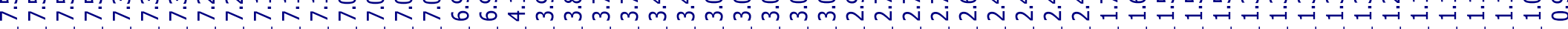

${ }^{1} \mathrm{H} N M R$ of $1 \mathrm{i}$ in $\mathrm{CDCl}_{3}$
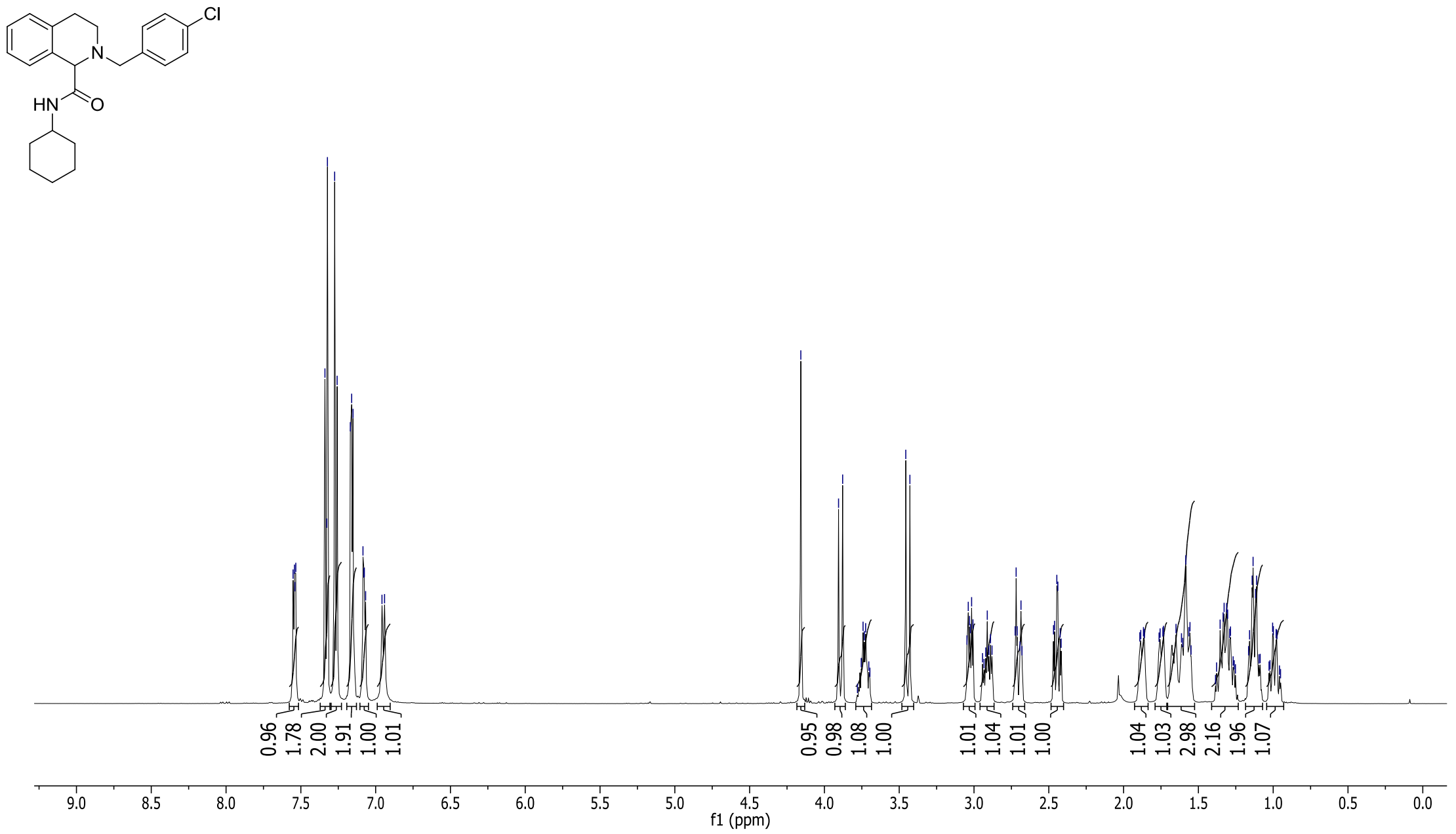


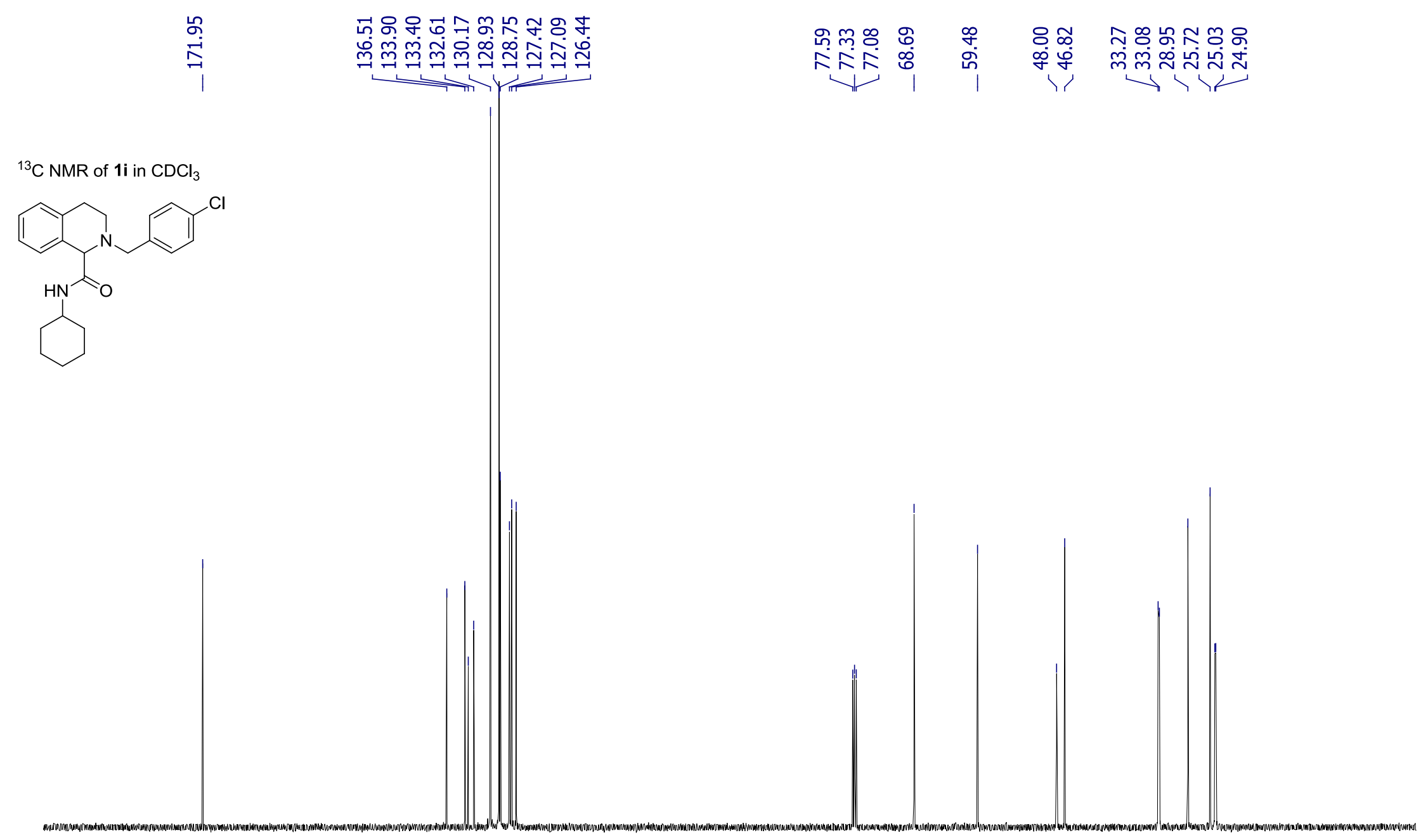

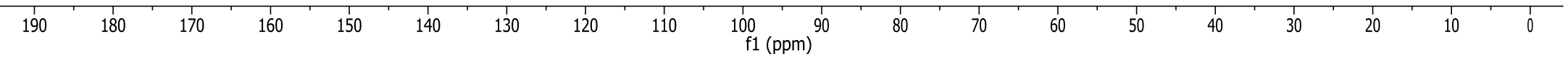




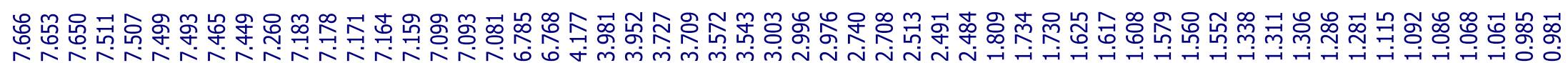

${ }^{1} \mathrm{H} \mathrm{NMR}$ of $1 \mathrm{j}$ in $\mathrm{CDCl}_{3}$
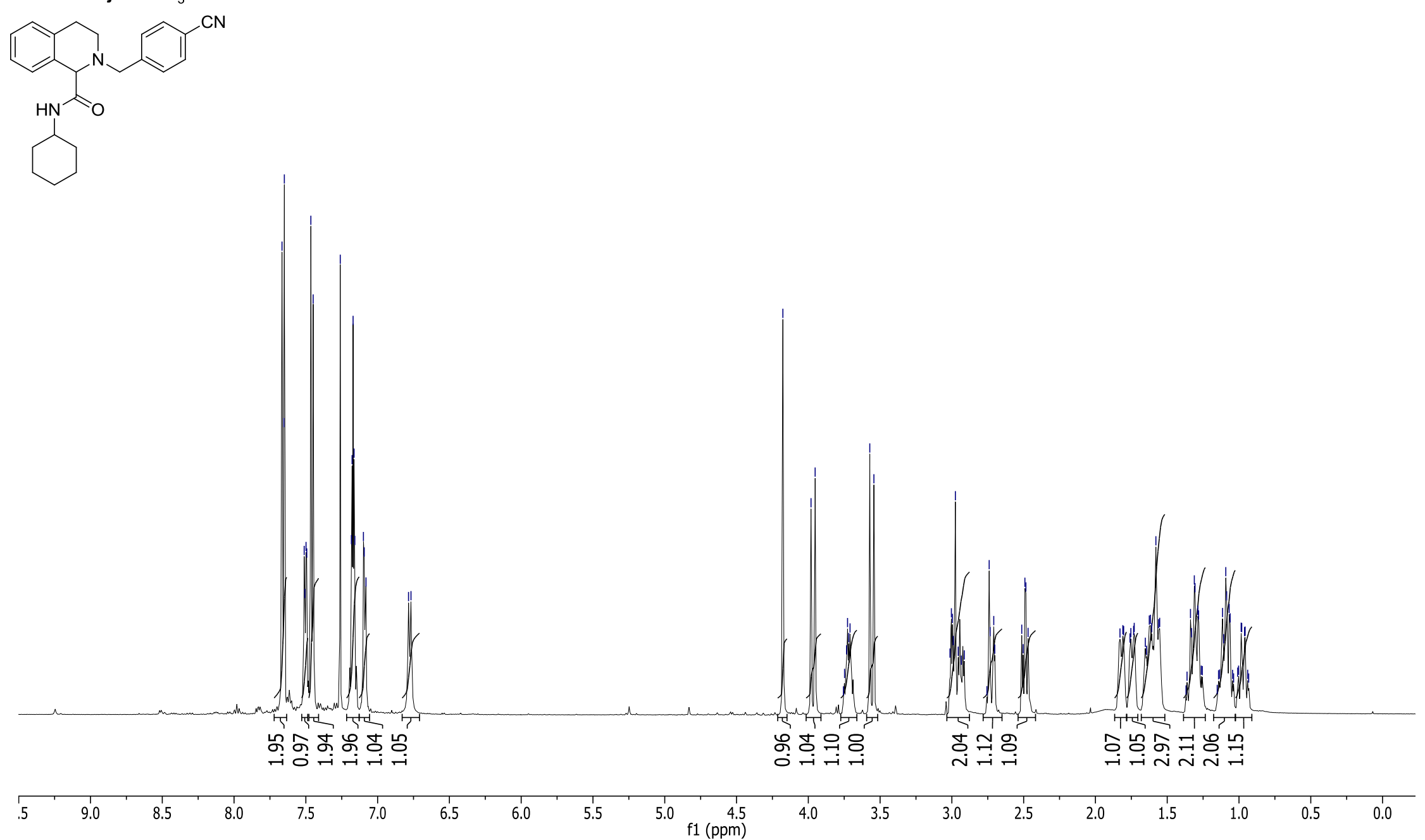
${ }^{13} \mathrm{C} \mathrm{NMR}$ of $1 \mathrm{j}$ in $\mathrm{CDCl}_{3}$

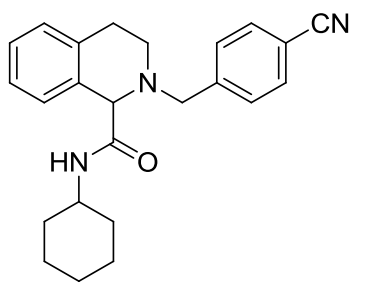

।
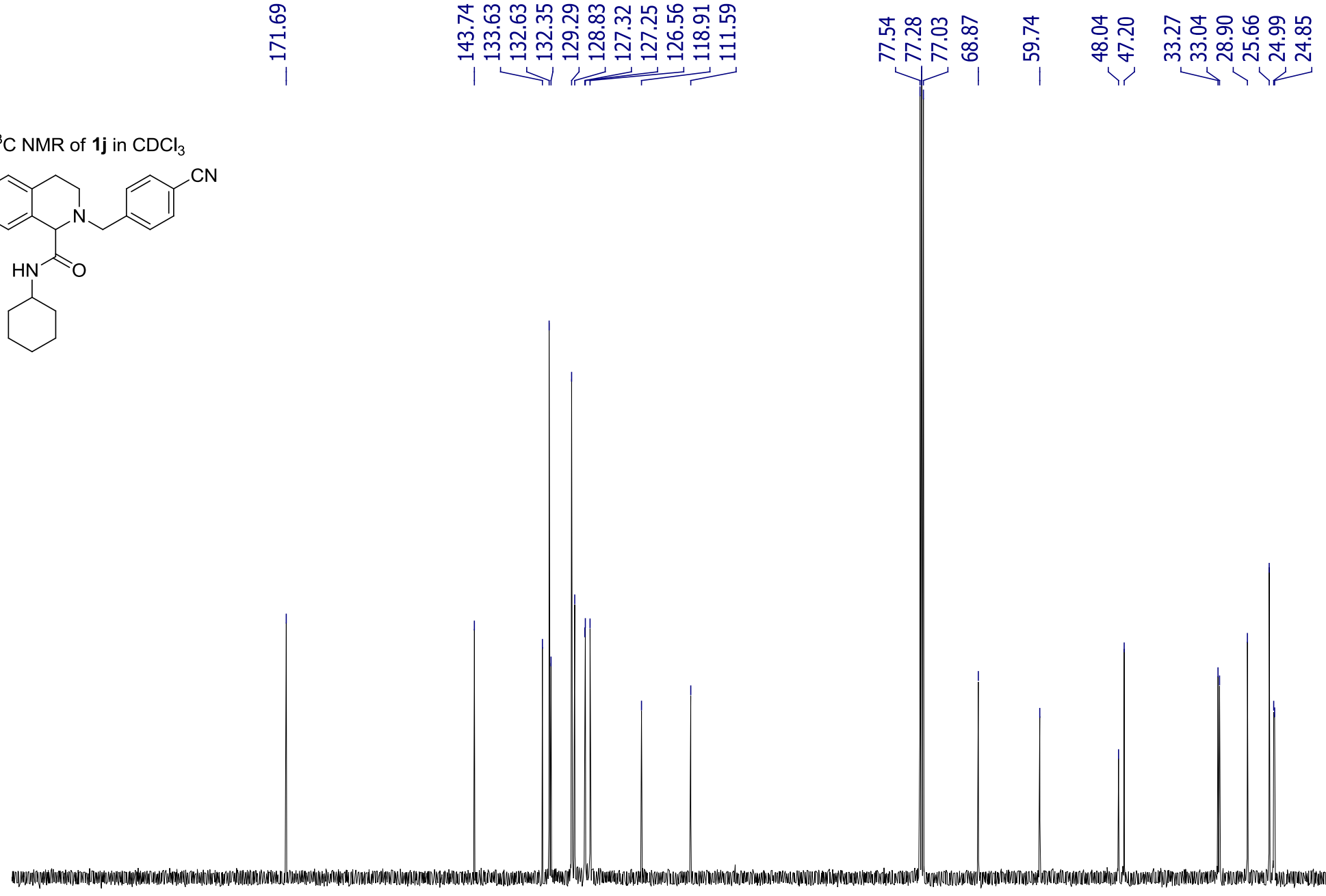

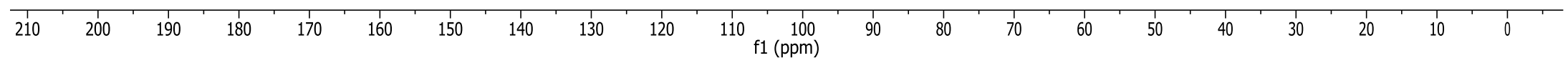




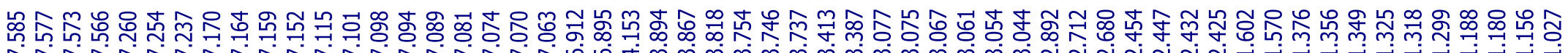

${ }^{1} \mathrm{H}$ NMR of $1 \mathbf{k}$ in $\mathrm{CDCl}_{3}$
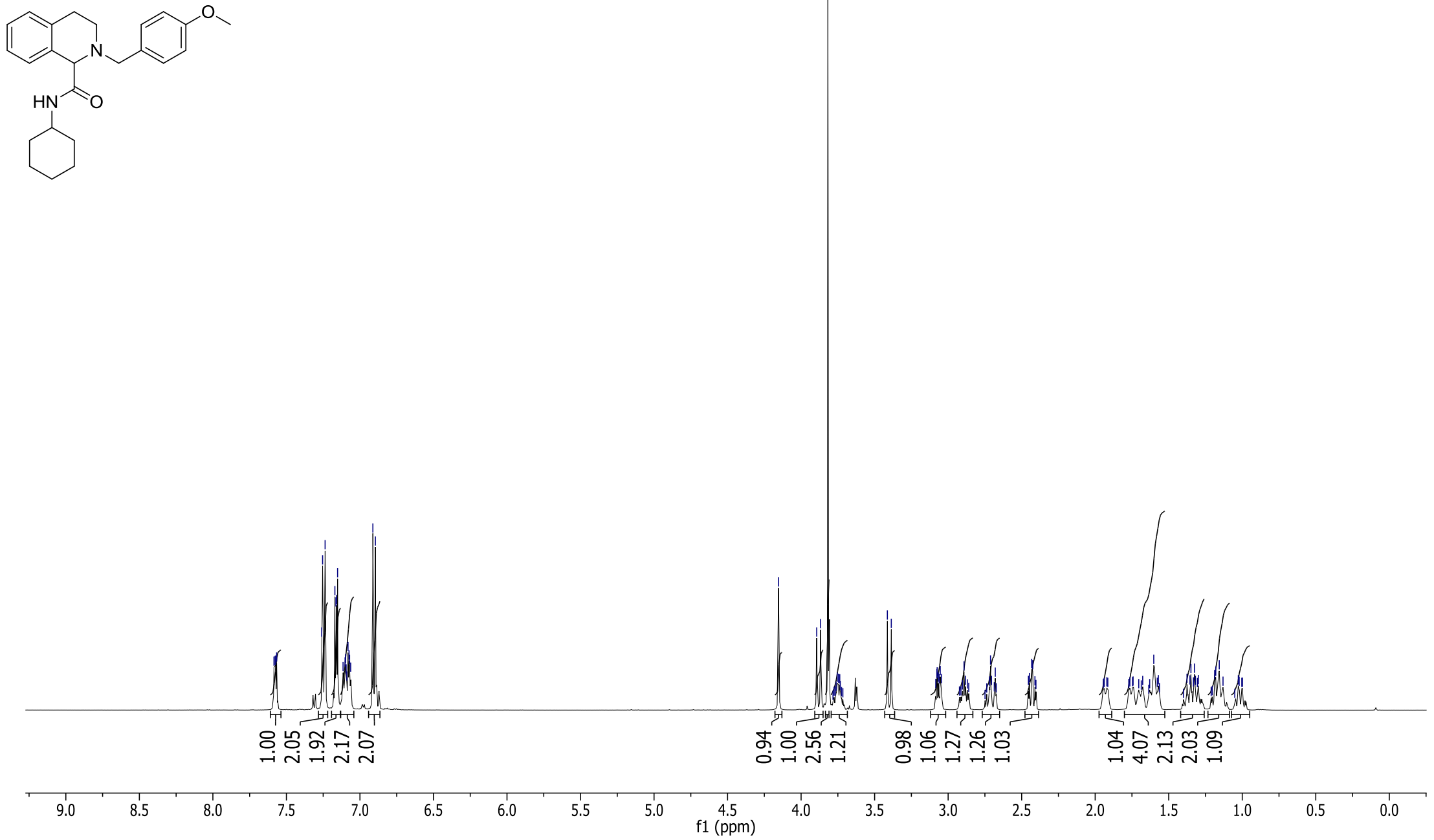


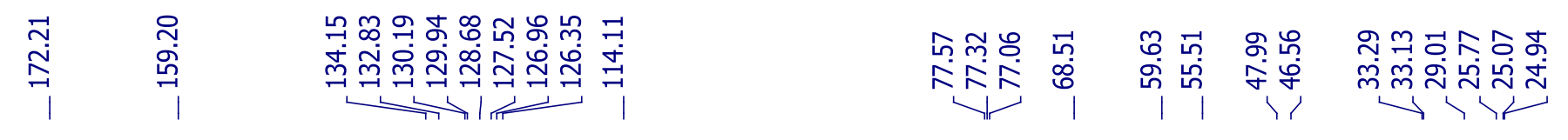

${ }^{13} \mathrm{C} \mathrm{NMR}$ of $1 \mathbf{k}$ in $\mathrm{CDCl}_{3}$
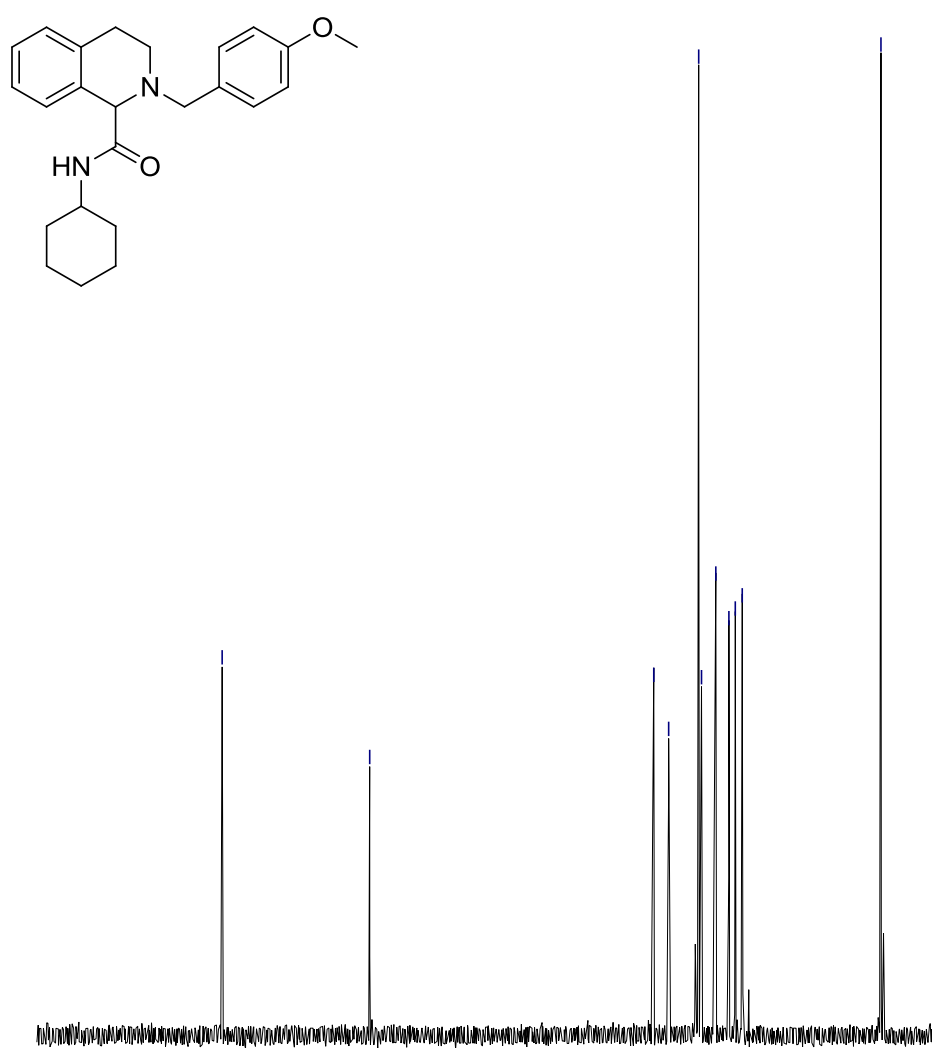

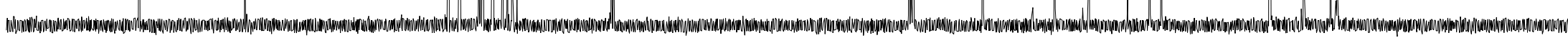

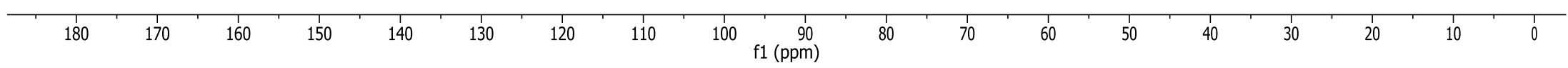




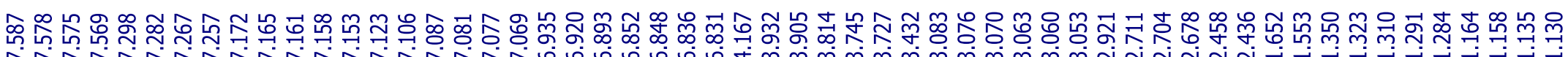

${ }^{1} \mathrm{H}$ NMR of 11 in $\mathrm{CDCl}_{3}$
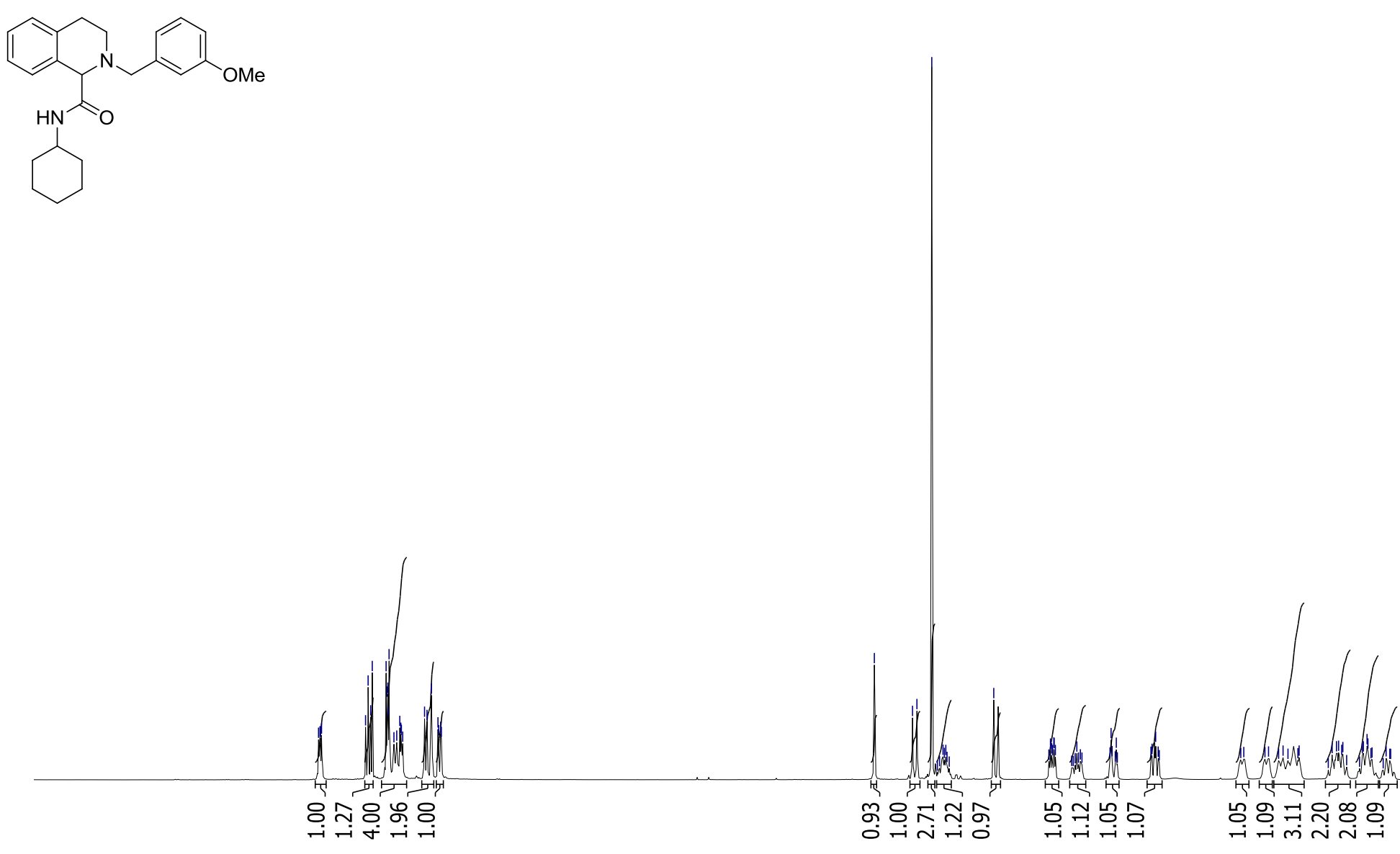

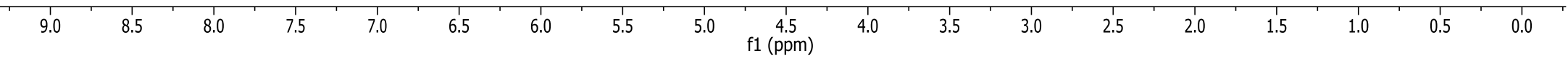




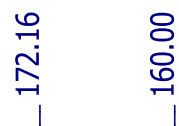

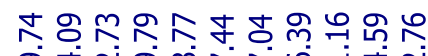

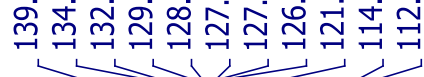

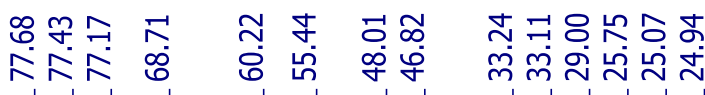

${ }^{13} \mathrm{C} \mathrm{NMR}$ of $1 \mathrm{l}$ in $\mathrm{CDCl}_{3}$
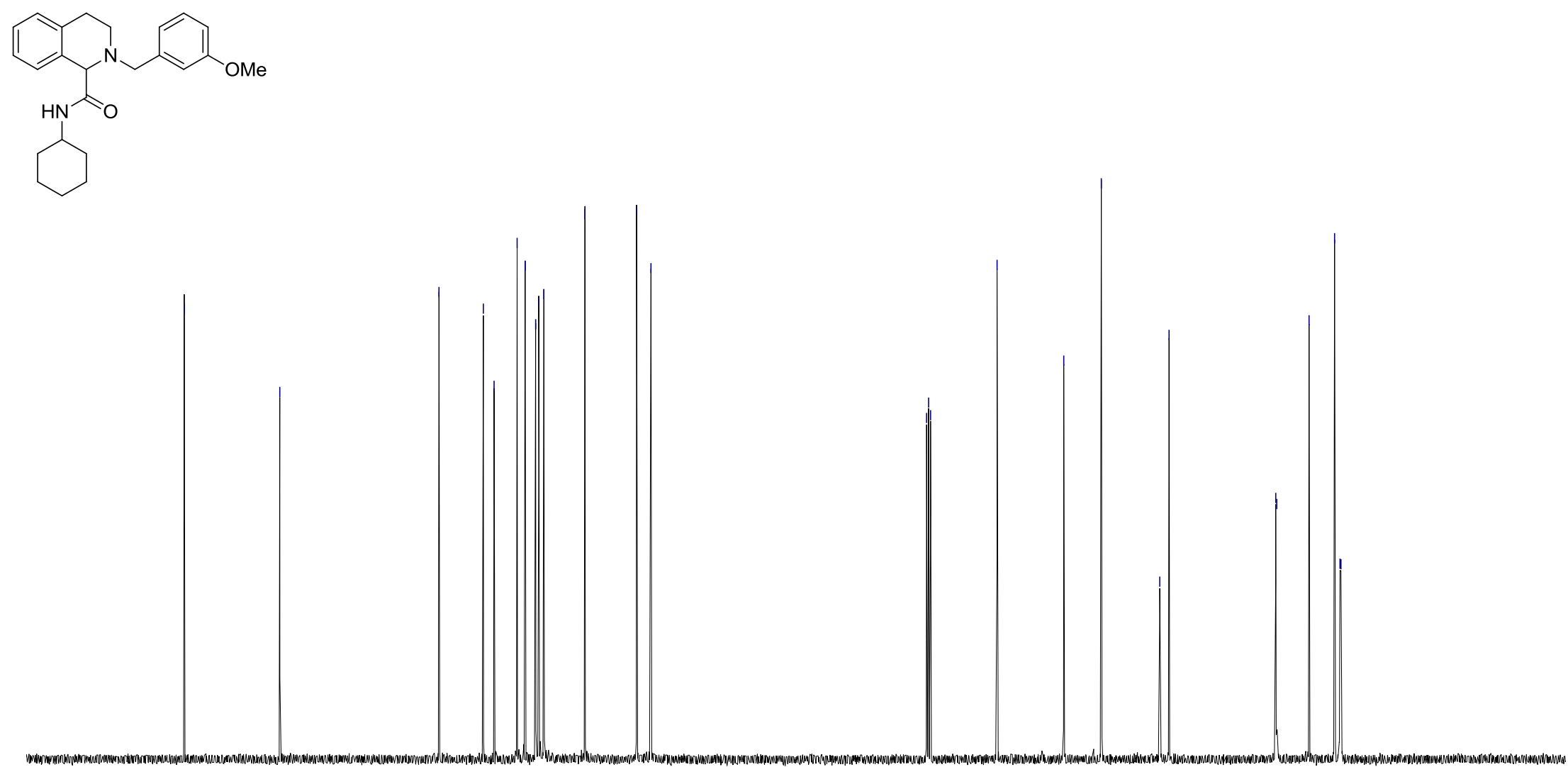

190
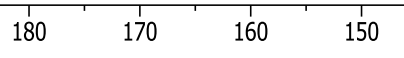

$140 \quad 130$

$120 \quad 110$

$100 \quad 90$

80

70

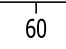

50

40

30

20 


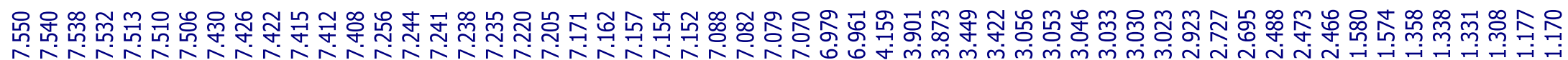

${ }^{1} \mathrm{H}$ NMR of $1 \mathrm{~m}$ in $\mathrm{CDCl}_{3}$
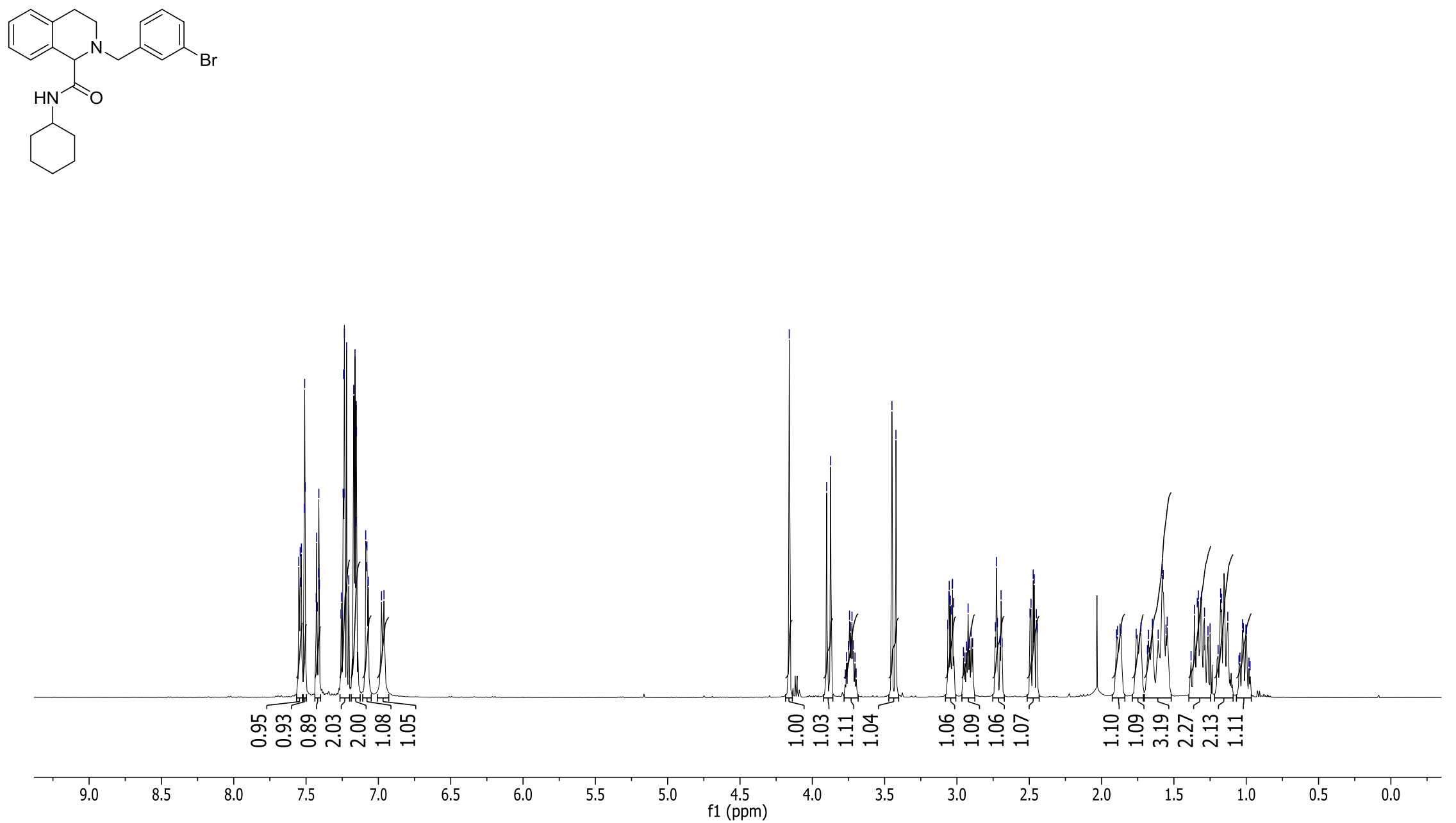


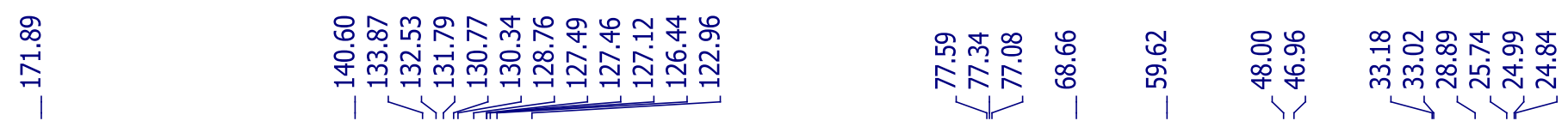

${ }^{13} \mathrm{C} \mathrm{NMR}$ of $1 \mathrm{~m}$ in $\mathrm{CDCl}_{3}$
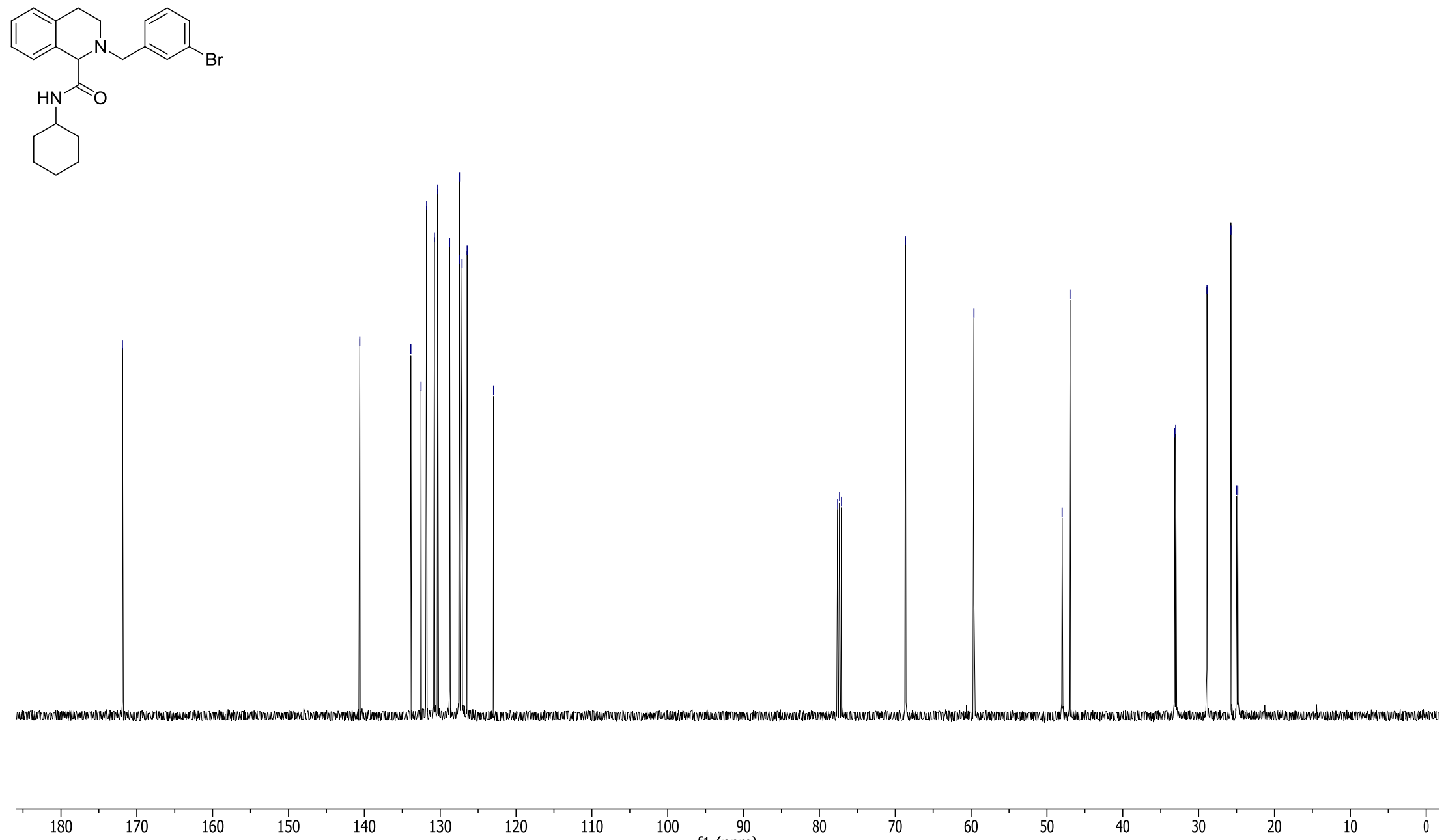

110

100

f1 $(\mathrm{ppm})$

80

70

60

50

40

20 


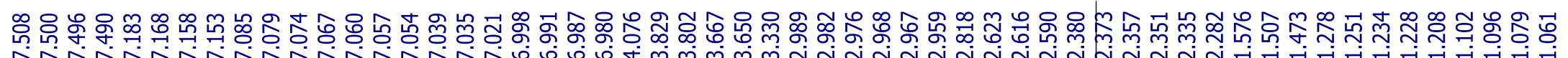
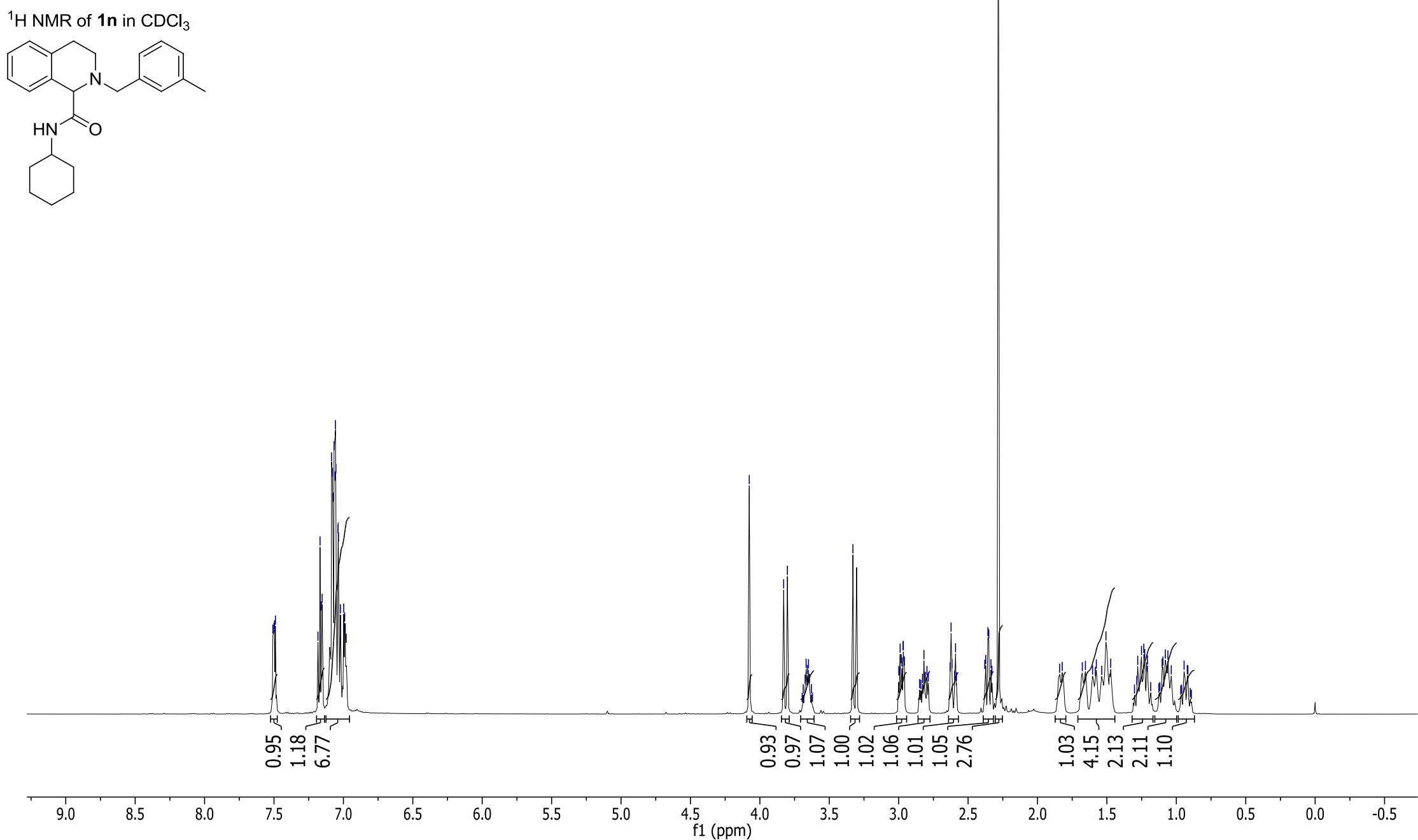


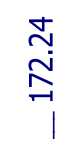

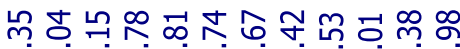

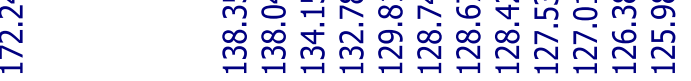

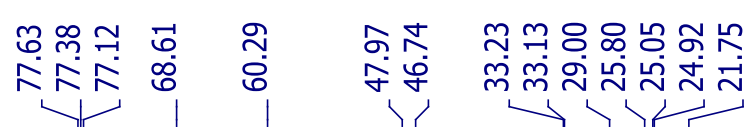

${ }^{13} \mathrm{C} \mathrm{NMR}$ of $1 \mathrm{n}$ in $\mathrm{CDCl}_{3}$
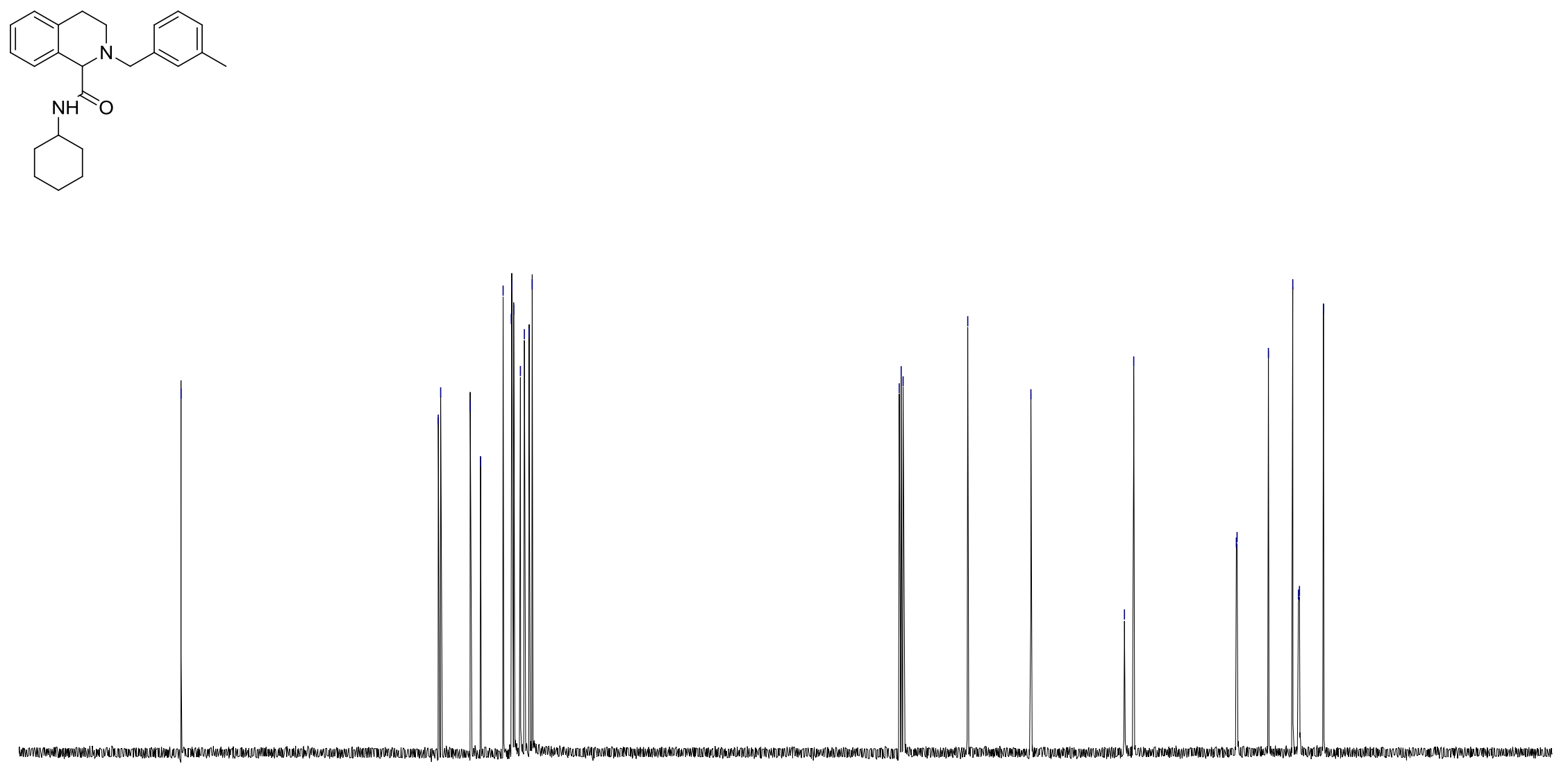

190

$180 \quad 170$

160

150

140

130

120

110

$100 \quad 90$

S-43 


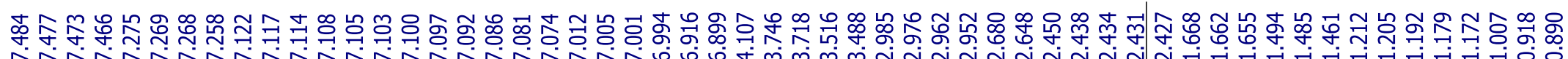

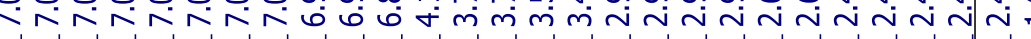
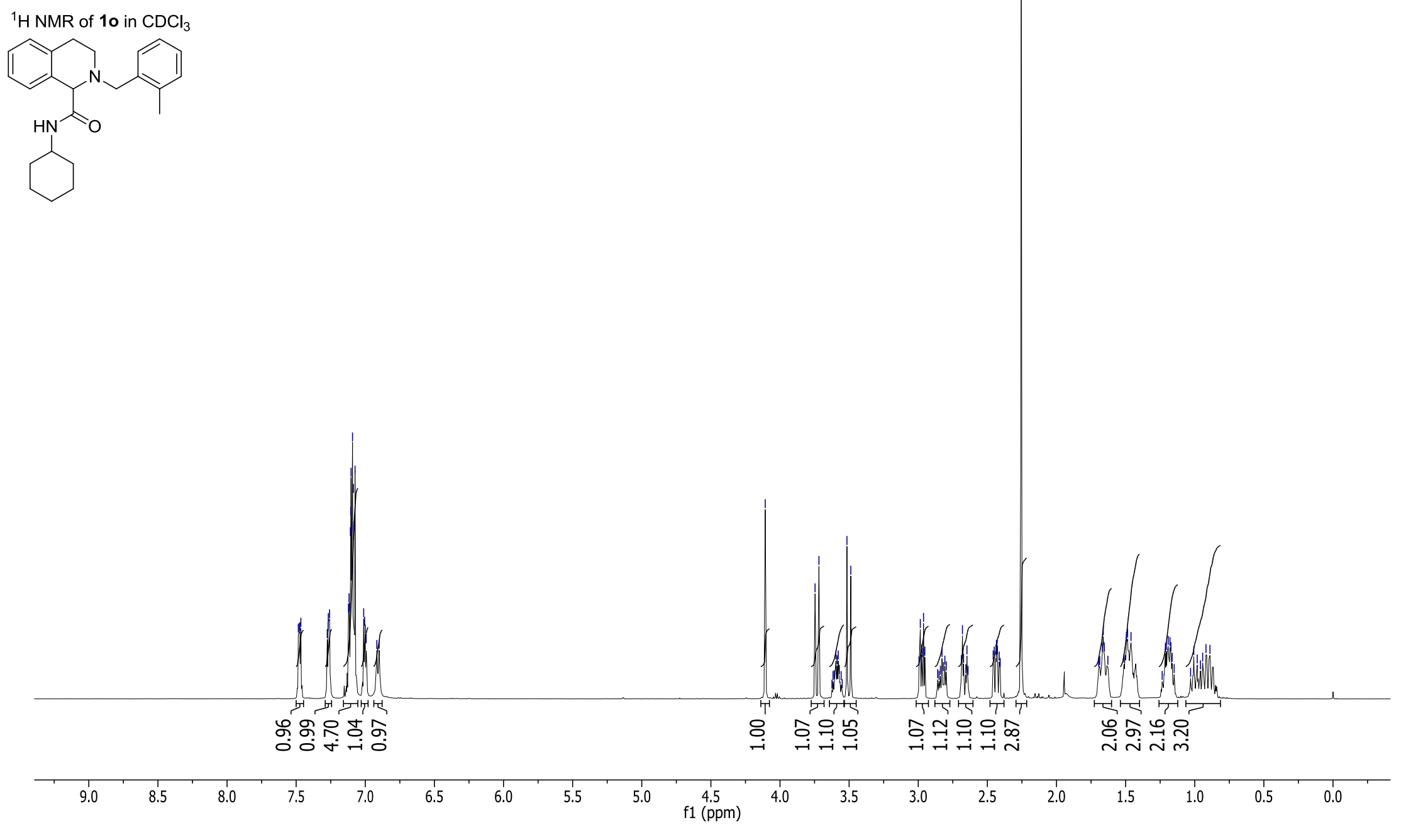

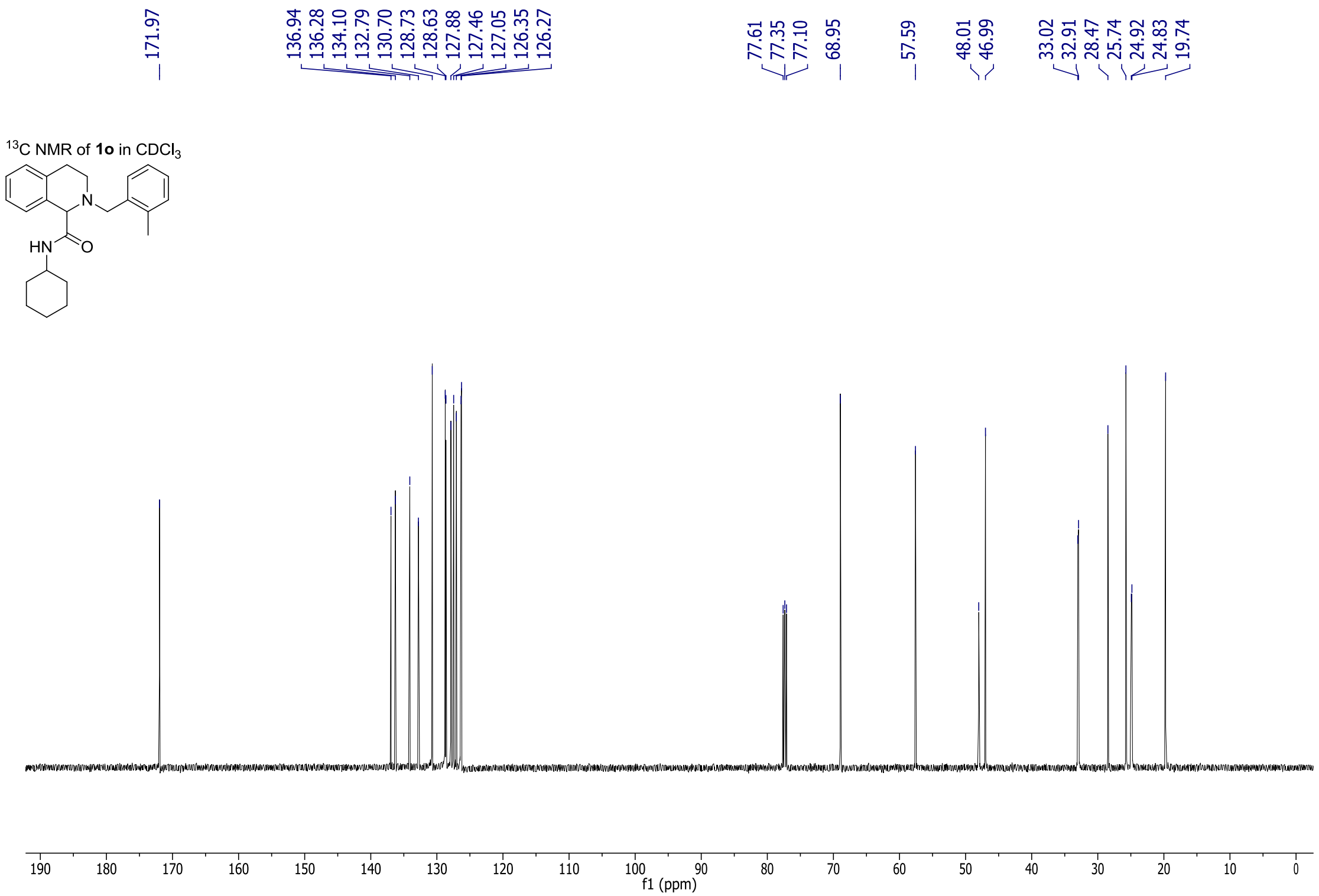


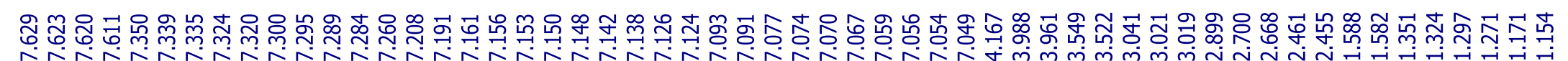

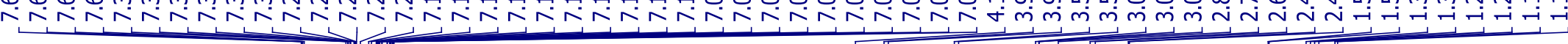

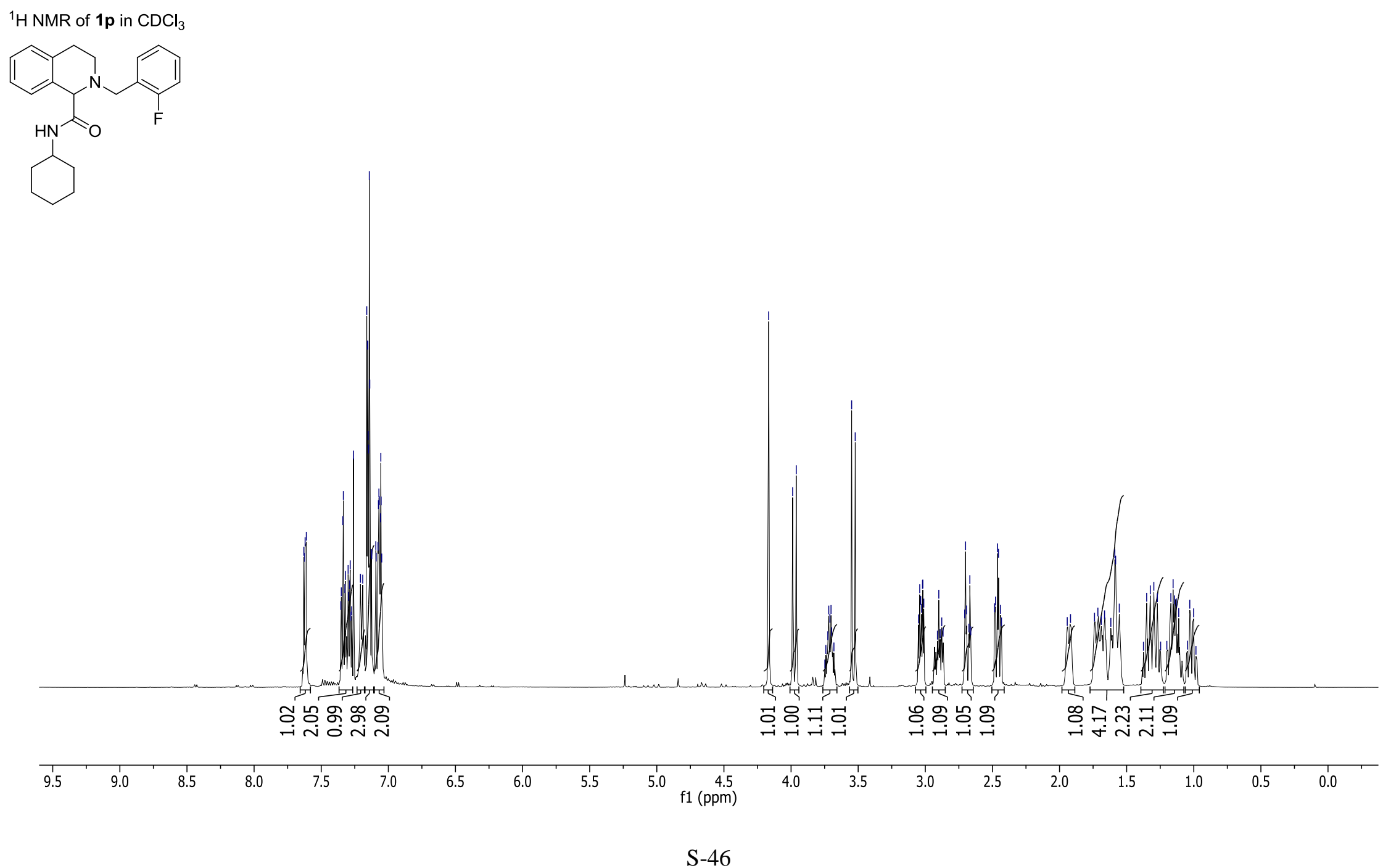



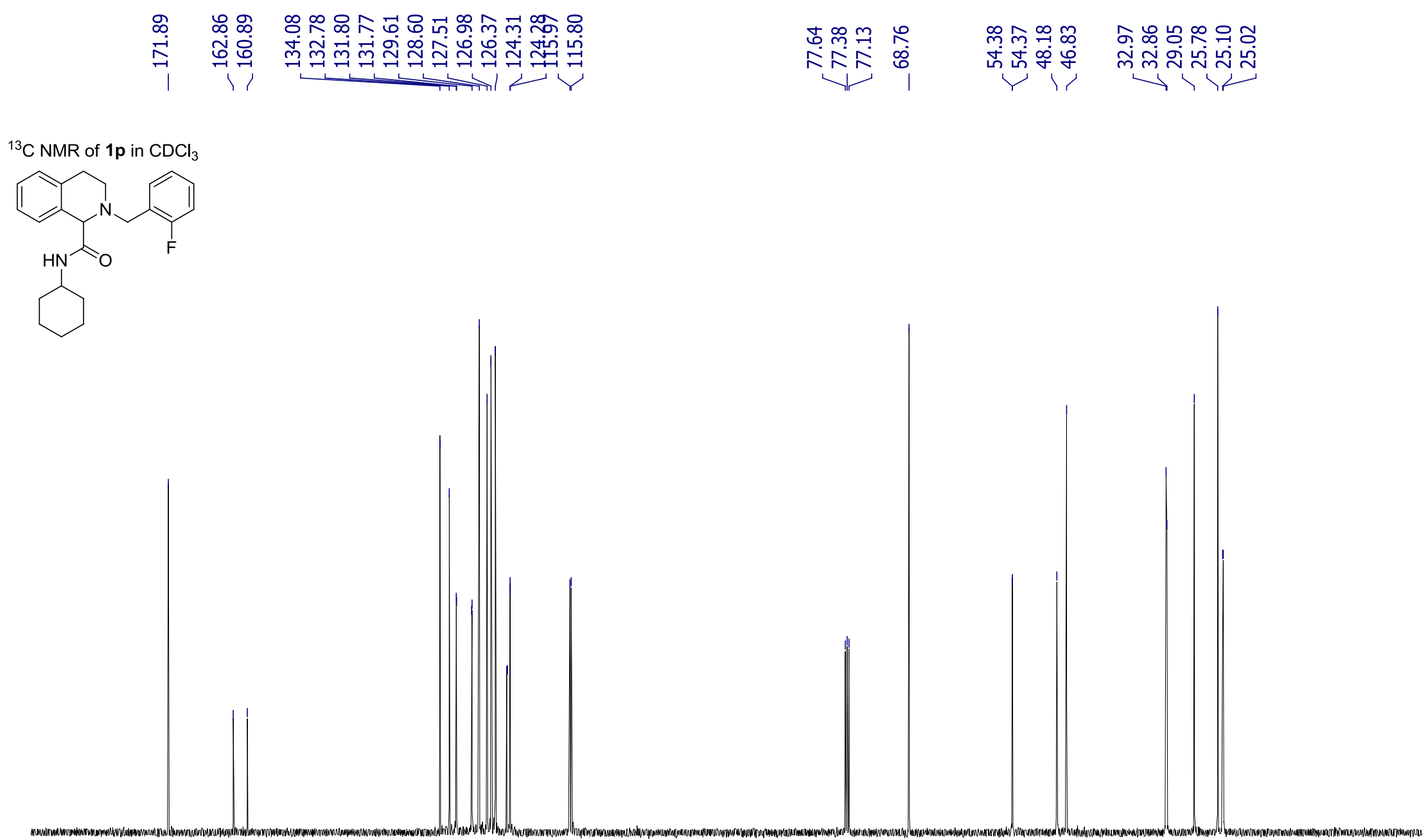

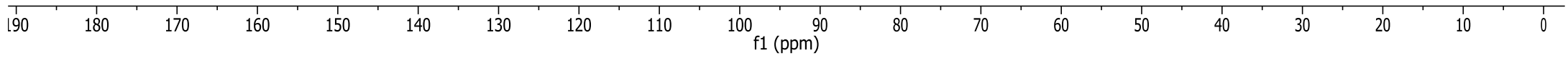




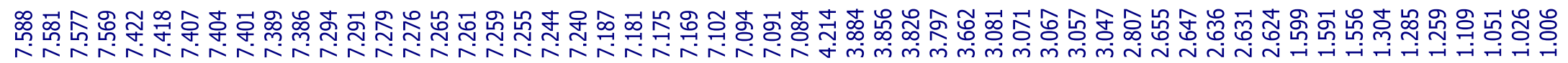

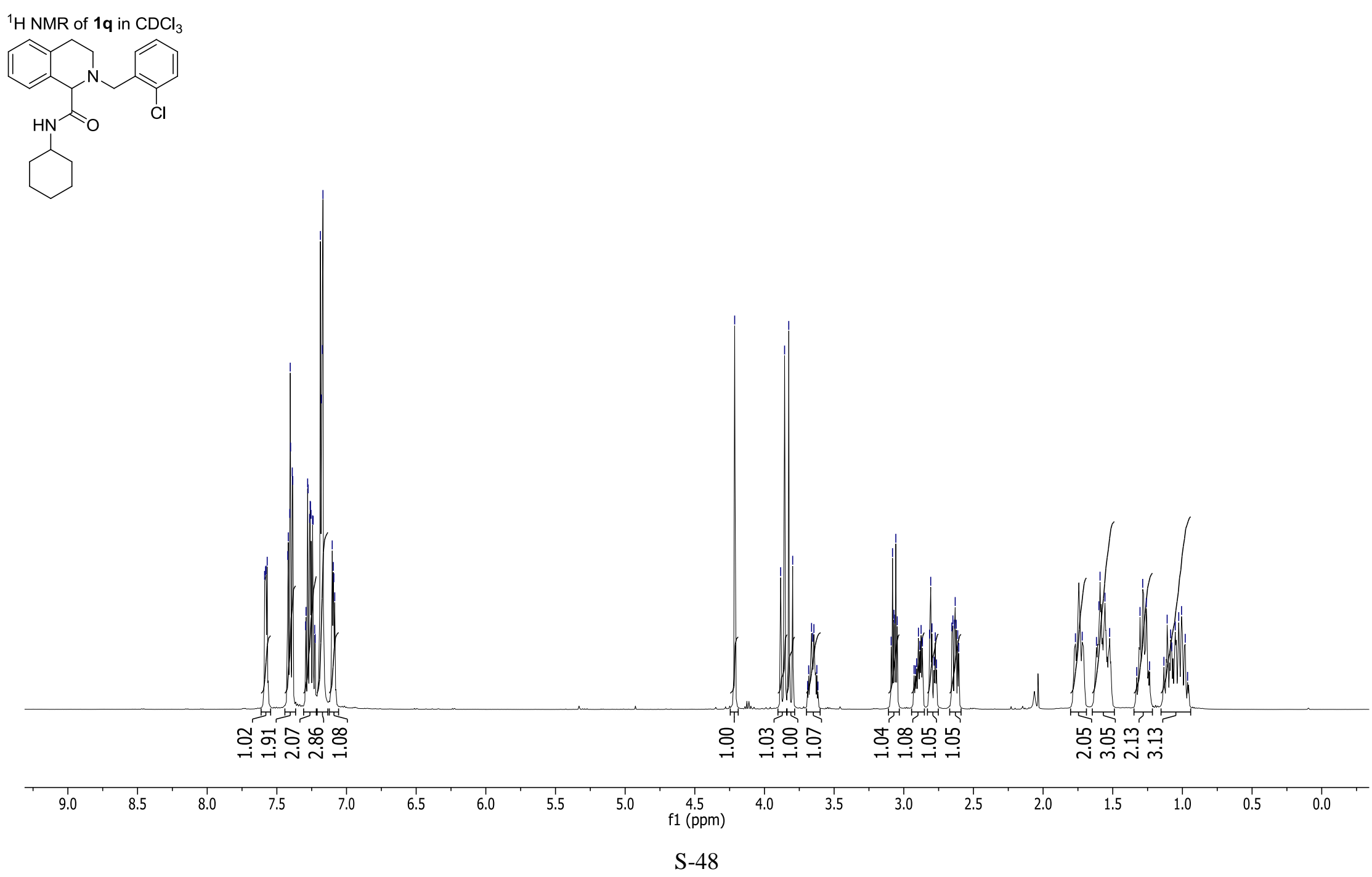


${ }^{13} \mathrm{C} \mathrm{NMR} \mathrm{of} 1 \mathrm{q}$ in $\mathrm{CDCl}_{3}$
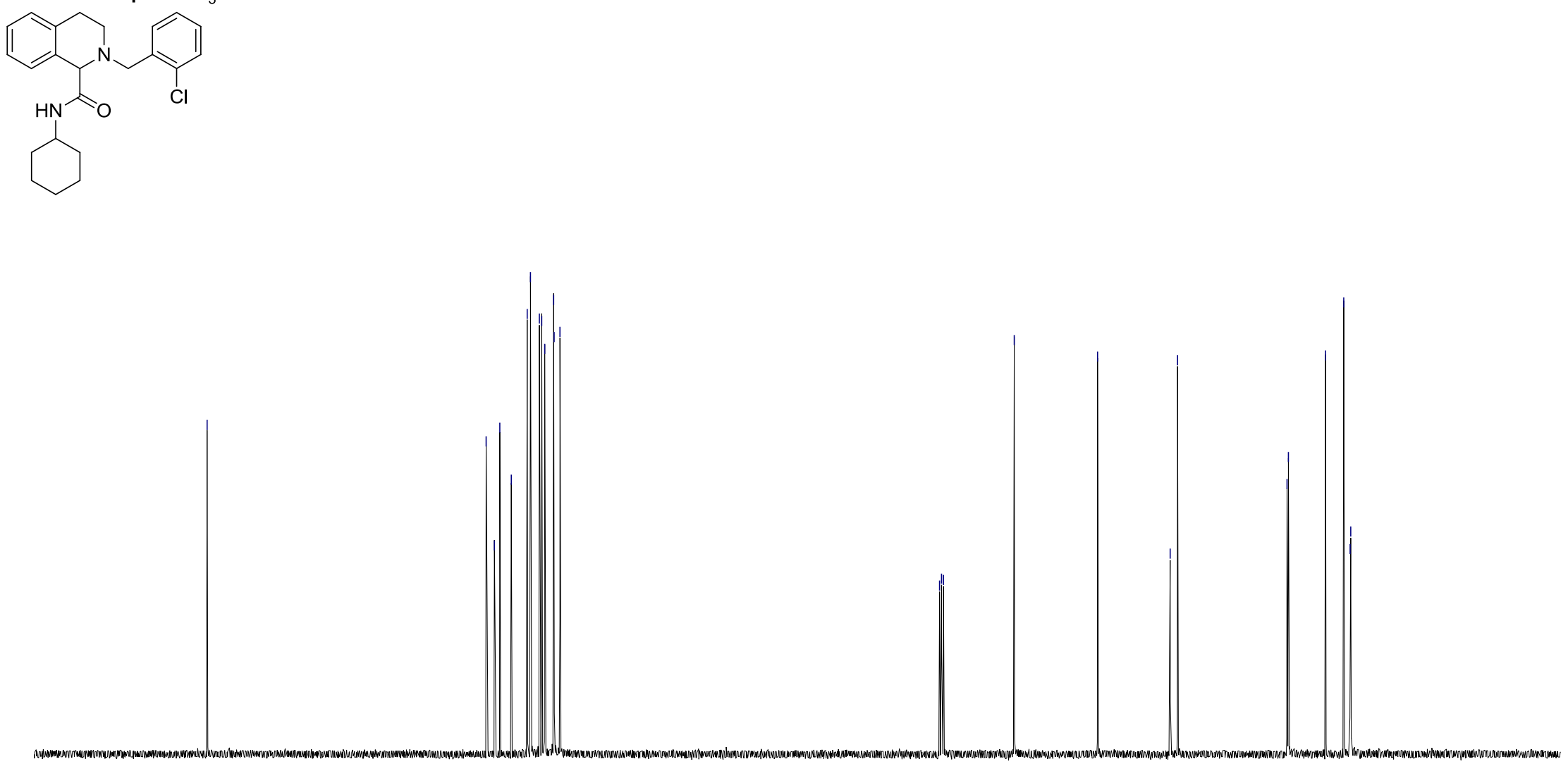

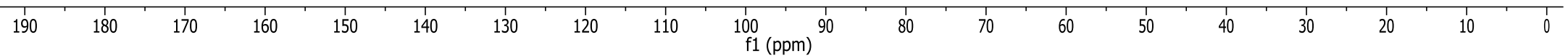




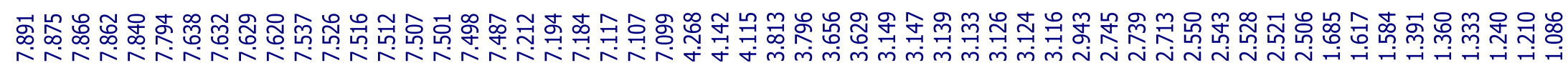

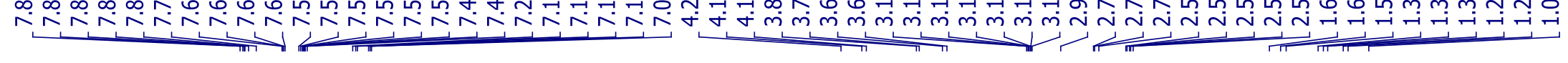
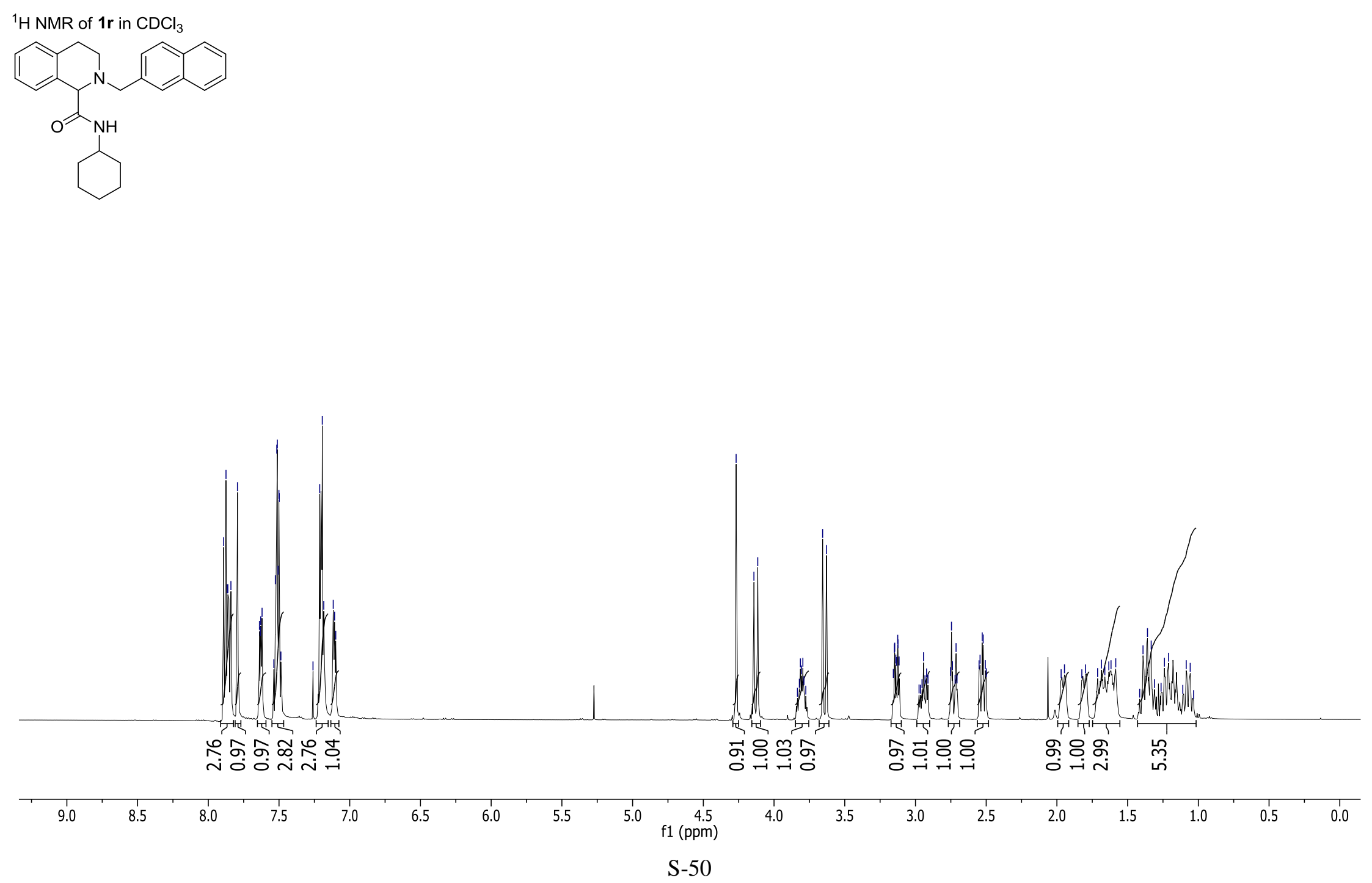


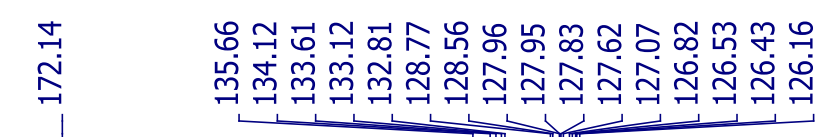

${ }^{13} \mathrm{C} \mathrm{NMR}$ of $1 \mathbf{r}$ in $\mathrm{CDCl}_{3}$

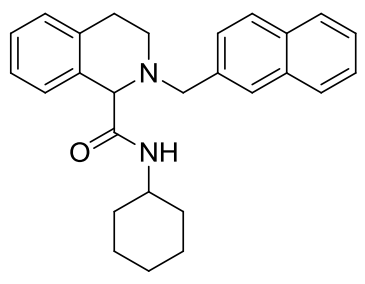

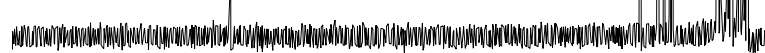

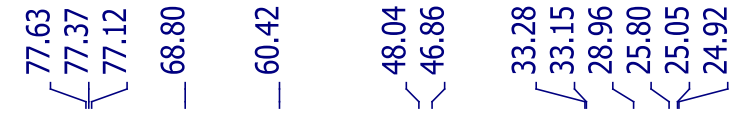

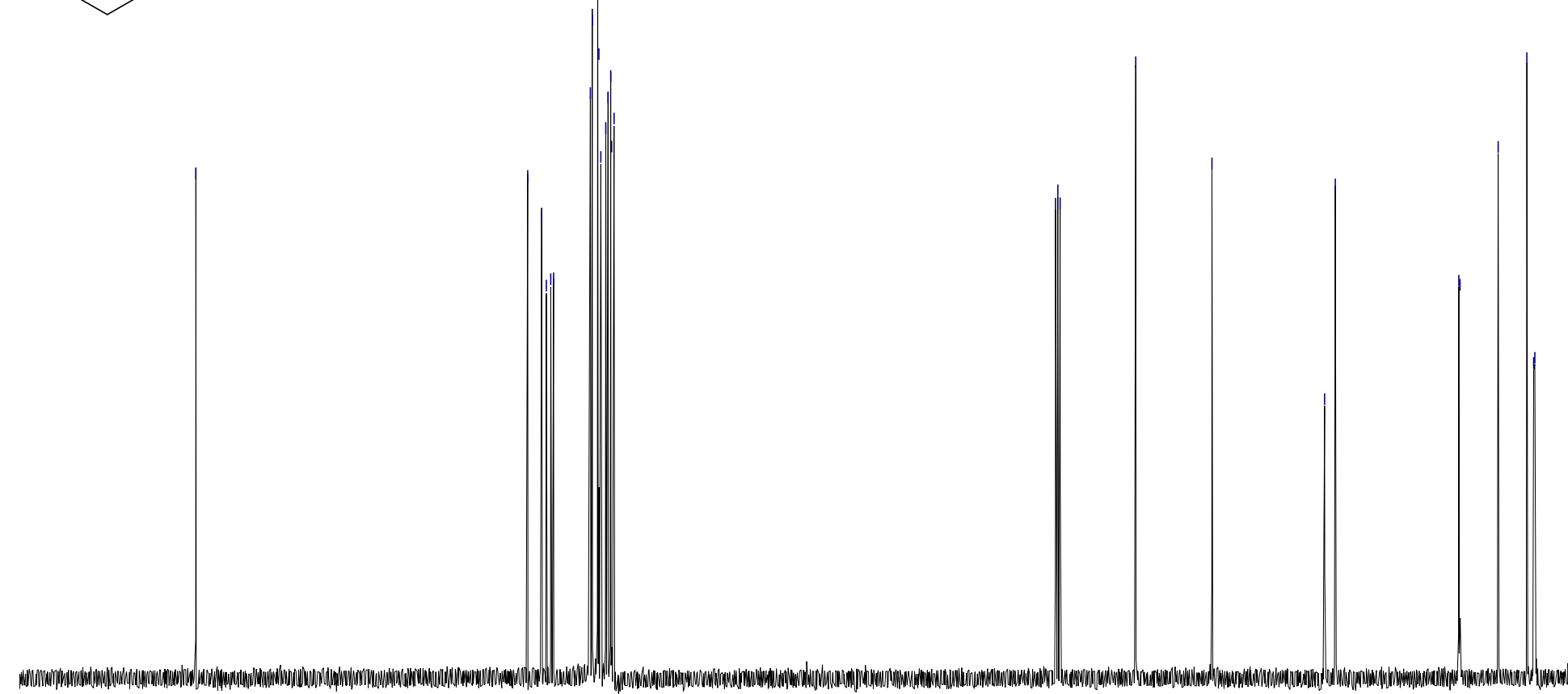

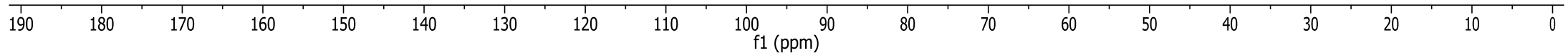




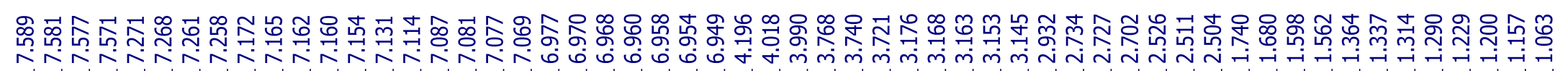<smiles>CC(C)(C)OCCNCCc1ccccc1CN(Cc1ccccc1)C(=O)NC1CCCCC1</smiles>

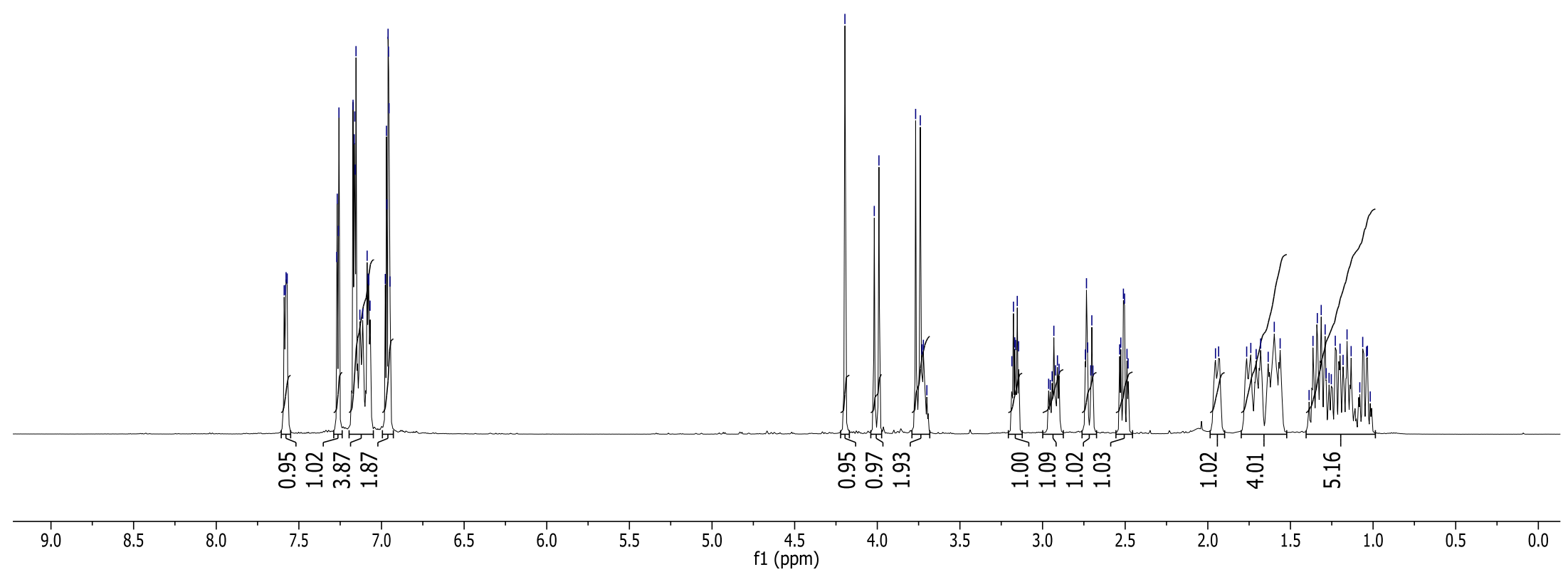




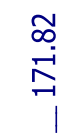

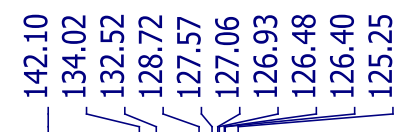

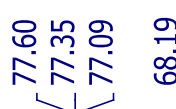

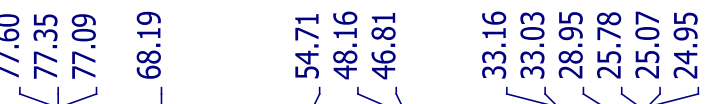

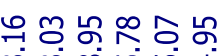

${ }^{13} \mathrm{C} \mathrm{NMR}$ of $1 \mathrm{~s}$ in $\mathrm{CDCl}_{3}$
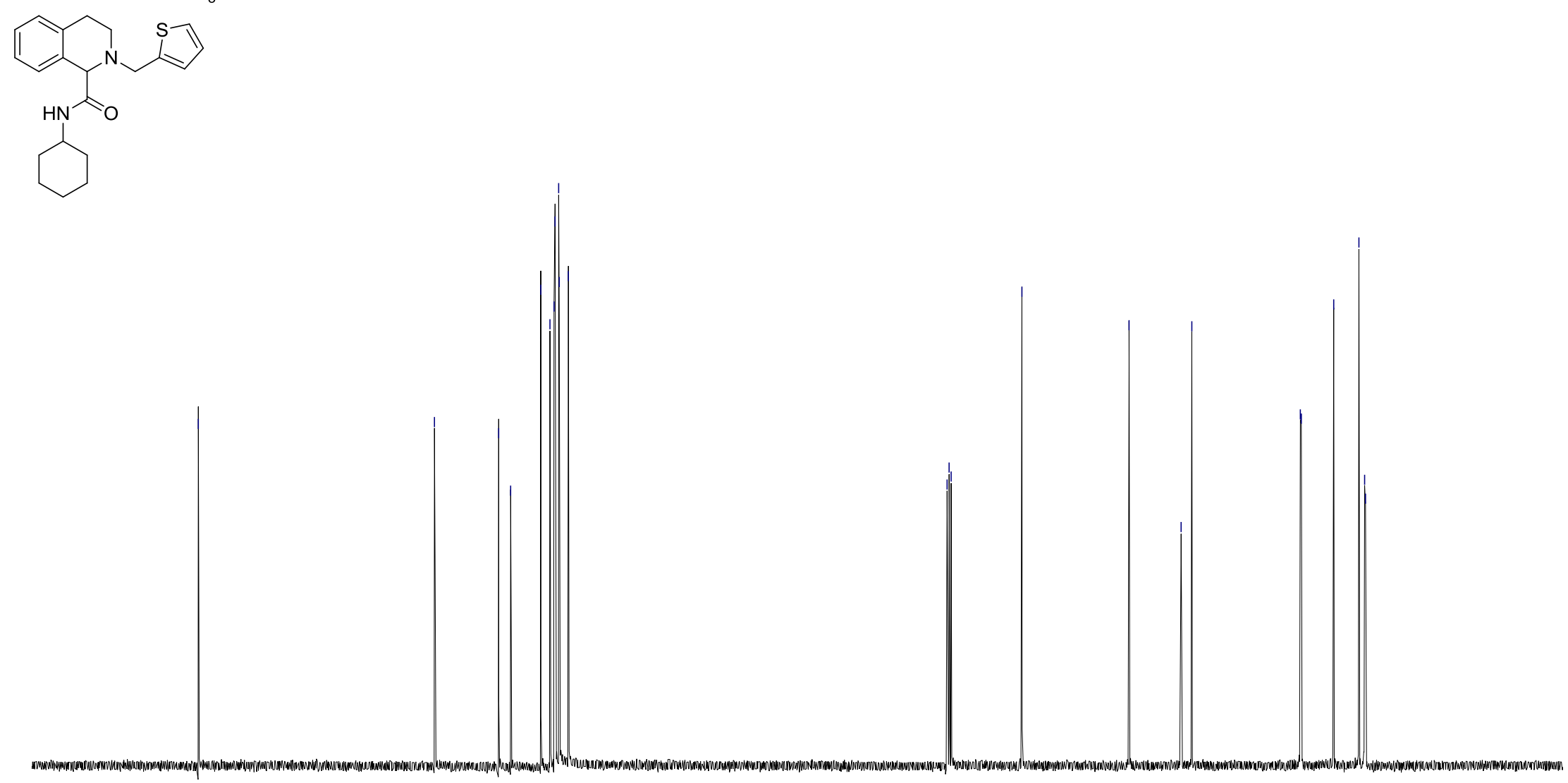

1
190

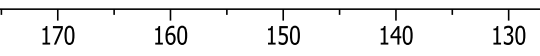

120

110

100 f1 (ppm)

$80 \quad 70$

60

50

40

$30 \quad 20$ 


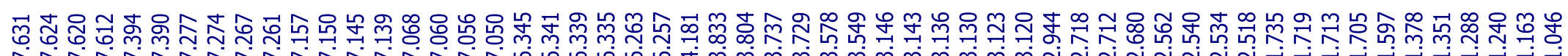

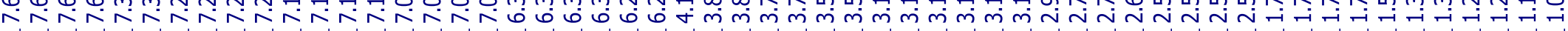

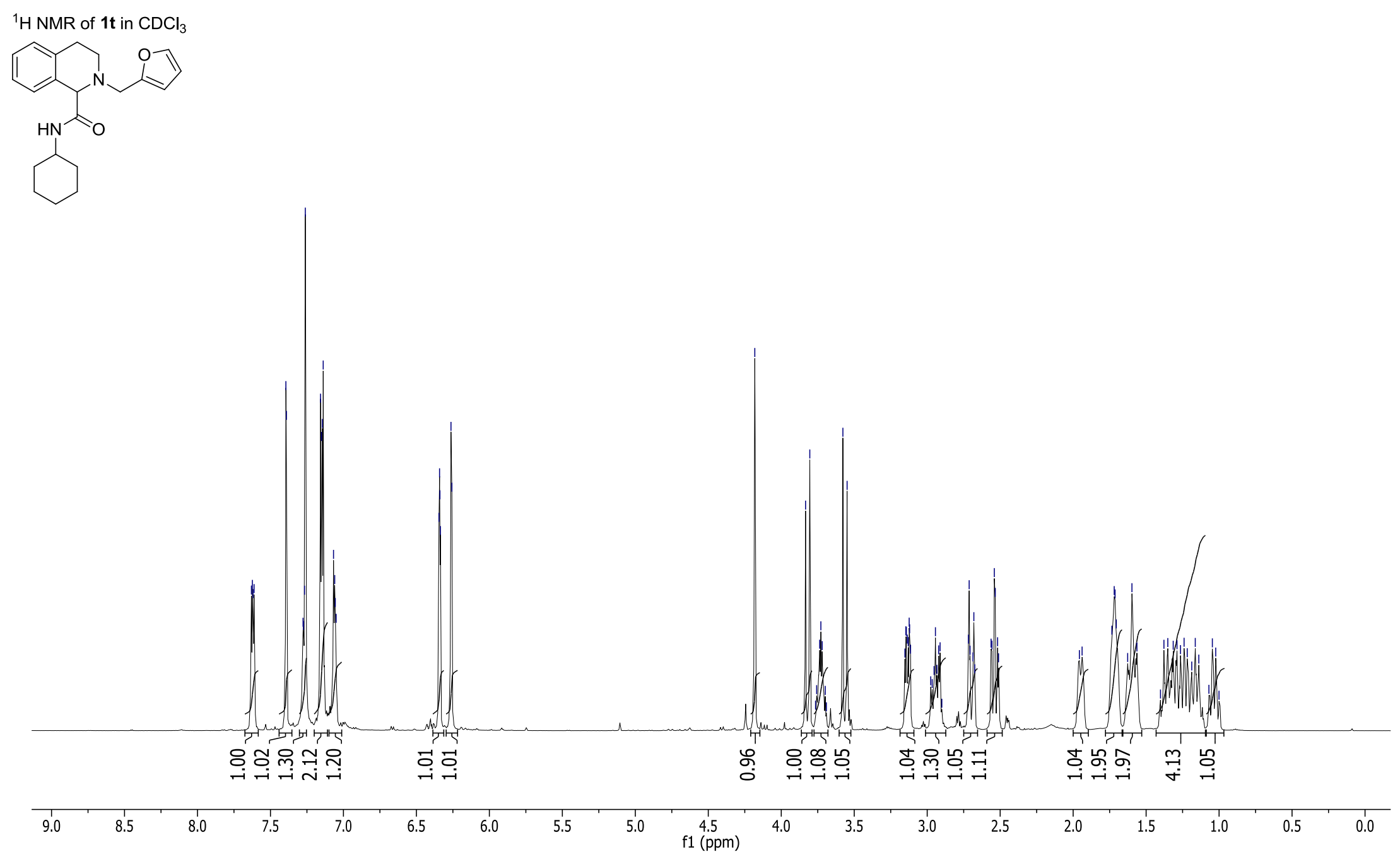




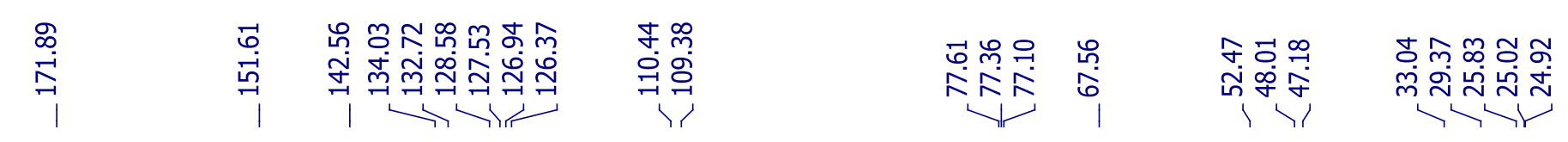

${ }^{13} \mathrm{C} \mathrm{NMR}$ of $1 \mathrm{t}$ in $\mathrm{CDCl}_{3}$
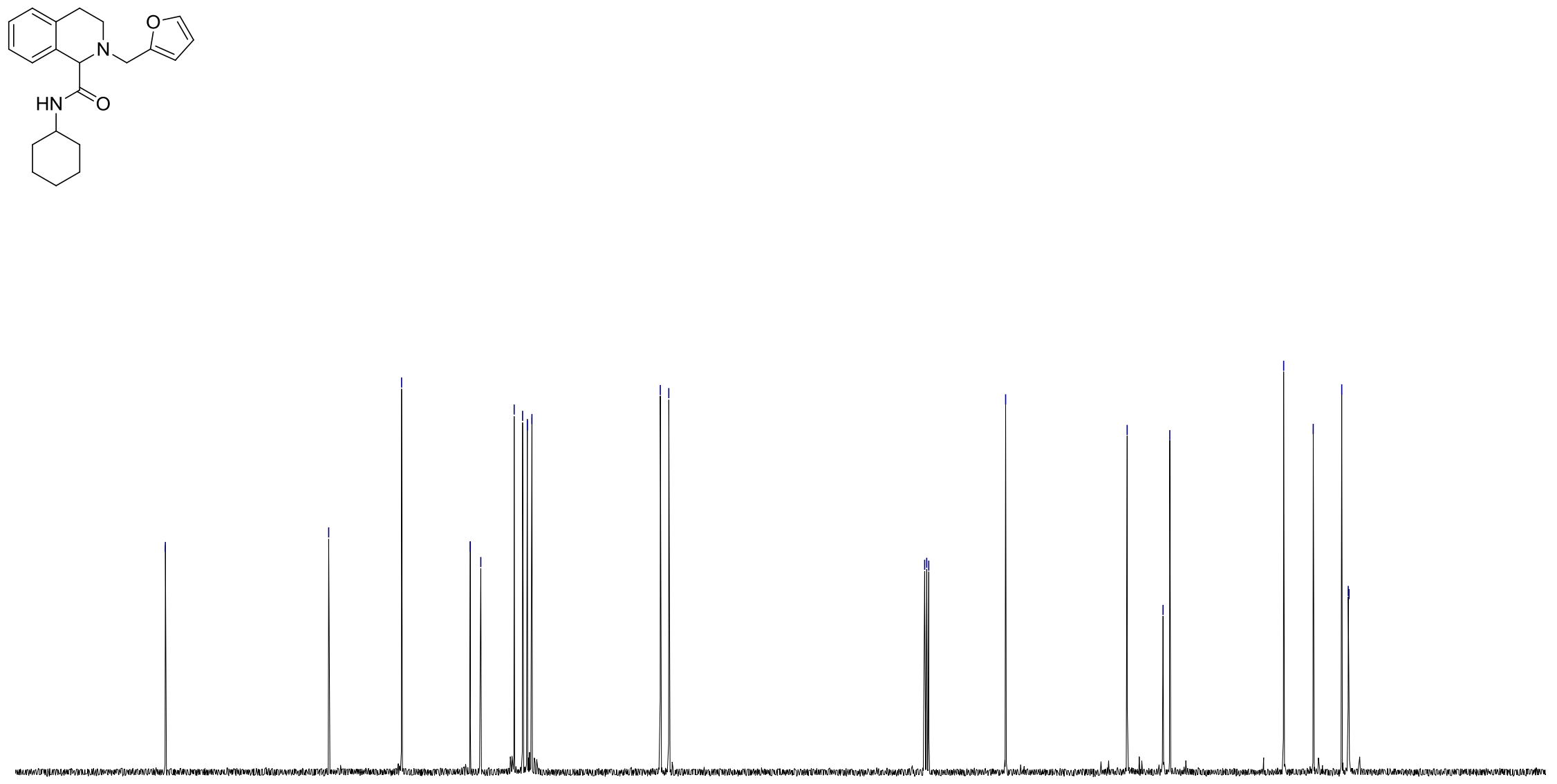

90

$180 \quad 170$

160

150

140

130

120

110 $100 \quad 90$
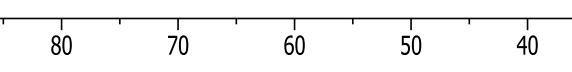


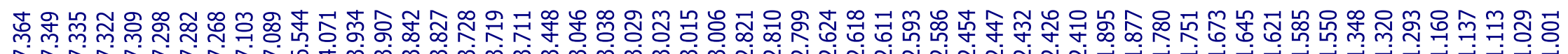

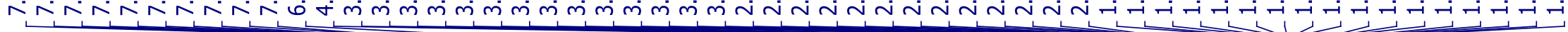

${ }^{1} \mathrm{H}$ NMR of $1 \mathrm{u}$ in $\mathrm{CDCl}_{3}$
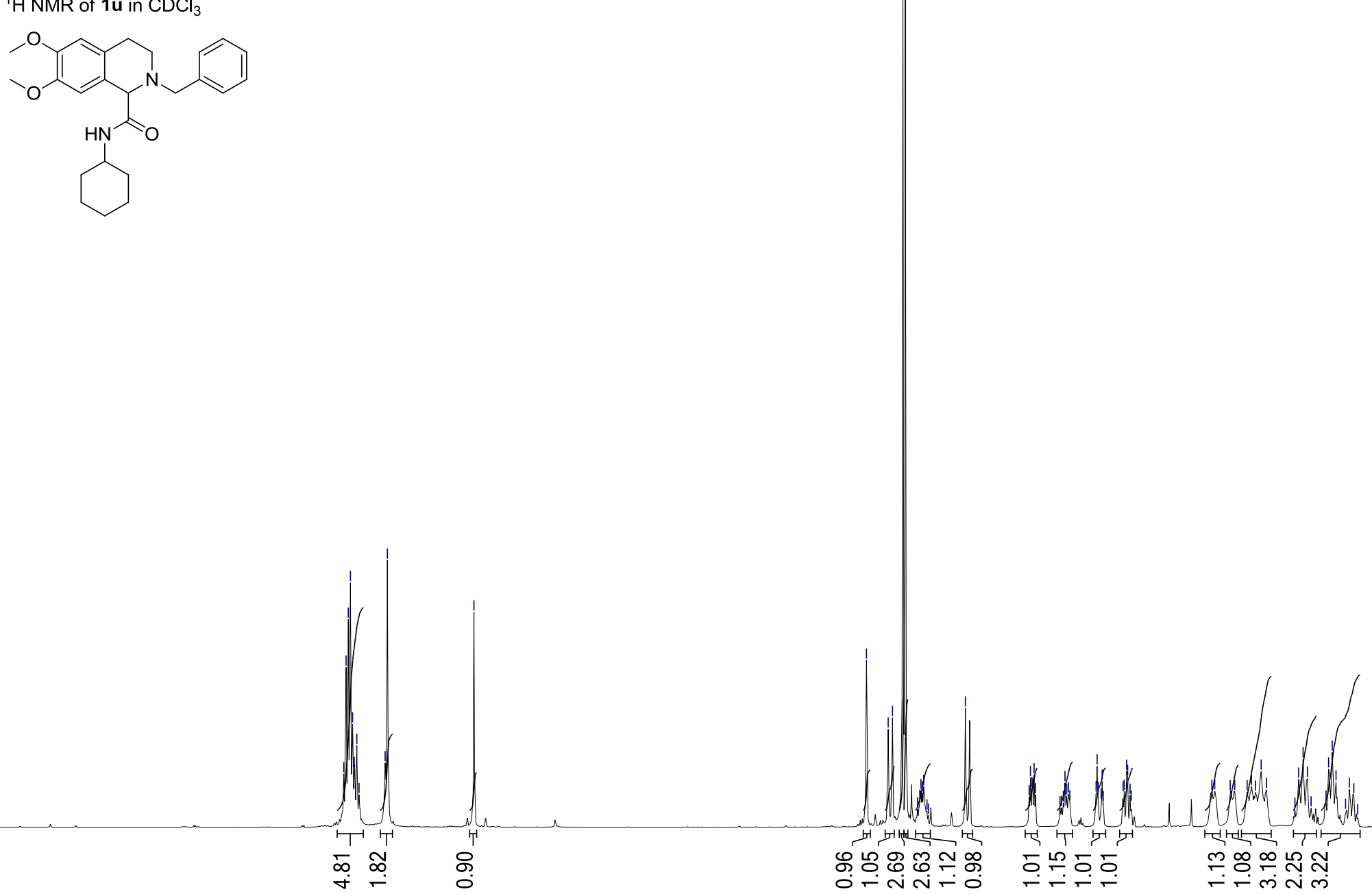


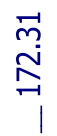

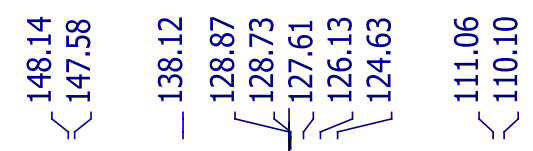

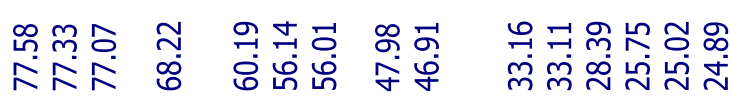

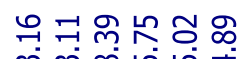

${ }^{13} \mathrm{C} \mathrm{NMR}$ of $1 \mathrm{u}$ in $\mathrm{CDCl}_{3}$<smiles>COc1cc2c(cc1OC)C(C(=O)NC1CCCCC1)N(Cc1ccccc1)CC2</smiles>

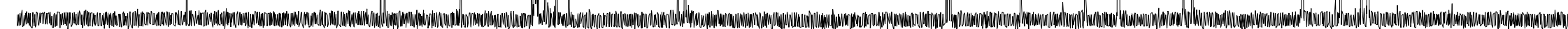




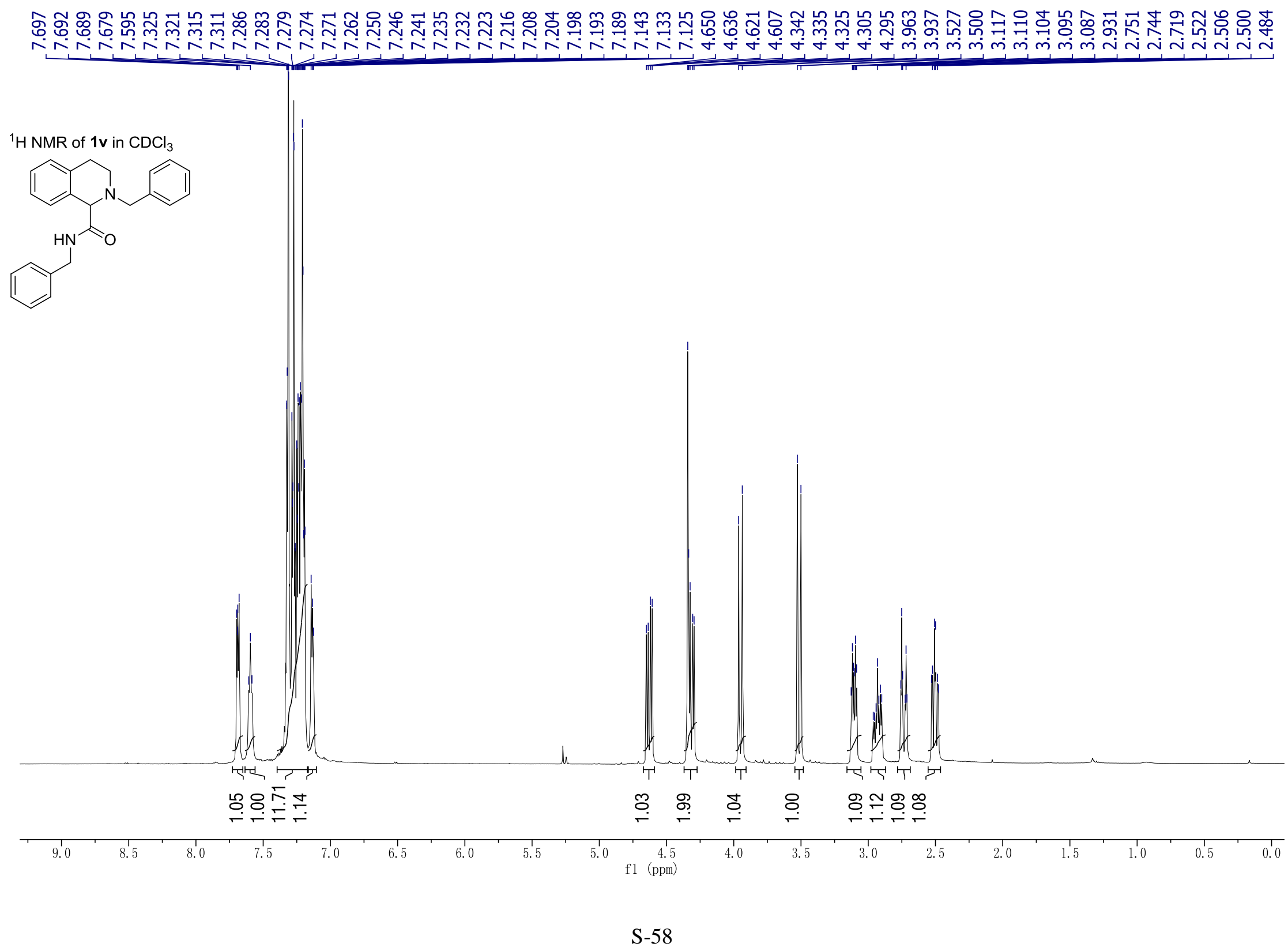




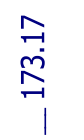

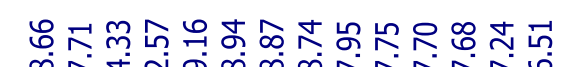

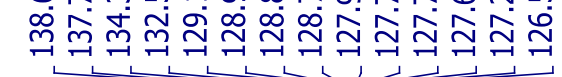

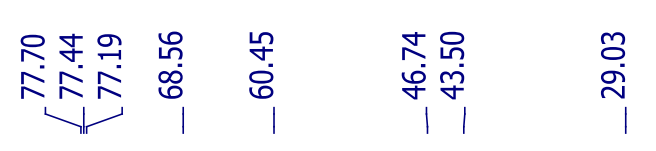

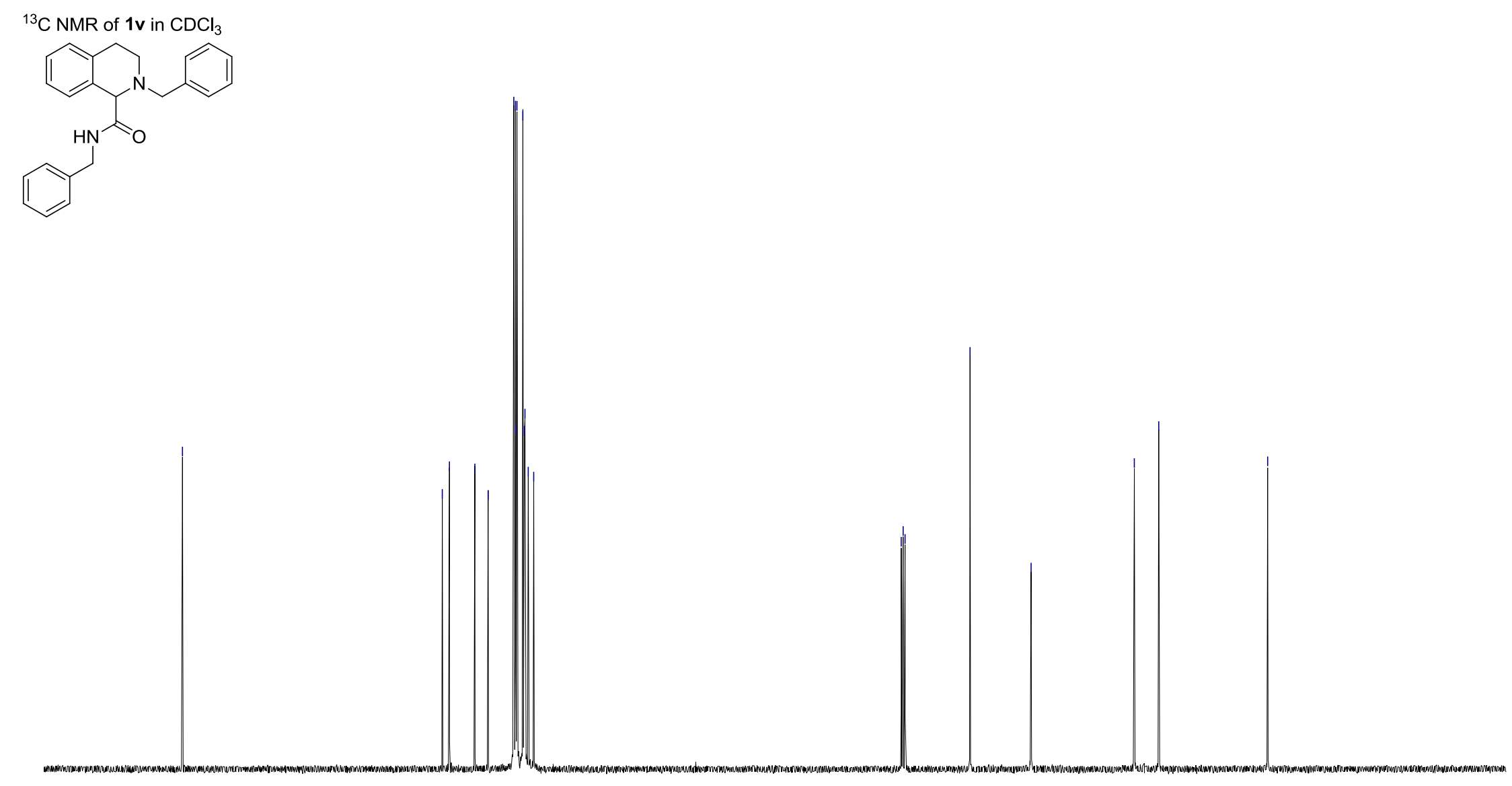

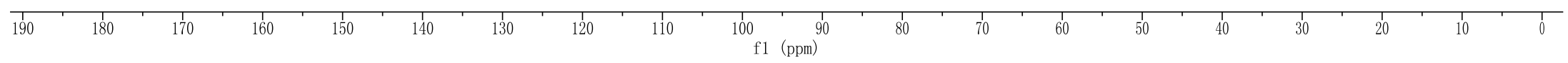




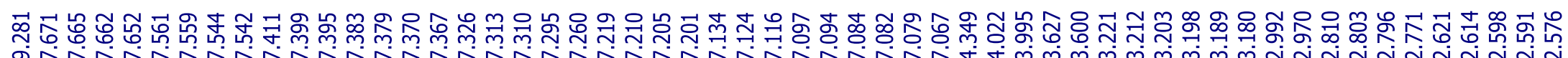

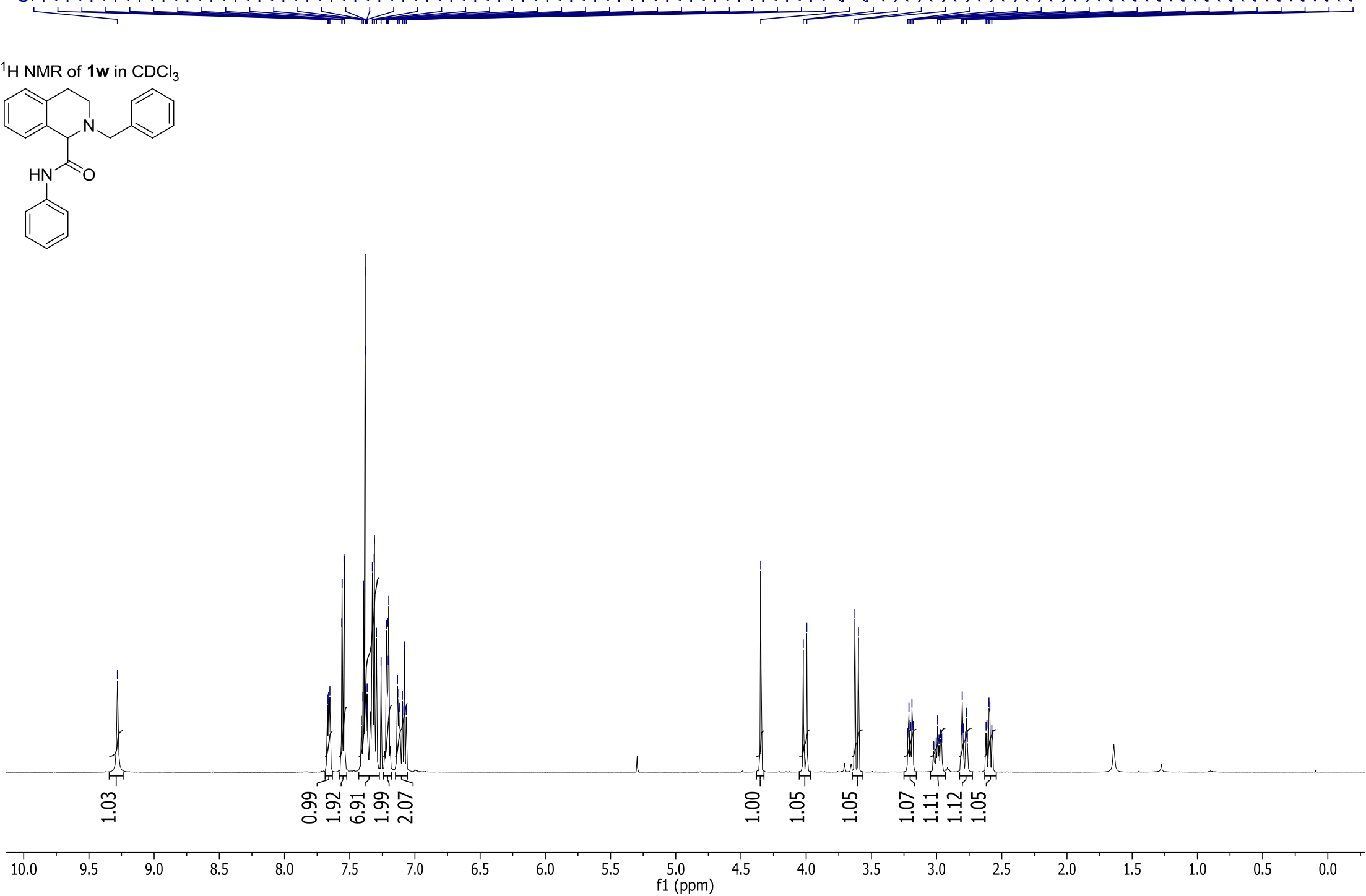




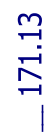

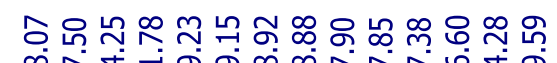

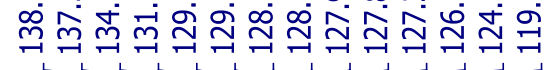

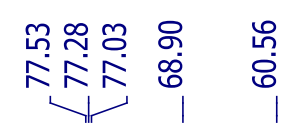
$\underset{\substack{0 \\ \dot{g}}}{1}$
$\stackrel{\substack{\infty \\ \stackrel{\infty}{\sim}}}{\text { | }}$

${ }^{13} \mathrm{C} \mathrm{NMR}$ of $1 \mathrm{w}$ in $\mathrm{CDCl}_{3}$

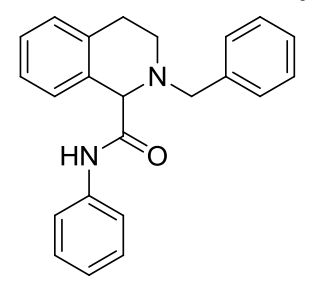

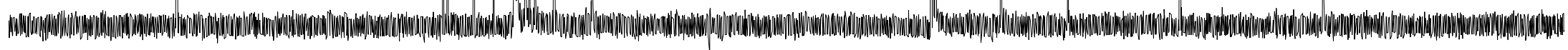

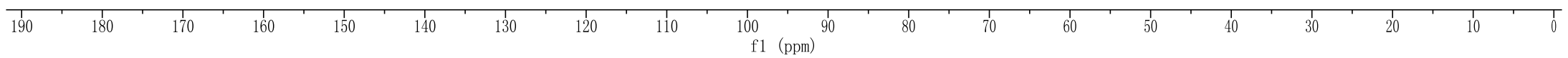




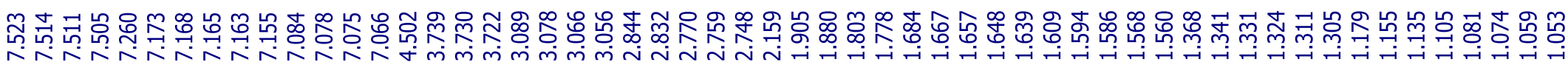

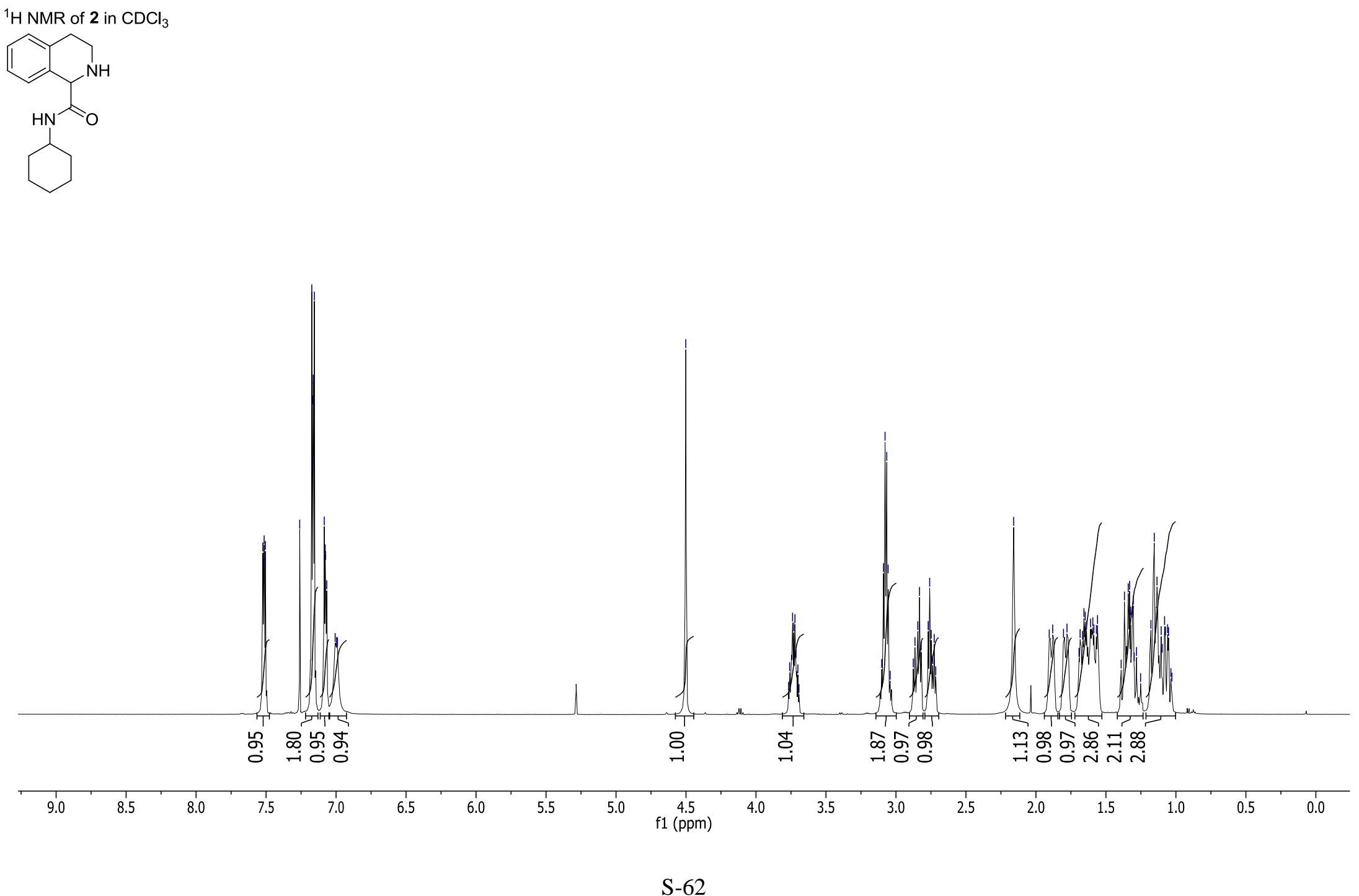




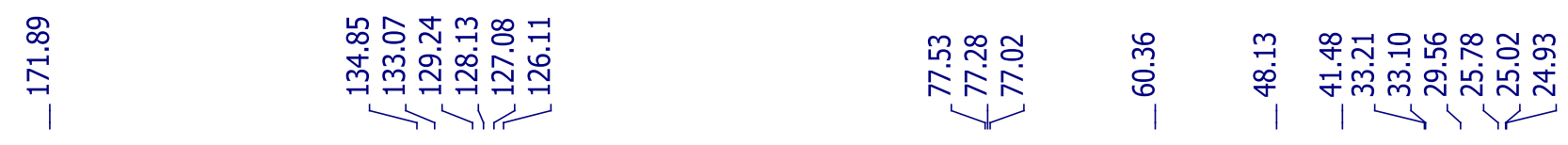

${ }^{13} \mathrm{C}$ NMR of 2 in $\mathrm{CDCl}_{3}$

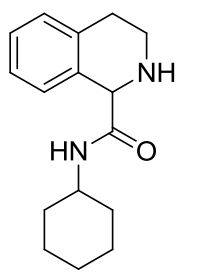

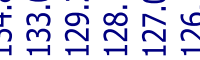

计们

NNN

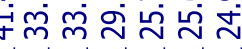




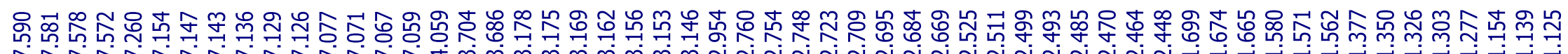

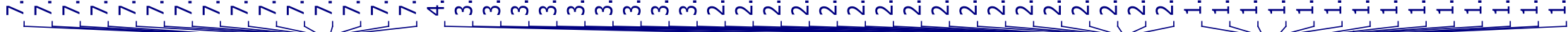
${ }^{1} \mathrm{HNMR}$ of 3 in $\mathrm{CDCl}_{3}$
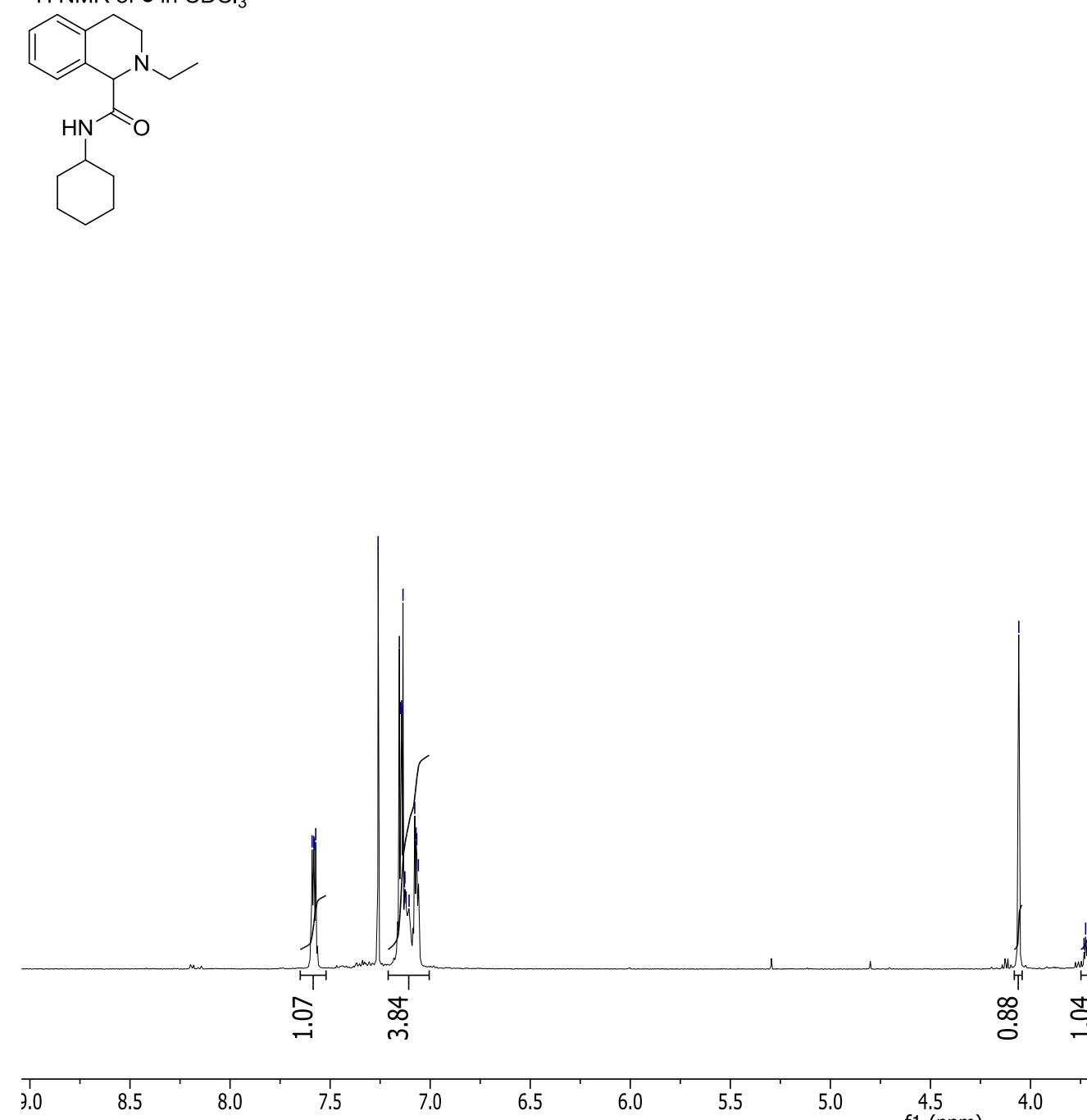

5.5

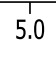

f1 (ppm)

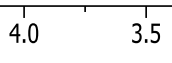




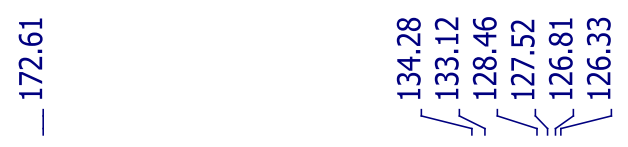

3 in CDR of

政

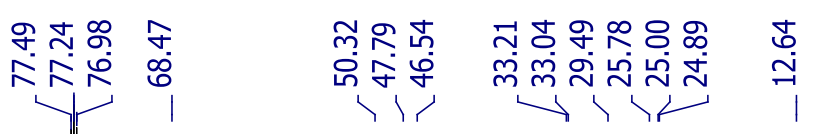

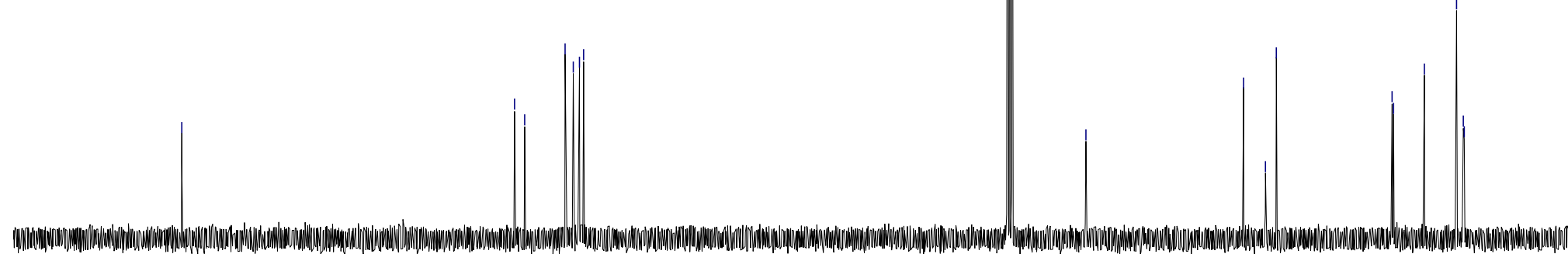

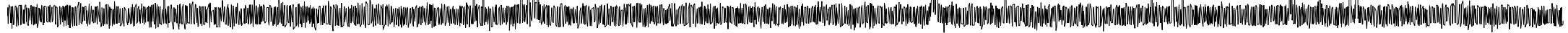

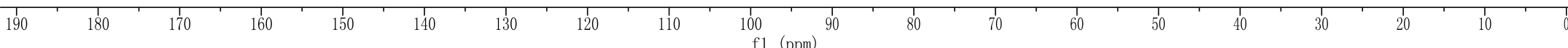




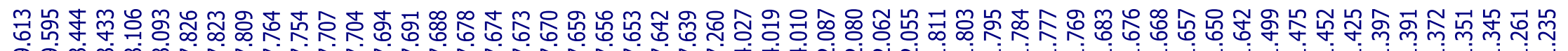
बí

${ }^{1} \mathrm{H}$ NMR of 4 in $\mathrm{CDCl}_{3}$

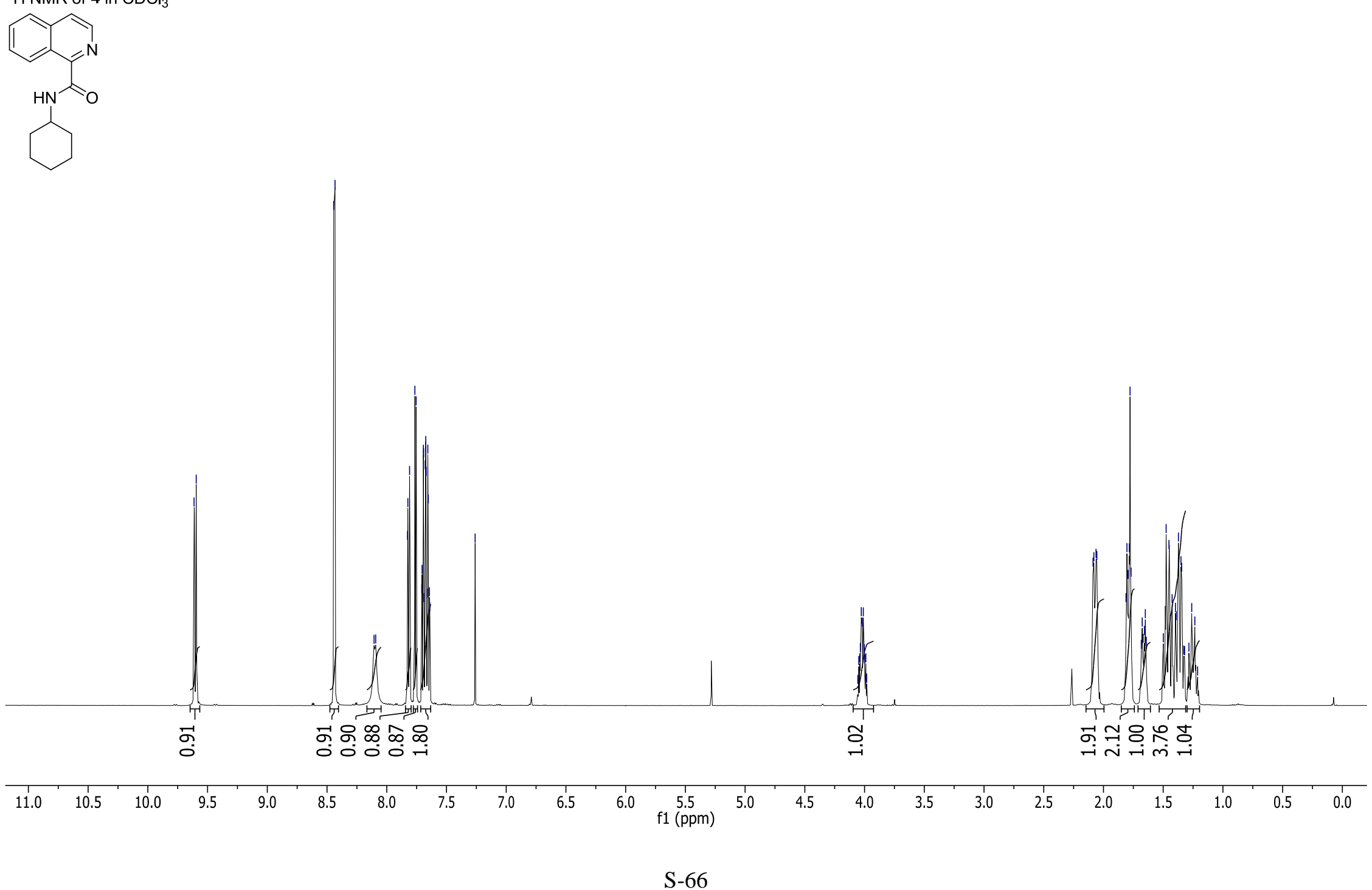




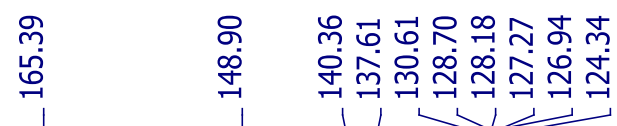

${ }^{13} \mathrm{C} \mathrm{NMR}$ of 4 in $\mathrm{CDCl}_{3}$

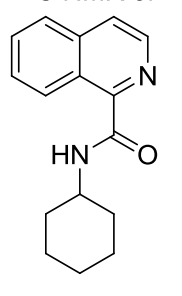

กุกิ จิ

손

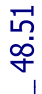

요

m

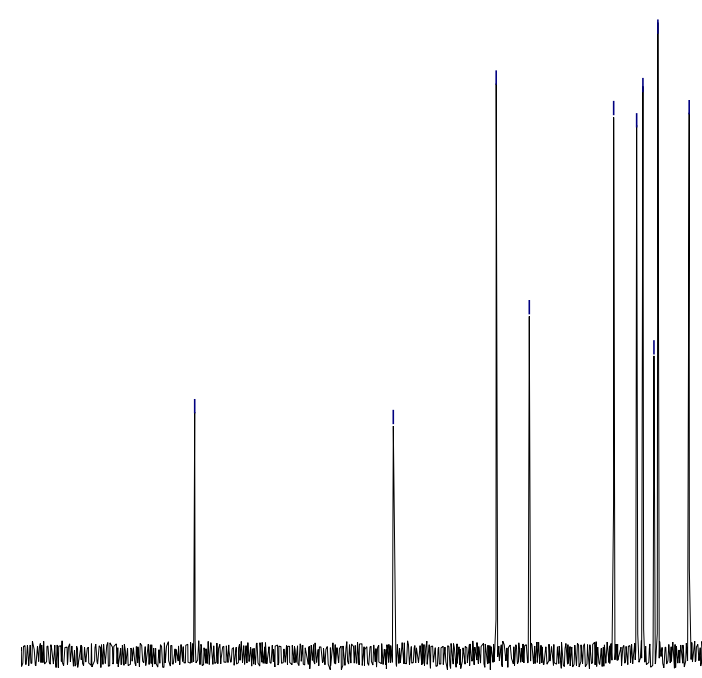

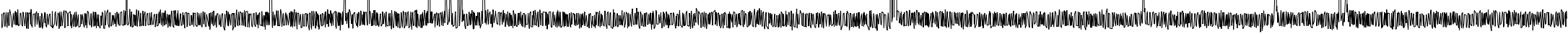

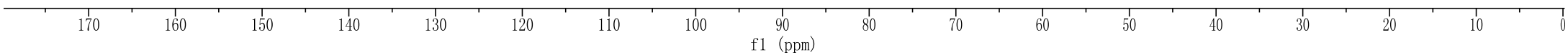


GCOSY of $1 \mathbf{u}$ in $\mathrm{CDCl}_{3}$

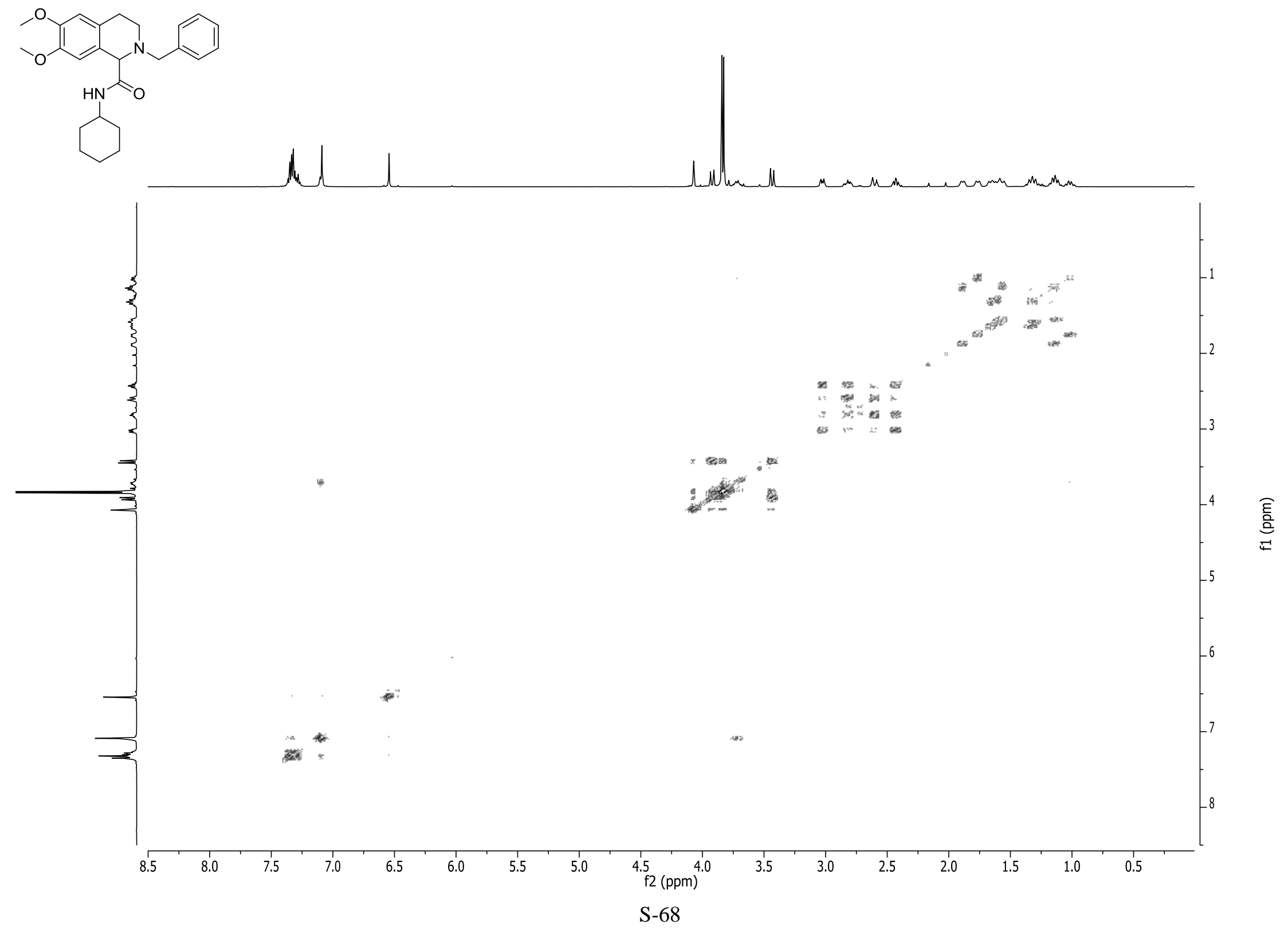




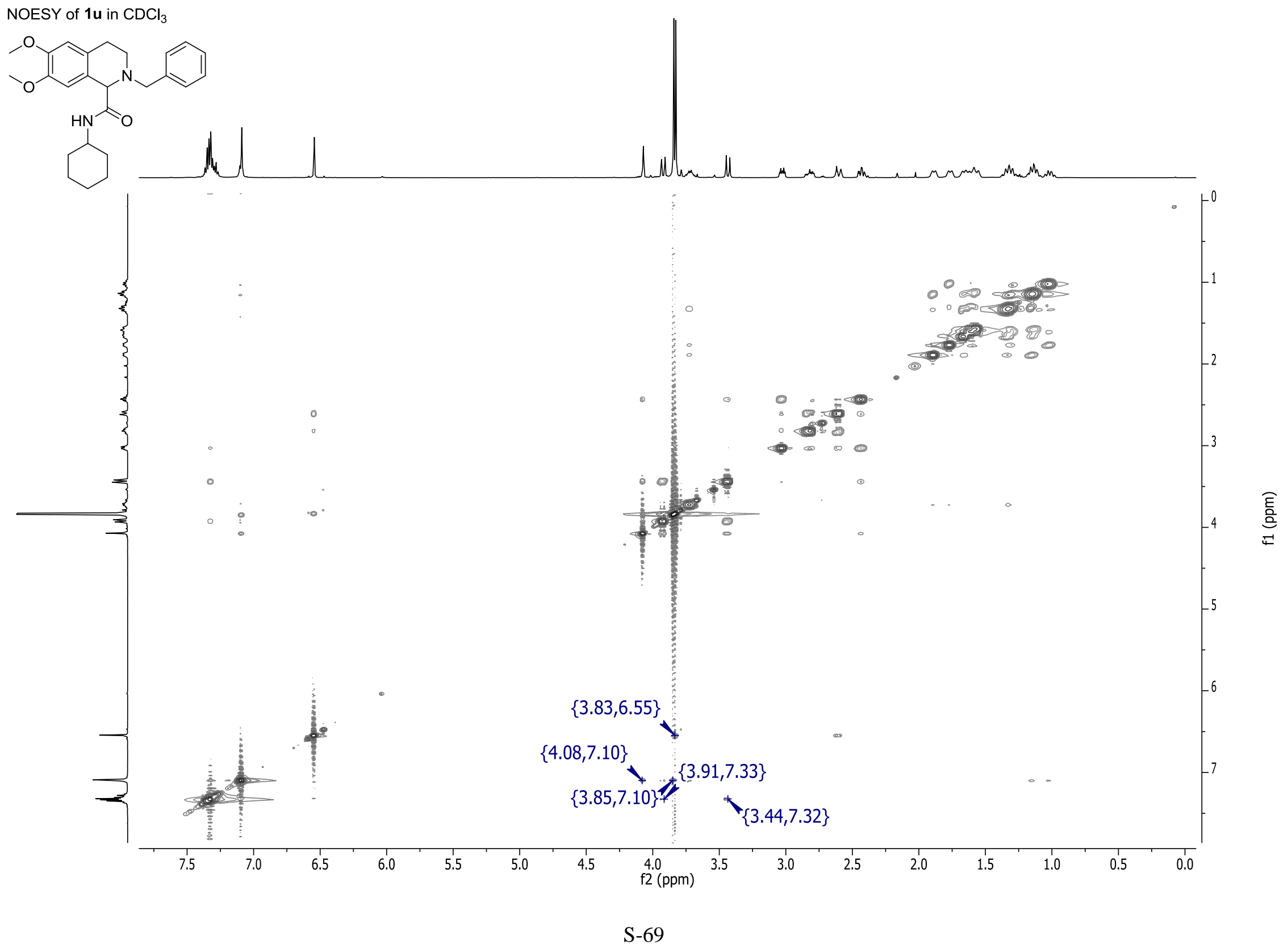

